\title{
WestVirginiaUniversity
}

THE RESEARCH REPOSITORY @ WVU

Graduate Theses, Dissertations, and Problem Reports

2021

\section{Exploring early signaling associated with complex stressors}

Julia A. Penatzer

West Virginia University, jamouch@mix.wvu.edu

Follow this and additional works at: https://researchrepository.wvu.edu/etd

Part of the Pharmacology, Toxicology and Environmental Health Commons

\section{Recommended Citation}

Penatzer, Julia A., "Exploring early signaling associated with complex stressors" (2021). Graduate Theses, Dissertations, and Problem Reports. 8299.

https://researchrepository.wvu.edu/etd/8299

This Dissertation is protected by copyright and/or related rights. It has been brought to you by the The Research Repository @ WVU with permission from the rights-holder(s). You are free to use this Dissertation in any way that is permitted by the copyright and related rights legislation that applies to your use. For other uses you must obtain permission from the rights-holder(s) directly, unless additional rights are indicated by a Creative Commons license in the record and/ or on the work itself. This Dissertation has been accepted for inclusion in WVU Graduate Theses, Dissertations, and Problem Reports collection by an authorized administrator of The Research Repository @ WVU.

For more information, please contact researchrepository@mail.wvu.edu. 


\title{
Exploring early signaling associated with complex stressors
}

\author{
Julia A. Penatzer
}

Dissertation submitted to the Eberly College of Arts and Sciences at West Virginia University

in partial fulfillment of the requirements for the degree of

Doctor of Philosophy in

Chemistry

\author{
Jonathan W. Boyd, Ph.D., Chair \\ Peng Li, Ph.D. \\ John B. Mertz, Ph.D. \\ Stephen J. Valentine, Ph.D. \\ Matthew J. Dietz, M.D. \\ C. Eugene Bennett Department of Chemistry \\ Morgantown, West Virginia \\ 2021
}

Keywords: Stress, Cytokines, Phosphoprotein Signaling, Zephyr, Organophosphate, Corticosterone

Copyright 2021 Julia A. Penatzer 


\title{
ABSTRACT \\ Exploring early signaling associated with complex stressors
}

\author{
Julia A. Penatzer
}

Humans are constantly exposed to a vast number of stressors in our everyday lives from social interactions to physical activity to chemical exposures, to name a few. These stressors have significant impacts on an individual, from the cellular to whole organismal level. The body contains an intricate communication system that elicits a number of both biological and physiological responses as it attempts to maintain homeostasis. Observing these responses to stressors, can offer insight into a number of outcomes, which can be beneficial (increasing life longevity) or detrimental (leading to a disease state). By measuring specific biological adaptations at particular time points, we can better understand the organismal response to these stressors. While human studies provide a unique opportunity to truly understand the organismal response, there are a number of factors that influence individual responses. Therefore, replicating these stressors in vivo can be exceptionally difficult, especially when the primary focus of many studies is on a single stressor, rather than a more realistic combinatory exposure. The work presented in this dissertation had the ultimate goal of building upon previous research with a focus on the biochemical adaptations that take place to protect and maintain optimal organismal function. Specifically, this dissertation observes both extracellular (cytokine) and intracellular (phosphorylation modifications) responses to social, physical, and occupational stressors. Additionally, this work observes both systemic and localized responses in different biological samples (saliva vs ex vivo tissue) to analyze various biomarkers of stress. The findings from this dissertation reveal the rapid responses of signaling mechanisms related to complex mixtures in both human and animal work. 


\section{DEDICATION}

This dissertation is dedicated to my family, Jesse and Aiden Penatzer as well as my parents,

Ed and Beth Mouch, for their unconditional love and support throughout all of my career. 


\section{ACKNOWLEDGEMENTS}

I would first like to thank my advisor and mentor, Dr. Jonathan Boyd, for all his guidance over the last five years. You provided me with numerous scientific opportunities including scientific presentations, in-depth statistical analysis, and manuscript, book, and grant writing, as well as both human and animal work, which have all helped me become a better researcher. I was extremely lucky to have you as an advisor as you shaped me into the person I am today. Thank you for your continuous support and wisdom.

I would like to thank all of my committee members, Dr. Stephen J. Valentine, Dr. Peng Li, Dr. John B. Mertz, and Dr. Matthew Dietz, for their guidance, support, and mentorship during my graduate career. Also, a big thank you to Suzanne Danley who helped make our transition to Orthopaedics as smooth as possible. Thank you, Suzanne, for dealing with more problems than you had to and always lending an ear when I needed you.

To my former lab mates, Dr. Alice Han and Maren Prediger, thank you both for teaching me the ropes in my first years of graduate school. Although we did not physically work together for long, I made two great friends! Alice, you were the best role model and literally taught me how to be a graduate student, but also left me with so many great memories not related to school. Maren thank you for being a friend I could turn to and talk about anything at any time. Also, thank you to Dr. Julie Miller for all your help during my career. Even though we did not both work for Dr. Boyd at the same time, I cannot thank you enough for all your help with NIOSH and editing manuscripts.

A special thank you to all my friends at WVU who helped keep me sane throughout my graduate career. Thank you for being such great friends! From bubble tea companions to game night friends, I am lucky to have gotten to know all of you. I wish everyone the best and hope we 
all stay in touch. I would especially like to thank the honorary Boyd group members, Zach Sasiene, James Lee, and Rob Crovak, for all your help in collecting more saliva than I ever thought possible!

I would especially like to thank Dr. Nicole Prince. Nicole I could literally write a novel to thank you for everything. I was exceptionally lucky to have you as my fellow lab mate throughout our time in Dr. Boyd's lab. From the ups to the downs and everything in between you were always there for me. Thank you for always being someone I could talk or complain or gossip to. Thank you for always being my lunch and Starbucks date when I needed a break. I literally do not think I could have made it without you and I am so happy to have such an amazing friend in my life.

Thank you to my parents, Ed and Beth, my brothers Ryan and Brad, and my sister-in-law Chelsea Mouch. Also thank you to my father-in-law James Penatzer and sister-in-law's Cassidy and Lexi Penatzer. I am grateful for everyone's constant love and encouragement. Even though no one ever knows what I am talking about, it is nice to have you all to talk to and support me.

Finally, I would like to thank my best friend and biggest supporter Jesse Penatzer. My graduate career has been an emotional roller coaster, but I am happy that I have had you along for the ride. Thank you for always listening and giving me advice to the best of your abilities. You have been my rock when I thought I was not good enough. Most of all you have given me all of your unconditional love and I am so happy to have you for the rest of my life. I cannot wait to see where our next adventure takes us. 


\section{TABLE OF CONTENTS}

$\begin{array}{ll}\text { Title Page } & \text { i }\end{array}$

$\begin{array}{ll}\text { Abstract } & \text { ii }\end{array}$

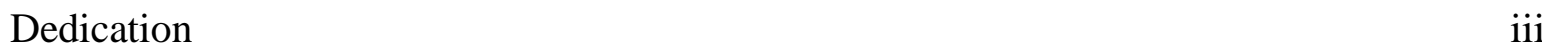

Acknowledgements $\quad$ iv

Table of Contents

List of Figures $\quad$ xii

List of Tables $\quad$ xiii

List of Abbreviations $\quad$ xiv

Chapter 1: Introduction to stress and stressors and the measurable biological and

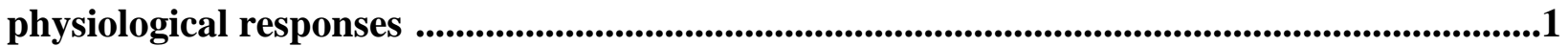

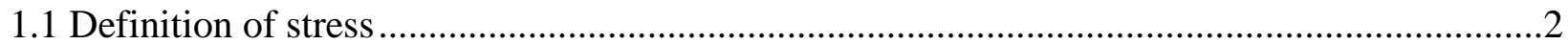

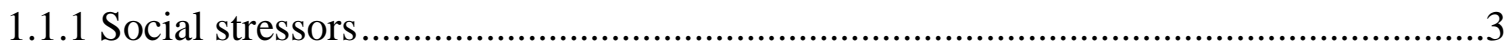

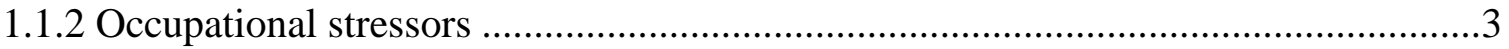

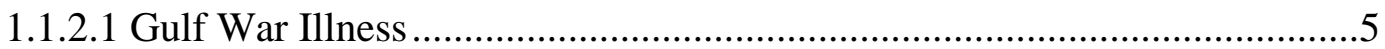

1.2 The biological and physiological responses to stressors...............................................

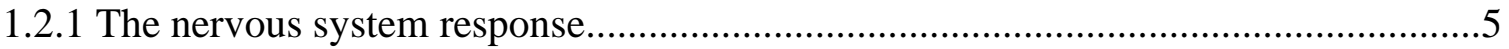

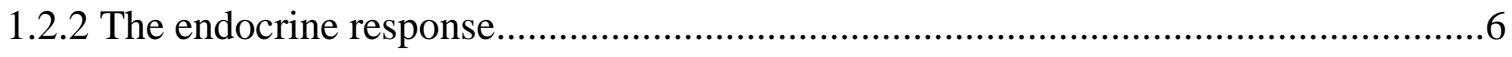

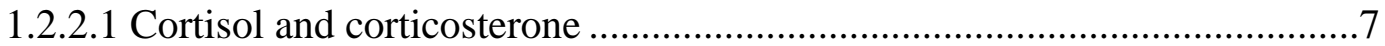

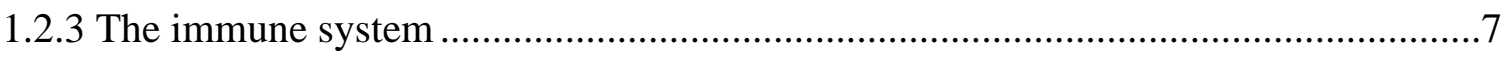

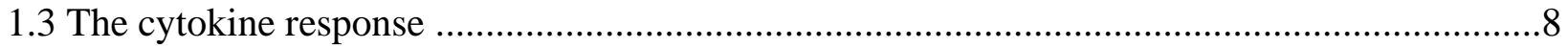




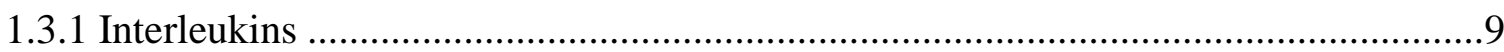

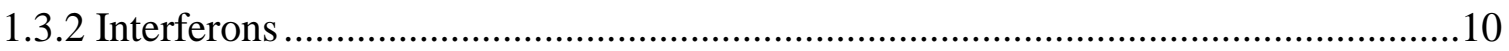

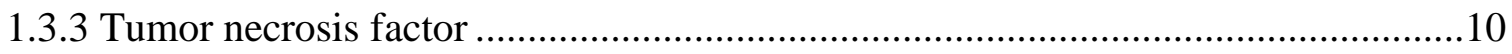

1.3.4 Colony-stimulating factors..................................................................................10

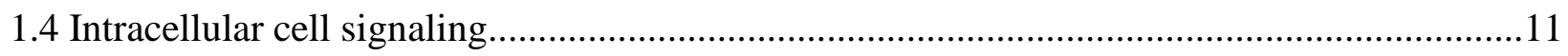

1.4.1 G-protein coupled receptors (GPCRs) …………................................................11

1.4.2 Secondary messangers-cAMP ………………….............................................12

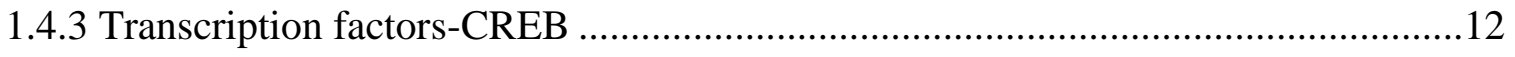

1.4.4 Transcription and translation ..........................................................................12

1.4.5 Post-translational modifications........................................................................13

1.4.5.1 Mitogen-activated protein kinases (MAPKs) ...........................................13

1.4.5.2 Phosphatidylinositol 3-kinase (PI3K)/protein kinase B (AKT) .................14

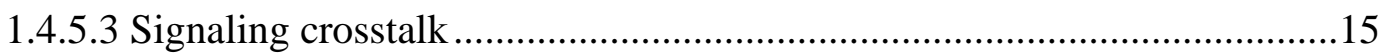

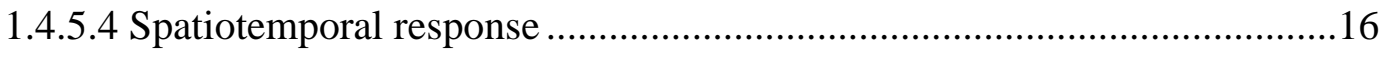

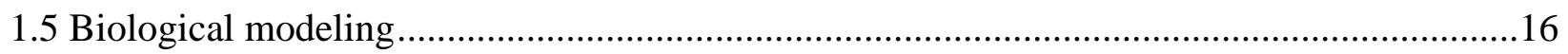

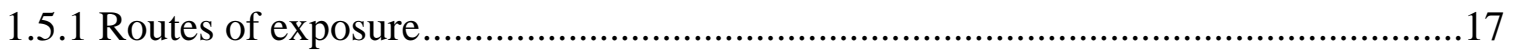

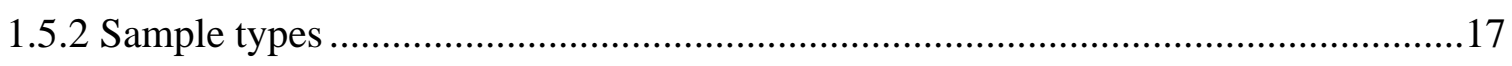

1.5.2.1 Ex vivo tissue ...................................................................................18

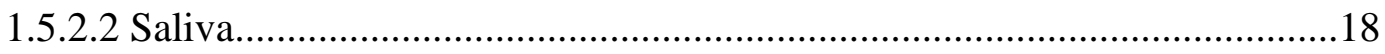

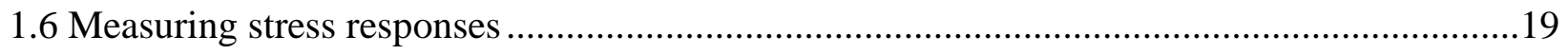

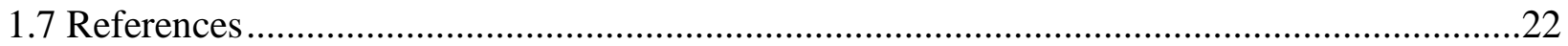




\section{Chapter 2: Salivary cytokines as a biomarker of social stress in a}

mock rescue mission injury

2.1 Introduction .35

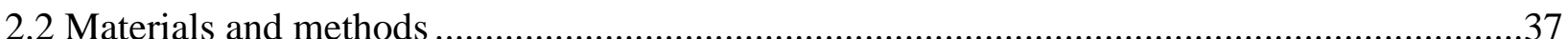

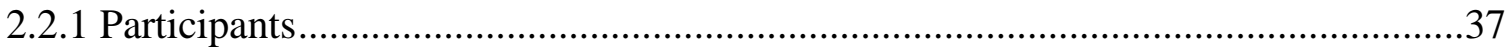

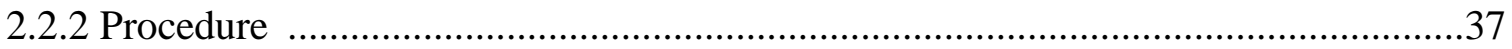

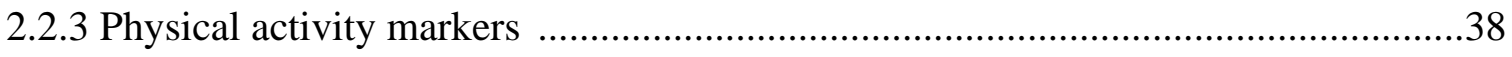

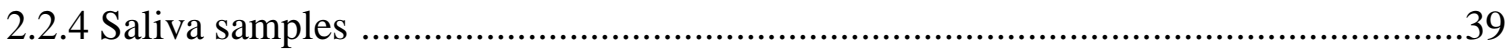

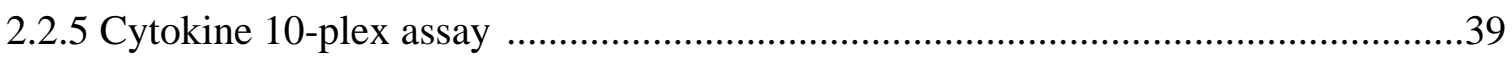

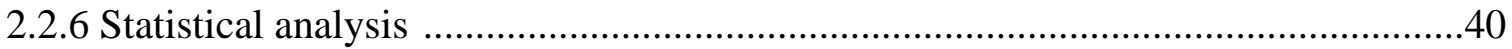

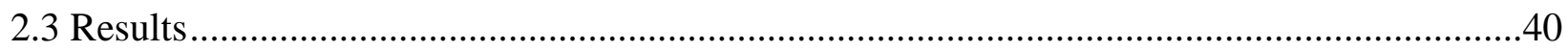

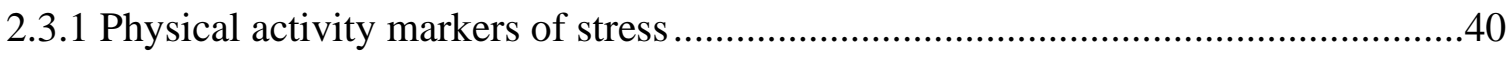

2.3.2 Individual salivary cytokine response to success and failure .............................42

2.3.3 Team salivary cytokine response to success and failure ...................................44

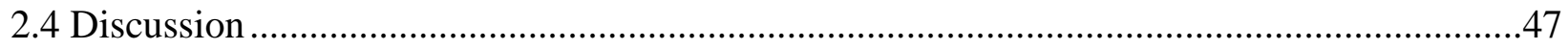

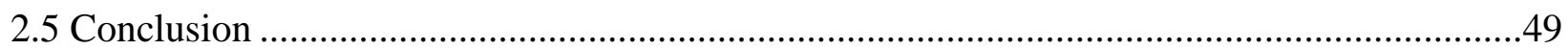

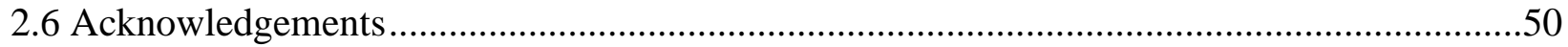

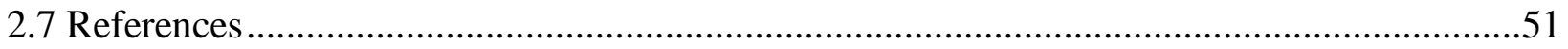

Chapter 3: Corticosterone ameliorates diisopropyl fluorophosphate-induced MAPK phosphoprotein signaling in a mouse brain ...........................................................................................54 


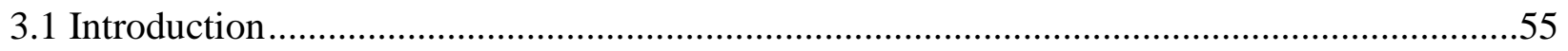

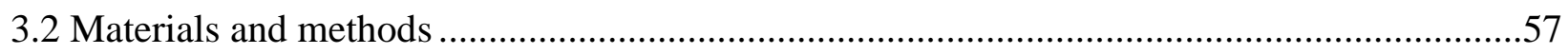

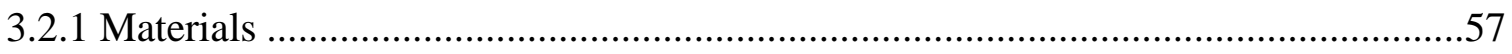

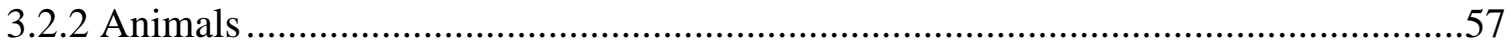

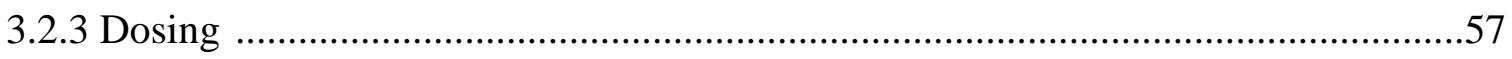

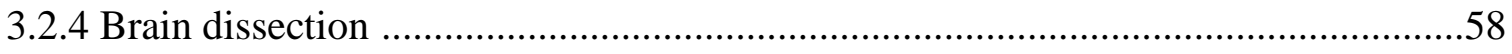

3.2.5 Protein quantification in brain regions .................................................................59

3.2.6 Phosphoprotein analysis in discrete brain regions ...............................................59

3.2.7 Statistical analysis .........................................................................................60

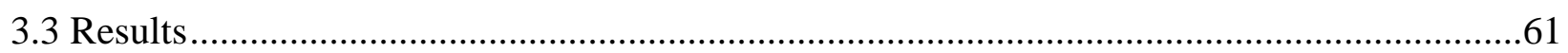

3.3.1 Relative phosphorylation responses in the cortex .....................................................61

3.3.2 Relative phosphorylation responses in the hippocampus ..........................................64

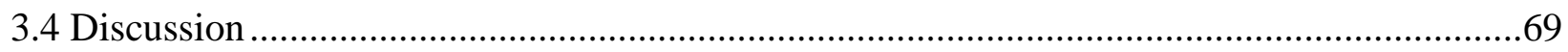

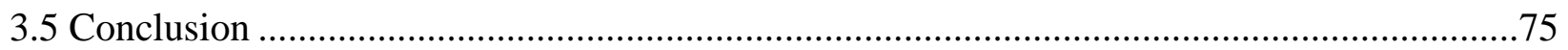

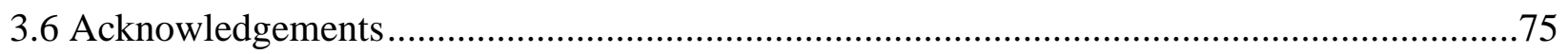

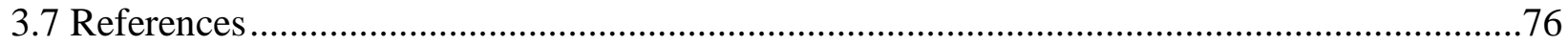

Chapter 4: Corticosterone and chlorpyrifos oxon exposure elicits spatiotemporal MAPK phosphoprotein signaling in a mouse brain ..............................................................................................81

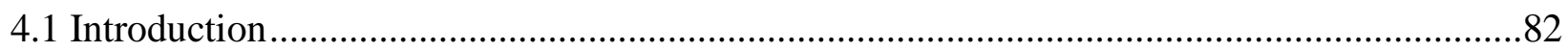

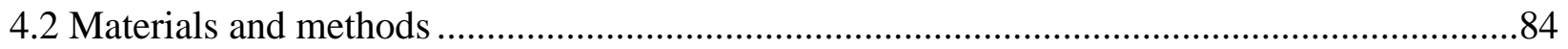

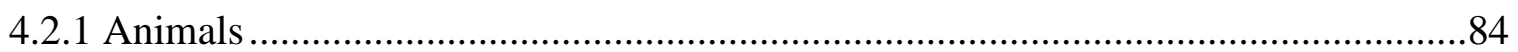




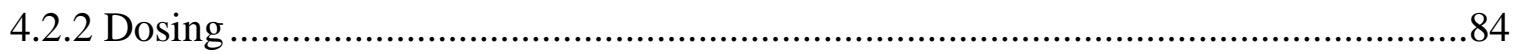

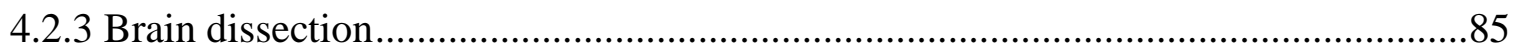

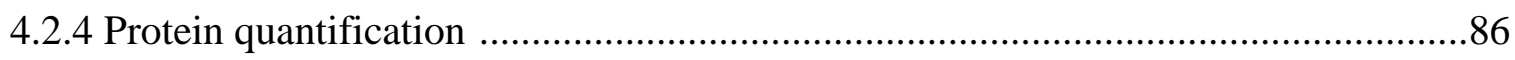

4.2.5 Phosphoprotein analysis in discrete brain regions ....................................................

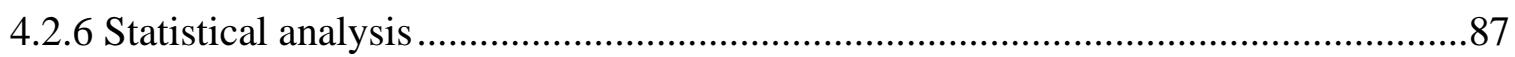

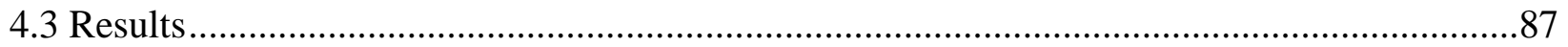

4.3.1 Relative phosphorylation responses in the cortex ....................................................87

4.3.2 Relative phosphorylation responses in the striatum.................................................90

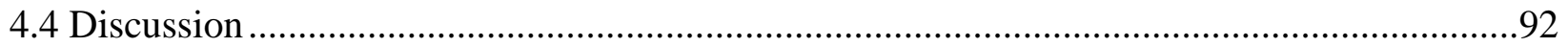

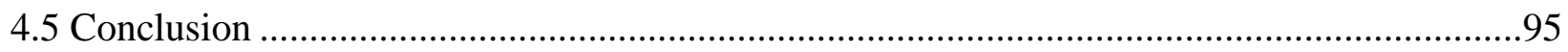

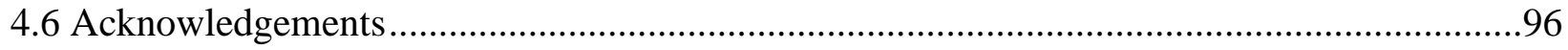

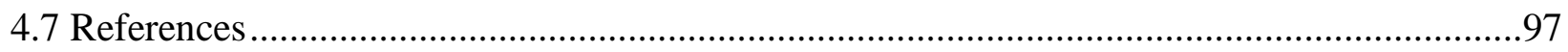

Chapter 5: Differential phosphoprotein signaling in the cortex in mouse models of Gulf War

Illness using corticosterone and acetylcholinesterase inhibitors .................................................102

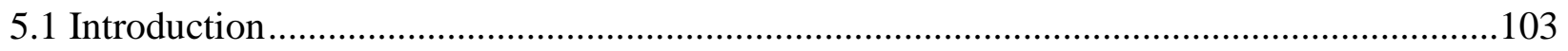

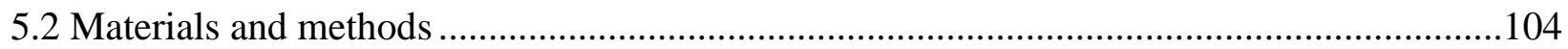

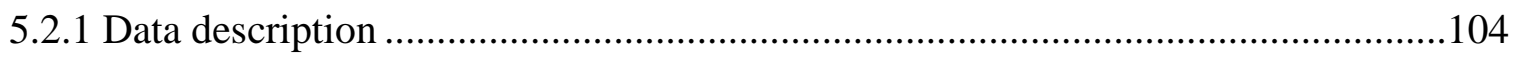

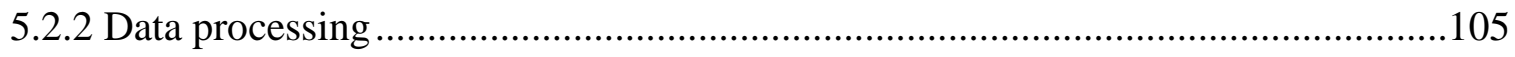

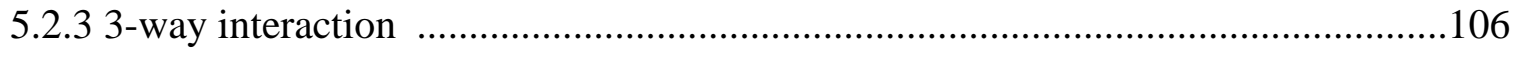

5.2.4 Ingenuity Pathway Analysis (IPA) ...................................................................106

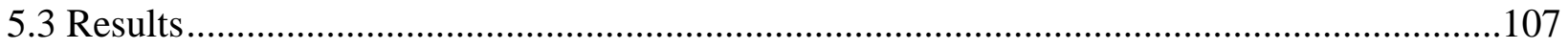




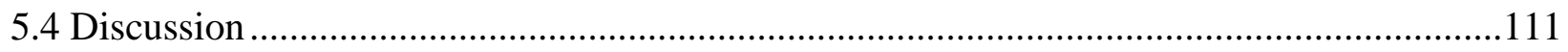

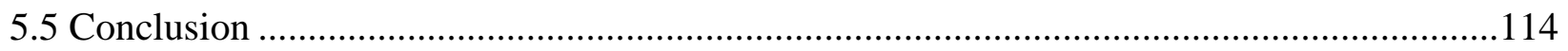

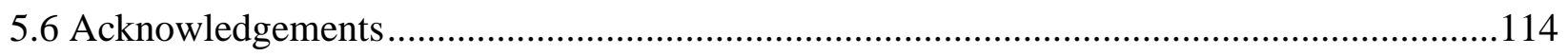

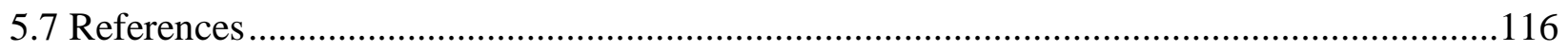

Chapter 6: Conclusions and future directions .......................................................................120

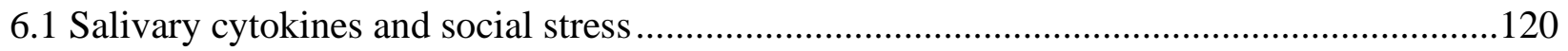

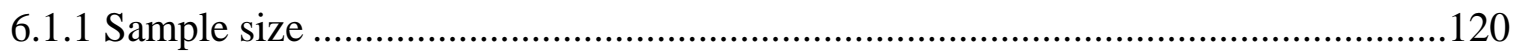

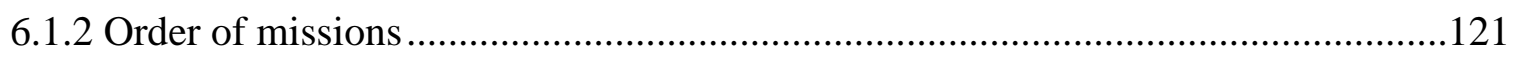

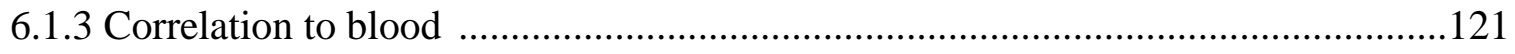

6.2 Phosphoprotein signaling post-corticosterone and organophosphate exposure...................122

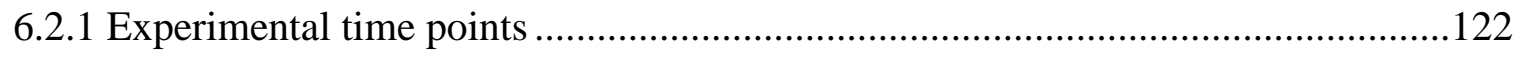

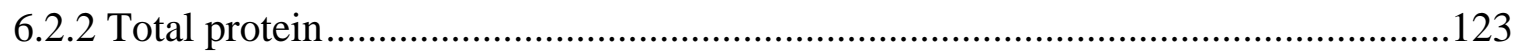

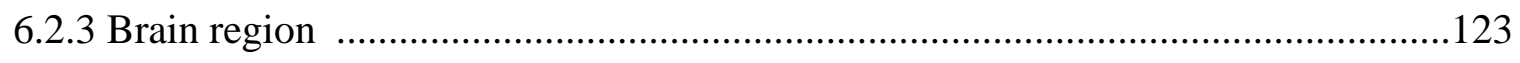

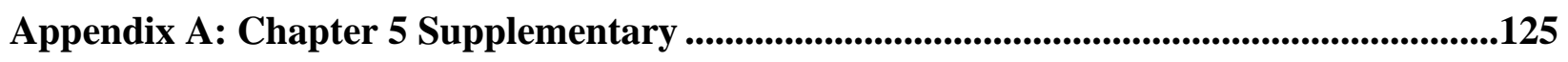




\section{LIST OF FIGURES}

Figure 1.1. Factors influencing the stress response 2

Figure 1.2. Simplified PI3K/MAPK signaling pathways 15

$\begin{array}{ll}\text { Figure 1.3. Bead-based sandwich ELISA } & 20\end{array}$

Figure 2.1 Physical activity markers of stress in a mock rescue mission 41

Figure 2.2 Salivary cytokine concentrations following individual missions 43

Figure 2.3 Salivary cytokine concentrations following team missions 45

Figure 2.4 Cytokine responses pre-mock rescue missions 46

Figure 3.1 Dosing paradigm for corticosterone and diisopropyl 58

fluorophosphate exposure

Figure 3.2 Significant phosphoprotein responses in the cortex via multiplex ELISA 62 post-exposure to corticosterone and diisopropyl fluorophosphate

Figure 3.3. Significant phosphoprotein responses in the hippocampus via multiplex 66 ELISA post-exposure to corticosterone and diisopropyl fluorophosphate

Figure 3.4 Schematic depicting the relationship of SRC with SYK downstream $\quad 74$ PI3K and MAPK signaling

Figure 4.1 Dosing paradigm for corticosterone and chlorpyrifos oxon exposure $\quad 85$

Figure 4.2 Significant phosphoprotein responses in the cortex via multiplex ELISA 89 post-exposure to corticosterone and chlorpyrifos oxon

Figure 4.3 Significant phosphoprotein responses in the striatum via multiplex ELISA 91 post-exposure to corticosterone and chlorpyrifos oxon

Figure 4.4 MAPK signaling pathway following corticosterone with 93 chlorpyrifos oxon exposure

Figure 5.1 Cube plots for p-SRC (Y416) and p-JNK (T183/Y185) 110 


\section{LIST OF TABLES}

Table 2.1 Summary of subjects from mock crime scene house

Table 3.1 Significant temporal phosphoprotein responses in the cortex post-exposure to corticosterone and diisopropyl fluorophosphate

Table 3.2 Significant temporal phosphoprotein responses in the hippocampus post-exposure to corticosterone and diisopropyl fluorophosphate

Table 5.1 3-way interaction revealed distinct phosphoprotein responses in mouse models of Gulf War Illness

Table 5.2 IPA projected nodes

Table A.1 Proposed signaling nodes for a network generated by IPA at $0.5 \mathrm{~h}$ using the experimental dataset

Table A.1 Proposed signaling nodes for a network generated by IPA at $2 \mathrm{~h}$ using the experimental dataset

Table A.1 Proposed signaling nodes for a network generated by IPA at $24 \mathrm{~h}$ using the experimental dataset 


\section{LIST OF ABBREVIATIONS}

OP - Organophosphate

AChE - Acetylcholinesterase

GWI - Gulf War Illness

GW - Persian Gulf War

SNS - Sympathetic nervous system

HPA - Hypothalamic-pituitary-adrenal

PNS - Parasympathetic nervous system

cAMP - Cyclic adenosine monophosphate

$\mathrm{CRF}$ - Corticotropin-releasing factor

ACTH - Adrenocorticotropic hormone

GC - Glucocorticoid

IL - Interleukin

IFN - Interferon

TNF - Tumor necrosis factor

CSF - Colony stimulating factor

GM-CSF - Granulocyte-macrophage colony-stimulating factor

JAK/STAT - Janus kinases/signal transducers and activators of transcription (JAK/STAT)

$\mathrm{NF}-\kappa \mathrm{B}-$ Nuclear factor $\kappa \mathrm{B}$

JNK - c-Jun N-terminal kinase

GPCR - G-protein coupled receptors

PKA - Protein Kinase A

PTM - Post-translation modification

mRNA - Messenger RNA

MAPK - Mitogen-activated protein kinase

ERK - Extracellular signal-related kinase

MKK - Mitogen-activated protein kinase kinase

PI3K - Phosphatidylinositol 3-kinase

AKT - Protein kinase B

PIP2 - Phosphatidylinositol (4,5)-bisphosphate

PIP3 - Phosphatidylinositol (3,4,5)-trisphosphate

ELISA - Enzyme-linked immunosorbent assay

ROTC - Reserve officers' training corps

IRB - Institutional review board

SEM - Standard error of the mean

BMI - Body mass index

CORT - Corticosterone

DFP - Diisopropyl fluorophosphate

I.P. - Intraperitoneal

ANOVA - Analysis of variance

$\mathrm{CPF}$ - Chlorpyrifos

$\mathrm{CPO}$ - Chlorpyrifos oxon

AChEI - Acetylcholinesterase inhibitors

IPA - Ingenuity Pathway Analysis 


\section{Chapter 1: Introduction to stress and stressors and the measurable biological and physiological responses}

Humans are constantly exposed to stressors, including biological, chemical, and physiological stimuli as well as mental and social situations on a daily basis. Stressors broadly represent a "threat" to organismal homeostasis, which initiates a complex stress response within the body that is integral to adapting to the stressor [1]. This response relies on communication between the brain and body through the nervous, endocrine, and immune systems [2-3], which release biochemical messengers to promote adaptation [4]. The regulation of these biological responses occurs through a number of feedback loops to maintain homeostasis, where positive feedback generally refers to the amplification of the response and negative feedback inhibits the response.

It is important to note all of the potential factors that influence the organismal response. Both internal stressors, specifically those that originate from the organism, including genetics, nutrition, and lifestyle choices, and external stressors, stimulants imposed on the organism, encompassing stressors such as occupational exposures, air pollution, socioeconomic status, and social relationships, can impact how an individual will respond to the stressor. Additionally, as commonly termed in toxicology with any exposure, "it is the dose that makes the poison", wherein the duration, frequency, and degree of the stressors will affect the overall organismal response. 


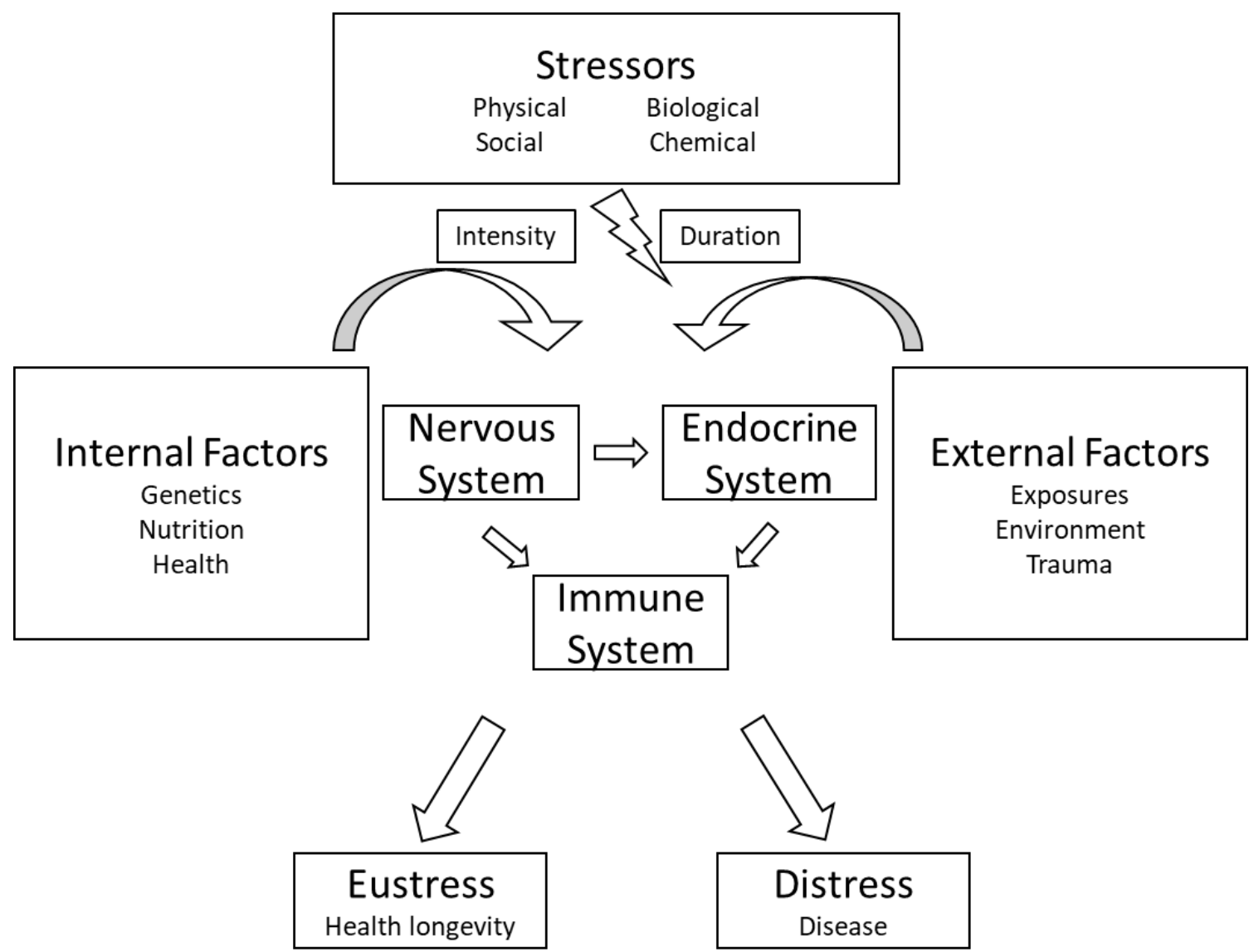

Figure 1.1. Factors influencing the response to stressors. Various stressors (physical, social, chemical) activate the nervous, endocrine, and immune systems to initiate a biological response. This response is based on the intensity and duration of the exposure. Both internal and external factors influence how an individual will respond to the stressors. Stress responses can be either positive or negative.

\subsection{Definition of stress}

In 1936, Hans Selye, the father of modern stress research, first wrote about the biological formulations of stress [6]; stress was used as a descriptive term to explain "the nonspecific response of the body to any demand" [7-8]. In his work, Selye suggested two terms to explain different types of stress: eustress (positive stress) and distress (negative stress) [9]. However, the modern-day term "stress" has taken on the negative connotation as this version has been the focus of numerous studies. According to Bienertova-Vasku et al. (2020), the term distress is used nearly 80 times more than eustress in scientific literature [10]. While too much stress is certainly 
detrimental and can lead to a variety of disease states, there is a critical point at which stress can produce positive results [11-13]. It is also important to be mindful of hormesis, in which moderate exposures can improve functionality and/or tolerance to more severe stimuli, but high doses result in toxicity. Thus, it is critical to appropriately model realistic exposures (e.g., physical, chemical, environmental, social situations, etc.) to fully understand the organismal response.

\subsubsection{Social stressors}

Humans, as an inherently social species, experience both negative and competitive social interactions daily [14-16]. Social interactions start at birth and continue through the remainder of our lives as these connections are necessary for survival [17]. Basic threats to these interactions (e.g., rejection, isolation, conflict, or loss) signal to our body that one is more vulnerable and may face a greater likelihood of wounding and infection, initiating a biological response [18]. Additional research has expanded upon this displaying how social situations such as public speaking, mental arithmetic, and anticipation, as well as emotional states created by social ties, such as anxiety, anger, and depression further increase the biological response [19-24]. Therefore, as social stress is a critical component of our everyday lives, it is important to observe how social experiences, including those experienced in certain occupations, can influence our biological response.

\subsubsection{Occupational stressors}

Some individuals also experience occupational-related stressors, such as occupational hazards (e.g., exposures to chemical, biological, and physical factors), on a daily basis. These hazards are a common health risk for a variety of professions (e.g., health care workers, construction workers, military, farmers, etc.) that can lead to a range of diseases, including chronic obstructive pulmonary disease, cardiovascular disease, and cancer [25-26]. In fact, approximately 
one quarter of all deaths globally are related to working in unhealthy environments with chemical hazards being the leading causative risk factor [27-28].

One particular occupation that is at an increased risk to a number of stressors is the military. Both external exposures (i.e., chemical, biological exposures) and internal, cognitive-based stressors (i.e., traumatic events, information overload) are intricately intertwined into stressors that the military personnel are at risk too while deployed [29]. More importantly, military personnel are often exposed to high dose, acute expedient exposures, while most other occupations would consider this high risk and are more often only exposed to low doses [30].

Organophosphates (OPs) represent one such category of chemical exposures that military personnel can be exposed to during deployment because of the risk of its use as a pesticide and chemical warfare agent [31]. OPs have a similar structure and thus mechanism of action; they bind to acetylcholinesterase (AChE) at the active serine site, which results in cholinergic toxicity due to the persistent stimulation of the cholinergic receptors [32]. Mild exposures can result in headaches, blurry vision, salivation, and muscle weakness, but continued high-level exposure can lead to difficulty breathing, convulsions, irregular heartbeats, and eventually coma or death [33]. Each year there is an estimated 3 million people exposed to OPs worldwide which results in approximately 300,000 deaths [34]. Individuals who survive acute high-level exposure to OPs have extended symptomology associated with eye fatigue, blurred vision, body fatigue, headaches/dizziness, and cognition problems (forgetfulness) [35-36]. In fact, exposure to OP insecticide and nerve agent-OPs have been implicated in a specific military disease state, Gulf War Illness (GWI). 


\subsubsection{Gulf War Illness}

Nearly 200,000 soldiers who served during the 1990-91 Persian Gulf War (GW) returned home from deployment with a variety of chronic, unexplained symptoms that was eventually referred to as GWI [37]. Today, these GW veterans continue to suffer from a multitude of medical symptoms, including cognitive and memory impairments, depression, and gastrointestinal disorders [38-41]. The exact etiology of GWI remains unknown, but the Research Advisory Committee on Gulf War Veterans' Illnesses concluded that exposure to AChE inhibitors, specifically OPs such as sarin [42] or pesticides were the most likely cause of the symptoms of GWI [43-44].

\subsection{The biological and physiological responses to stressors}

In an effort to better understand the localized and systemic responses to these various stressors (e.g., social and occupational stressors), it is important to understand the fundamentals driving the basic biological responses. Once an exposure occurs, the "fight or flight response" is initiated, which regulates the simultaneous activation of a number of biological and physiological. The most heavily studied stress-related systems are the sympathetic nervous system (SNS) and the hypothalamic-pituitary-adrenal (HPA) axis for the nervous and endocrine systems, respectively [45]. Once activated by a stressor, the SNS and HPA axis work cooperatively to coordinate an appropriate response that can be initiated within seconds, but might last for days to restore homeostasis. A brief overview of the role of these systems will be covered below.

\subsubsection{The nervous system response}

The autonomic nervous system, which regulates the body's involuntary actions (breathing, heart rate, blood flow, digestion, etc.) is divided into the SNS and the parasympathetic nervous system (PNS). The PNS is responsible for activities when the body is at rest (e.g., digestion, 
urination, sexual arousal), while the SNS primarily stimulates the body's fight or flight response. Once activated by a stressor, catecholamines, the amine derivatives of catechol that include epinephrine and norepinephrine, are secreted from the adrenal medulla and sympathetic nerves [46]. The catecholamines are then released into the bloodstream which helps increase blood flow to essential organs, such as the skeletal muscle, brain, and heart, to provide an immediate reaction to a stressor [47]. Binding of the catecholamines to $\beta$-adrenergic receptors on the cell surface increases intracellular cyclic adenosine monophosphate (cAMP) and leads to glycogen breakdown, providing the energy needed to mobilize the body to deal with the immediate adaptive response [45, 48]. However, chronic activation of the SNS suppresses the immune system, causes hypertension, and accelerates the onset of a number of cardiovascular diseases [49-51].

\subsubsection{The endocrine response}

Like the SNS, the HPA axis is also crucial in the organismal response to stressors, but, as a hormonal system, activation does not occur until minutes after stimulation [52]. After activation, neurons in the paraventricular nucleus of the hypothalamus synthesize and release corticotropinreleasing factor (CRF) and arginine vasopressin [53]. Binding of CRF stimulates the anterior pituitary gland to produce adrenocorticotropic hormone (ACTH) and release into the body's circulation [54]. This initiates a cascade of events that culminate in the release of glucocorticoids (GCs) from the adrenal cortex [55]. The main GC synthesized in the adrenal glands is cortisol in humans (and corticosterone in rodents), which inhibits insulin production to mobilize glucose reserves for energy [55-57]. GCs play a prominent role in regulating the magnitude and duration of the HPA axis stimulation through a negative feedback loop; specifically, a rapid feedback mechanism mediates inhibition of CRF and ACTH secretion [58]. 


\subsubsection{Cortisol and corticosterone}

Corticosterone is the primary adrenal corticosteroid in rodents because they lack the adrenocortical zona fasciculata enzyme 17- $\alpha$ hydroxylase [56]. Corticosterone is also found in the bloodstream of primates, but 10-20 folds lower than cortisol [56, 59-61]. The majority of cortisol (and corticosterone) is bound to corticosteroid-binding globulin and only a small fraction is biologically active [62-64]. However, both GCs have low molecular weight ( 362 Da for cortisol and $\sim 346$ Da for corticosterone) and are lipophilic, allowing them to passively diffuse into cells, which makes it feasible to measure free cortisol in blood [64-65]. It is important to note that both GCs follow a circadian rhythm with levels peaking early in the morning and gradually decreasing throughout the day [64-66] Importantly, cortisol and corticosterone play an essential role in suppressing the immune response, specifically T cell responses [63-64].

\subsubsection{The immune system}

The immune system may not typically be perceived as part of the fight or flight response, but stressful situations put an individual at an increased risk for potential injury and therefore infection [67]. As such, studies have observed the effects of various stressors on the immune system by measuring markers of inflammation [22, 68-69]. Inflammation, at the tissue level, can be characterized by redness, swelling, heat, and pain that leads to the activation of the immune response on the cellular level. Inflammation is the immune system's response to stressors and acts by eliminating pathogens and initiating healing [70-71]; however. chronic inflammation increases risk for a number of diseases [72-73]. The immune system has two main responses: the adaptive and innate immune response.

The adaptive immune response involves a slow process because of the specificity of the pathogen, but once this defense system has been stimulated, it can provide long-lasting protection 
[67]. The adaptive immune response is carried out by B cells and T cells that involve the antibody response and the cell-mediated immune response, respectively [74]. In the antibody response, B cells are activated to secrete antibodies to bind to foreign antigens encountered in the bloodstream. This directly inactivates the pathogens by preventing them from binding to receptors on host cells [75]. In the cell-mediated immune response, $T$ cells react against an antigen, but only to those bound to receptors on the surface of a host cell.

The innate immune response consists of a number of cells (e.g., neutrophils, macrophages, eosinophils) that deliver a non-specific defense, attacking a number of pathogens [67]. Moreover, the innate response can provide a defense on a short time scale in response to stressors (minutes to hours). Damage-associated molecular patterns (DAMPs) are released in response to injury which promotes the release of hydrogen peroxide and recruits innate inflammatory cells [75]. When the innate immune cells sense DAMPs, the cells start producing cytokines, which can act on the cells that secreted them, nearby cells, or distant cells to be recruited to the site of injury to either maintain an inflammatory response or restores homeostasis [76].

\subsection{The cytokine response}

Cytokines are a large group of soluble glycoproteins secreted by immune cells that play an essential role in signaling the innate immune response. To date, hundreds of cytokines have been identified and they play a role in nearly every biological process including inflammation, disease pathogenesis, non-specific response to infection, and changes in cognitive functions, to name a few [77]. Several features of cytokines may contribute to the various roles they play in the biochemical response. For example, some cytokines have pleiotropic properties (i.e., one cytokine exerts different types of responses on different cell types), such as interleukin (IL)-6 that influences both the adaptive immune responses as well as inflammatory reactions [78]. Another feature some 
cytokines exhibit is functional redundancy (i.e., many cytokines can induce similar effects) [7980]. For example, IL-4 and IL-13 activate common pathways and are suggested to serve redundant functions in vivo [81]. Cytokines can also have synergistic or antagonistic effects, allowing some cytokines to initiate certain functions while others inhibit it.

Importantly, the cytokine response to stressors involves a delicate balance between proand anti-inflammatory cytokines. Pro-inflammatory cytokines are primarily produced by macrophages and promote inflammation and immunity [76]. Some of the best known proinflammatory cytokines include IL-1, IL-12, interferon (IFN)- $\gamma$, and tumor necrosis factor (TNF)$\alpha$ [82]. Conversely, anti-inflammatory cytokines are produced to regulate the pro-inflammatory cytokine response and are secreted from immune cells [76, 82]. IL-10, IL-4, and IL-13 are a few of the most well-known anti-inflammatory cytokines [81]. While some of these cytokines have been well established into these subgroups, other cytokines, such as IL-6, have both properties under different circumstances [83]. Four cytokine classes will be briefly discussed below: ILs, INFs, TNFs, and colony-stimulating factors (CSFs).

\subsubsection{Interleukins}

ILs play essential roles in activation and differentiation of immune cells and are primarily expressed by leukocytes [84]. They consist of a large group of proteins that have both pro- and anti-inflammatory properties and primarily modulate growth, differentiation, and activation during immune responses. ILs have a mostly localized effect with a short circulation time that is tightly regulated by positive and negative feedback loops to establish this delicate balance between proand anti-inflammation [85]. 


\subsubsection{Interferons}

IFNs are proteins that were originally discovered to defend against viral infections, but have a number of diverse functions including modulating inflammatory responses, inhibiting (or stimulating) cell growth, inhibiting (or activating) apoptosis, and modulating multiple components of the immune system [85]. IFNs can be broken down into two main groups: type 1 and type 2 . Type 1 comprises 13 different IFN- $\alpha$ isomers, IFN- $\beta$, IFN- $\kappa$, IFN- $\omega$, and IFN- $\varepsilon$ and are induced by viral infections [85-86]. Type 2 IFNs, synthesized by T and natural killer cells, consist of only one isomer, IFN- $\gamma$, which plays an essential role in the cell-mediated immune response [87]. IFN signaling has also been linked to the Janus kinases/signal transducers and activators of transcription (JAK/STAT) pathway, which activates a number of downstream signaling cascades [88-89].

\subsubsection{Tumor necrosis factor}

TNF is produced by phagocytes, neutrophils, lymphocytes, endothelial cells, and fibroblasts and contributes to cell survival, proliferation, differentiation, and death [90-91]. TNF is another pleiotropic cytokine that has anticancer properties, regulates the immune system, and protects against infections, but also stimulates proliferation and migration in cancer cells and is linked to several genetic diseases [91-93]. Another example of TNF's pleiotropic role involves the activation of both nuclear factor $\kappa \mathrm{B}(\mathrm{NF}-\kappa \mathrm{B})$ and c-Jun N-terminal kinase (JNK), which function as cell survival and cell death signaling pathways, respectively [91].

\subsubsection{Colony-stimulating factors}

CSFs have been termed the master regulators of granulocytes and macrophages [94]. CSFs have five major actions that include apoptosis suppression, proliferative stimulation, lineage commitment decisions (whether a precursor cell produces granulocytic or macrophage progeny), 
initiation and regulation of maturation, and functional activity of mature granulocytes and macrophages [94-95]. There are four distinct types of CSFs: granulocyte colony-stimulating factor, macrophage colony-stimulating factor, granulocyte-macrophage colony-stimulating factor (GMCSF), and multipotential colony-stimulating factor (IL-3) [96]. CSFs are strictly regulated and have a short half-life in vivo, but have been shown to significantly increase 1,000-fold within hours after stimulation [94].

\subsection{Intracellular cell signaling}

While looking at the immune system provides important insights into the inflammatory response, it is also important to note the cascade of intracellular signaling that can result. This cascade is the primary way the cell responds to the environment when receiving extracellular signals (e.g., hormones, xenobiotics, cytokines, etc.). As previously mentioned, immune responses can activate distinct signaling pathways that can lead to an adaptive stress response that can result in a return to homeostasis, cell death, or adaptation to a new diseased steady state [97].

\subsubsection{G-protein coupled receptors (GPCRs)}

In order for the cell to respond to changes in the environment, the cell surface receptors must be able to receive and process signals. G-protein coupled receptors (GPCRs) are the largest family of membrane proteins and can be divided into five subfamilies: rhodopsin, secretin, glutamate, adhesion, and Frizzled/Taste2 [98-99]. All of the families share a common structural motif, the seven membrane-spanning $\alpha$-helical segments connected to alternating intracellular and extracellular loops [98-99]. Binding of endogenous hormones, neurotransmitters, and pharmacological agents to GPCRs initiate a conformational change that activates secondary messengers [100]. 


\subsubsection{Secondary messengers-cAMP}

Secondary messengers are produced or released through the signals from the receptor that then propagate signals throughout the cell [101]. Secondary messengers mediate a number of biological processes including memory, metabolism, gene regulation, and immune function [102]. cAMP is a common secondary messenger that is synthesized from ATP by adenylyl cyclase. cAMP concentration, duration, and distribution depend on the activity of phosphodiesterase, which degrades cAMP [103]. cAMP then binds to target proteins such as Protein Kinase A (PKA).

\subsubsection{Transcription factors-CREB}

Transcription factors are proteins that function downstream in the signaling cascade and are involved in the process of initiating and regulating the transcription of DNA into RNA. CREB, the best characterized stimulus-induced transcription factor, was originally identified as a target of the cAMP signaling pathway, but is now known to be activated by several kinases including PKA, Protein Kinase C, and p90 ribosomal S6 kinase [104-105]. Activation through all of these pathways leads to a post-translation modification (PTM), phosphorylation, of CREB at the serine 133 residue, which then interacts with CREB-binding protein to initiate transcription of CREBresponsive genes [105]. CREB contains a C-terminus basic domain that binds to DNA target sequences and a leucine zipper domain that controls dimerization, while the remaining domains help facilitate interactions with coactivators to ultimately carryout RNA synthesis [105-106].

\subsubsection{Transcription and translation}

The flow from DNA to protein is transmitted through messenger RNA (mRNA). The main enzyme involved in transcription is RNA polymerase, which synthesizes RNA molecules to form the complementary base pair of the DNA sequence in the form of pre-mRNA. Pre-mRNA must undergo extensive processing, removing introns and splicing together exons, as well as capping 
the $5^{\prime}$ end and polyadenylation at the $3^{\prime}$ end [107]. The mature mRNA is then transported to the cytosol where it is translated into protein by ribosomal RNA using transfer RNA to supply the amino acids to the peptide chain [107].

\subsubsection{Post-translational modifications}

As mentioned with CREB, signaling networks are regulated by the presence of PTMs. PTMs involve the attachment of small chemical moieties to amino acid residues (e.g., phosphorylation, acylation, alkylation, glycosylation) [101]. The most common mechanism to regulate function and transmit signals in cell signaling is phosphorylation [108]. The importance of phosphorylation in cell signaling is demonstrated by the fact that protein kinase domains are found in 1.5-2\% of genes [109-110]. Protein kinases can adopt an "on" or "off" switch following the transfer of $\gamma$-phosphate of ATP to the hydroxyl group of serine, threonine, or tyrosine. The spatial and temporal control of phosphorylation on serine and threonine residues include cell-cycle

progression and cellular growth, while tyrosine phosphorylation controls cellular proliferation and differentiation [111-112].

\subsubsection{Mitogen-activated protein kinases (MAPKs)}

MAPKs have been studied in numerous diseases because of their diverse role in regulating proliferation, differentiation, gene expression, cell survival, and apoptosis. MAPKs can be stimulated by a plethora of stressors and work upstream to phosphorylate diverse substrates that leads to a cascade of signaling events that initiate changes for the appropriate biological response [113]. Overall, MAPKs are grouped into three main families: extracellular signal-regulated kinases (ERKs), c-Jun N-terminal kinases (JNKs), and p38.

The ERK1/2 pathway is the best studied pathway in the MAPK system. Activation of the ERK pathway occurs by various stimuli including signals from cell-surface cytokine receptors 
(e.g., IL-3, GM-CSF) [114-115]. Activation of this pathway has been extensively studied and it is well understood that cell surface receptors activate small GTPase RAS, which recruits RAF for subsequent phosphorylation [116-117]. RAF, in turn, activates MEK1/2, which then phosphorylates ERK1/2. The activation of this cascade regulates proliferation, differentiation, and apoptosis [117-118].

JNKs are activated by environmental stressors (oxidative stress, heat, DNA damage) and inflammatory cytokines. There are three genes (with 12 isoforms) that encode JNK: JNK1 and 2, which are found in nearly every cell and JNK3 that is mainly found in the brain [119-120]. Signaling occurs through the mitogen-activated protein kinase kinase (MKK) 4 and 7, which lead to the phosphorylation of JNK. Generally, JNK is a pro-apoptotic pathway and plays a critical role in apoptosis, angiogenesis, and migration $[119,121]$. Interestingly, this pathway acts as a critical point between the adaptive and innate immune responses because it can be activated by Toll-like receptor ligands as well as proinflammatory cytokines [122].

p38, like JNKs, are activated by environmental stressors (UV irradiation, heat, lipopolysaccharide) and inflammatory cytokines [120]. p38 signaling is activated by MKK3 and MKK6 kinases, but can also be activated by MKK4 $[119,121]$. There are 4 genes that encode p38: $\mathrm{p} 38 \alpha$ and $\mathrm{p} 38 \beta$ that are ubiquitously expressed, and $\mathrm{p} 38 \gamma$ and $\mathrm{p} 38 \delta$ that have more restrictive expression $[119,123]$. p38 plays a tumor suppressor role and can regulate inflammation, apoptosis, differentiation, and cell cycle regulation [113, 119].

\subsubsection{Phosphatidylinositol 3-kinase (PI3K)/protein kinase B (AKT)}

The phosphatidylinositol 3-kinase (PI3K)/protein kinase B (AKT) signaling pathway has also been extensively studied as a hallmark for many different disease states, especially cancer [124-125]. This pathway responds to a number of stimuli, including growth factors and cytokines 
[125-128]. The PI3K/AKT pathway is highly conserved following a tight multi-step process similar to ERK: activation of PI3K converts phosphatidylinositol (4,5)-bisphosphate (PIP2) to phosphatidylinositol (3,4,5)-trisphosphate (PIP3), which then activates AKT [129]. This pathway plays a role in regulating diverse cellular functions such as metabolism, proliferation, survival, and growth [128].

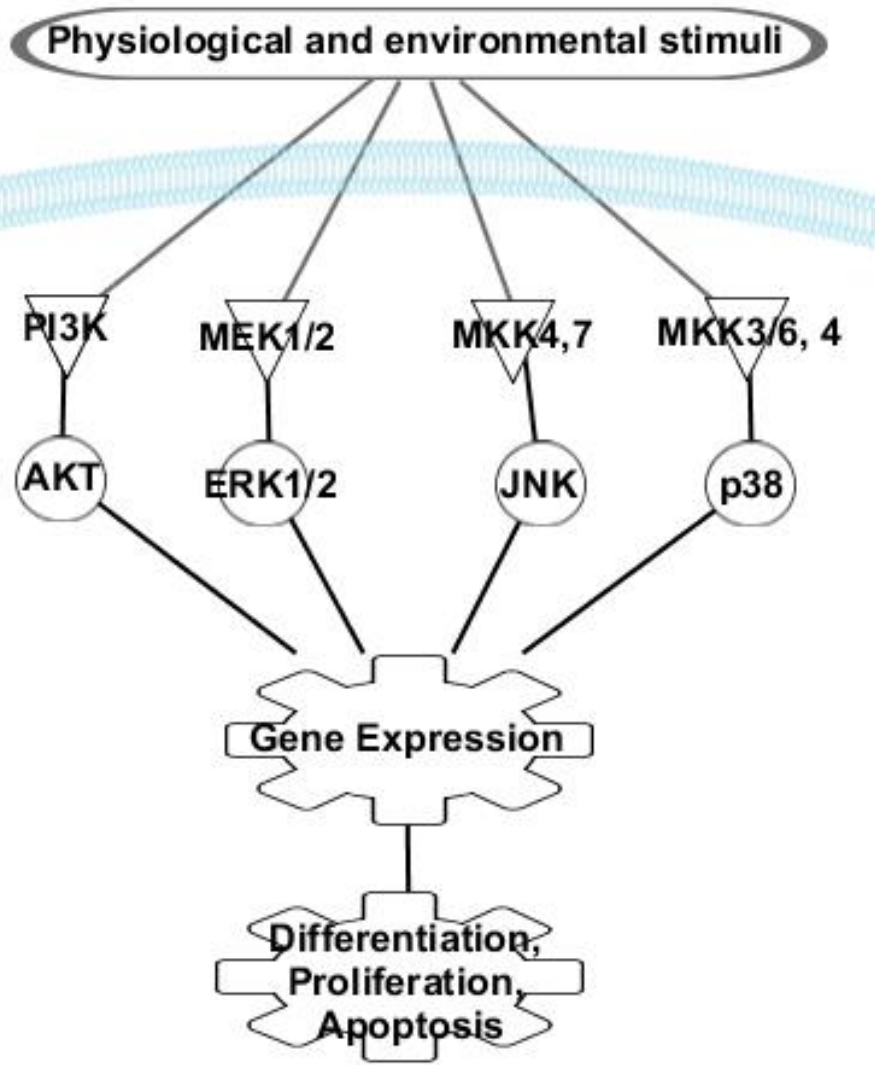

Figure 1.2. Simplified PI3K/MAPK signaling pathways. Physiological and environmental stimuli can phosphorylate a number of signaling pathways including the mitogen-activated protein kinase (MAPK) and the phosphatidylinositol 3-kinase (PI3K) signaling pathways. See text for details.

\subsubsection{Signaling crosstalk}

Importantly, while these pathways have been well studied separately, recent research has focused on the crosstalk between pathways [130-131]. For example, the ERK1/2 and PI3K pathways were originally modeled as linear signaling pathways, activated by different stimuli, but 
research has demonstrated that these pathways can regulate each other and, together, can regulate common downstream functions [132]. This multi-pathway crosstalk can lead to highly extensive networks with significant complexity based on diverse cellular responses to the same stimuli [127, 132]. Thus, after the toxicant exposure, the organism-level response must be organized via spatial (e.g., brain region and/or cell type) and temporal (e.g., acute and adaptive) cellular responses, to provide the appropriate insight into both the short- and long-term implications in response to stressors [101, 133-134].

\subsubsection{Spatiotemporal Response}

The initial cell signaling responses post-exposure to stressors can be monitored within the first few seconds to minutes, but the time course to cell death can have a vast range (several hours to even days) [135-138]. Moreover, analysis of discrete tissue regions allows discrimination of local xenobiotic toxicity that may otherwise go undetected in pooled samples [139]. By observing discrete tissue regions at specific time points, one is able to get a more complete organismal response to these various stressors (e.g., infection, trauma, exposure, etc.) [140-142].

\subsection{Biological modeling}

When investigating the biological response to stressors and attempting to extrapolate studies to human diseases, it is important to choose an appropriate model. While in vitro assays are high throughput, they provide only a simple snapshot of a controlled environment. In vivo studies can provide a comprehensive analysis of a complete biological system. Typically, these include animal models, from rodents to primates that can provide more insight and can be extrapolated to the human population.

Many studies often focus on in vivo exposures to a single xenobiotic, but, for the most part, humans are constantly exposed to a number of complex mixtures on a daily basis, including stress 
[143-145]. In 2015, 24\% of adults in the United States reported extreme levels of stress and roughly $33 \%$ reported significant increases in stress levels from the past year [146]. In 2020, COVID-19 led to an increase to about $60 \%$ of people experiencing increased stress [147]. Thus, to more adequately represent exposures, it is important to address the mixed exposure profile, especially with stress (i.e., cortisol).

\subsubsection{Routes of exposure}

To get the most relevant information to the human population, it is also important to consider the different types of in vivo exposures. The most common dosing methodology include oral, dermal, inhalational, and injection (e.g., subcutaneous, intraperitoneal, intramuscular). While injections might be the least appropriate to "real-life" exposures, it is commonly used because it produces the highest bioavailability of the substance since it avoids first-pass elimination (xenobiotic is metabolized before reaching its site of action) [101, 148-150]. This route circumvents some of the unpredictability of the concentration that is administered.

\subsubsection{Sample types}

Biological samples, such as tissues, blood, urine, and saliva are used in a variety of fields for basic, clinical and epidemiological studies to measure the biological response to stressors [152]. In both human studies and animal models, it is critical to test the appropriate biological sample and acknowledge a systemic versus localized response. For example, in a physical trauma (i.e., broken femur) it may be important to monitor the immediate localized tissue healing response, but a psychological stressor (i.e., a competition) might best be monitored by looking at a systemic response. Despite knowing a target organ for an exposure (e.g., diisopropyl fluorophosphate, an $\mathrm{AChE}$ inhibitor, will target the brain), accessibility to the target tissue is also important, especially in studies involving chronic exposures. In order to monitor an animal over time, systemic 
measurements (blood, saliva, sweat, cerebral spinal fluid) may need to be taken until euthanasia to avoid large numbers of animals. This dissertation will focus on two types of biological sampling: ex vivo tissue and saliva.

\subsubsection{Ex vivo tissue}

Ex vivo tissue sampling can provide the localized spatiotemporal snapshot of the biological response to a stressor. When obtaining tissue samples, it is important to be aware of euthanasia methods and how this can affect the tissue prior to testing. Some common euthanasia techniques, including inhalation of carbon dioxide, decapitation, or cervical dislocation, are used because of their low cost (if any) and ease of use, but can have profound effects on certain analytes of interest, the tissue quality, and data reproducibility [153]. Conversely, a less common technique such as focused microwave irradiation, can preserve the steady-state levels of biological activity as well as maintain tissue quality [154].

Once euthanized, tissue samples can be excised and snapped frozen to mitigate potential gene alterations or protein phosphorylations [155]. It may also be important to further isolate specific cell (e.g., immune cells) or muscle types (e.g., skeletal muscle) for a more focused investigation. However, it is important to acknowledge inconsistencies in tissue samples, such as size of sample, scar tissue, fat, etc., that occur by human error. These differences can yield apparent significant findings that are inconsistent with the true responses.

\subsubsection{Saliva}

Saliva provides a simple, noninvasive method (compared to the traditional diagnostic fluid, blood) that can be used to detect both localized and systemic responses of inflammation [156]. Saliva does not require immediate processing or qualified personnel to collect and can be sampled multiple times in a single day without significant limitations [22]. Saliva consists of $98 \%$ water, 
but, more importantly, contains hormones, peptides, electrolytes, mucus, and various enzymes that are also found in the blood [157-158]. Numerous studies have demonstrated blood is significantly correlated to some salivary biomarkers when observing both physical and psychological stressors [22, 68-69]. While saliva provides a unique opportunity to monitor inflammatory responses, there are a number of complications that can cause an inflammatory response not necessarily related to the stressor of interest, including localized inflammation caused by gingivitis and sores/cuts. Additionally, various foods or medicines, time of day, or mouth $\mathrm{pH}$ can also affect the saliva sample [159].

\subsection{Measuring stress responses}

A vast range of experimental techniques have been established to measure biological responses, such as cellular and tissue-based biomarkers. Enzyme-linked immunosorbent assays (ELISAs) are one of the most common biomarker assays because of the ability to detect and quantify a variety of substances, including antibodies, antigens, proteins, and hormones [160].

Within this dissertation, several different multiplexed bead-based ELISAs were used to measure the biological response using a Bio-Plex 200 (flow cytometer). These assays are widely manufactured and can measure a number of different analytes including cytokines, total protein levels, and phosphorylated protein levels. Using this method is attractive due to the ability to multiplex hundreds of targets, using little sample volume, and short incubation times, while being high-throughput and maintaining high sensitivity and specificity [161].

Specifically, a bead-based ELISA manufactured by Luminex uses colored polystyrene microspheres, which can be magnetic, and are created by specific ratios of two fluorescent dyes. These beads are then conjugated to a specific capture antibody based on the particular biomarker of interest. To measure the samples, this technique uses a dual laser system which contains two 
laser beams to provide two wavelengths of excitation. In the flow cytometer, one laser (red laser $635 \mathrm{~nm}$ ) is used to identify the analyte of interest (based on the specific bead dye ratio) while the second laser (green laser $525 \mathrm{~nm}$ ) determines the concentration of the reported dye [161]. Thus, due to the unique bead regions of the microspheres and the dual laser system, one can simultaneously quantify hundreds of targets in a single well on a 96-well plate.
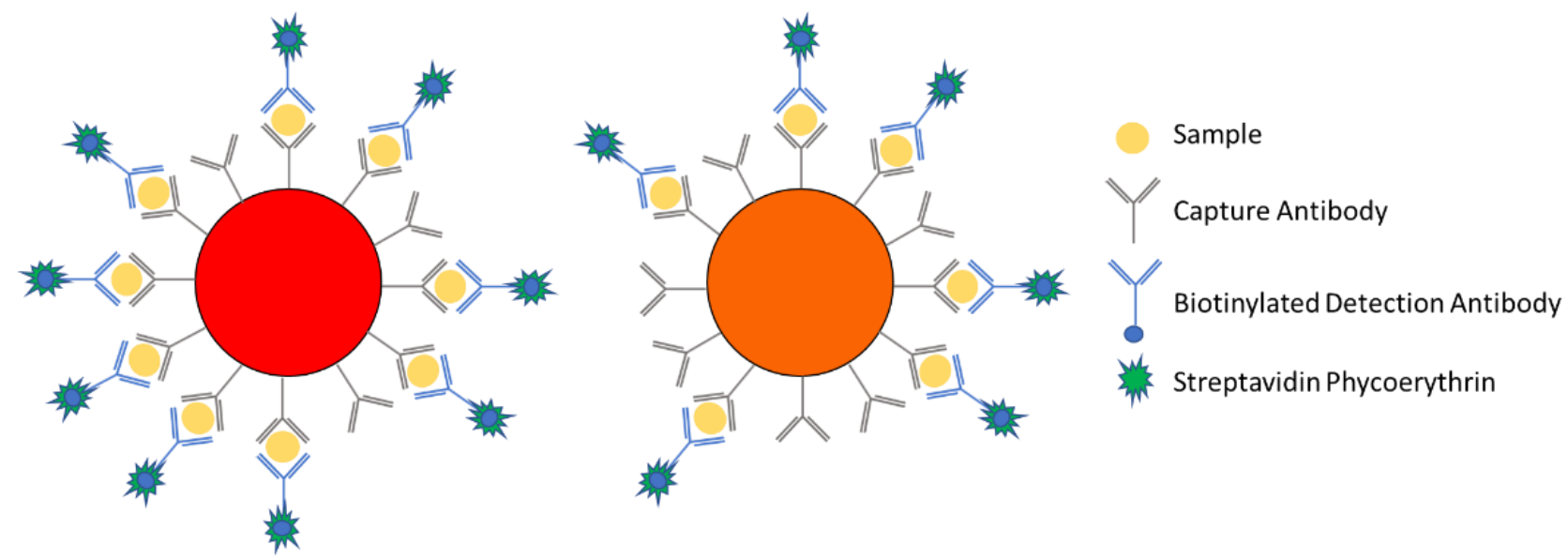

Figure 1.3. Bead-based sandwich ELISA. A polystyrene microsphere, which can magnetic, has a specific ratio of two fluorescent dyes within the bead. The assay follows a typical sandwich ELISA: the sample binds to a capture antibody that is immobilized on the bead, the biotinylated detection antibody binds to a specific epitope of the antigen, and a fluorescent reporter (streptavidin-phycoerythrin) is added. The dual laser system in the flow cytometer can then identify the bead type (for the analyte of interest) and quantify the reporter dye.

Physiological measurements (e.g., heart and respiration rates) provide real-time data that can be directly related to the biological response to stressors. As previously mentioned, the SNS is responsible for increasing both heart and breathing rates as part of the fight or flight response. Therefore, in addition to cellular biomarkers, physiological metrics can be monitored to observe a more complete response of the SNS to various stressors. Today there are numerous wearable technology sensors that can be used in human studies to constantly monitor heart rate, breathing rate, activity, etc., including smart watches and Zephyr ${ }^{\mathrm{TM}}$ sensors. The combination of data 
obtained from the physiological and biological metrics allows for a more complete understanding of the organismal response to complex stressors. 


\subsection{References}

1. Murison, R. (2016) The neurobiology of stress. In M. al'Absi \& M. A. Flaten (Eds.), The neuroscience of pain, stress, and emotion: Psychological and clinical implications (p. 29-49). Elsevier Academic Press. DOI: 10.1016/B978-0-12-800538-5.00002-9

2. McEwen, B. (2005) Stressed and stressed out: What is the difference. J. Psychiatry Neurosci., 30(5), 315-318. PMID: 16151535

3. McEwen, B. (2007) Physiology and neurobiology of stress and adaptation: Central role of the brain. Physiol. Rev., 87(3), 873-904. DOI: 10.1152/physrev.00041.2006

4. Chida, Y., Hamer, M., Wardle, J., \& Steptoe, A. (2008) Do stress-related psychosocial factors contribute to cancer incidence and survival? Nat. Clin. Pract. Oncol., 5(8), 466-475. DOI: 10.1038/ncponc 1134

5. Mariotti, A. (2015) The effects of chronic stress on health: new insights into the molecular mechanisms of brain-body communication. Future Sci. OA, 1(3), FSO23. DOI:

10.4155/fso. 15.21

6. Jackson, M. (2014) Evaluating the Role of Hans Selye in the Modern History of Stress. In D. Cantor \& E. Ramsden (Eds.), Stress, Shock, and Adaptation in the Twentieth Century. University of Rochester Press.

7. Tan, S. Y., \& Yip, A. (2018) Hans Selye (1907-1982): Founder of the stress theory. Singapore Med. J., 59(4), 170-171. DOI: 10.11622/smedj.2018043

8. Selye, H. (1956). The stress of life. New York, NY: McGraw-Hill.

9. Selye, H. (1976) Stress without Distress. In: G. Serban (Eds.), Psychopathology of Human Adaptation. Springer. DOI: 10.1007/978-1-4684-2238-2_9

10. Bienertova-Vasku, J., Lenart, P., \& Scheringer, M. (2020) Eustress and Distress: Neither Good Nor Bad, but Rather the Same? BioEssays, 42(7), 1900238. DOI: 10.1002/bies.201900238

11. Salleh, M.R. (2008) Life Event, Stress, and Illness. Malays. J. Med. Sci., 15(4), 9-18. PMID: 22589633

12. Moen, F., Myhre, K., \& Stiles, T.C. (2016) An exploration about how Passion, Perceived performance, Stress and Worries uniquely influence Athlete Burnout. J. Phys. Educ. Sports Manag., 3(1), 88-107. DOI: 10.15640/jpesm.v3n1a7

13. Salleh, M.R. (2008) Life Event, Stress and Illness. Malays. J. Med. Sci., 15(4), 9-18.

14. Chiang, J.J., Eisenberger, N.I., Seeman, T.E. \& Taylor, S.E. (2012) Negative and competitive social interactions are related to heightened proinflammatory cytokine activity. Proc. Natl. Acad. Sci. U.S.A., 109(6), 1878-1882. DOI: 10.1073/pnas.1120972109

15. Gilbert, P., McEwan, K., Bellew, R., Mills, A., \& Gale, C. (2009) The dark side of competition: How competitive behaviour and striving to avoid inferiority are linked to 
depression, anxiety, stress and self-harm. Psychol. Psychother., 82(Pt 2), 123-136. DOI: $10.1348 / 147608308 \times 379806$

16. Buser, T., Dreber, A. \& Mollerstrom, J. (2017) The impact of stress on tournament entry. Exp. Econ., 20, 506-530. DOI: 10.1007/s10683-016-9496-x

17. (2018) The cooperative human. Nat. Hum. Behav., 2, 427-428. DOI: 10.1038/s41562-0180389-1

18. Eisenberger, N., Moieni, M., Inagaki, T.K., Muscatell, K.A., \& Irwin, M.R. (2017) In Sickness and in Health: The Co-Regulation of Inflammation and Social Behavior.

Neuropsychopharmacology, 42(1), 242-253. DOI: 10.1038/npp.2016.141

19. Kirschbaum, C., Pirke, K.M., \& Hellhammer, D.H. (1993) The 'Trier Social Stress Test'--a tool for investigating psychobiological stress responses in a laboratory setting.

Neuropsychobiology, 28(1-2), 76-81. DOI: 10.1159/000119004

20. Slavich, G. M., \& Irwin, M. R. (2014) From stress to inflammation and major depressive disorder: a social signal transduction theory of depression. Psychol. Bull., 140(3), 774-815. DOI: $10.1037 / \mathrm{a} 0035302$

21. Allen, A. P., Kennedy, P. J., Dockray, S., et al. (2016) The Trier Social Stress Test: Principles and practice. Neurobiol. Stress, 6, 113-126. DOI: 10.1016/j.ynstr.2016.11.001

22. La Fratta, I., Tatangelo, R., Campagna, G., et al. (2018) The plasmatic and salivary levels of IL-1 $\beta$, IL-18 and IL-6 are associated to emotional difference during stress in young male. Sci. Rep., 8(1), 3031. DOI: 10.1038/s41598-018-21474-y

23. Dowlati, Y., Herrmann, N., Swardfager, W., et al. (2010) A meta-analysis of cytokines in major depression. Biol. Psychiatry, 67(5), 446-457. DOI: 10.1016/j.biopsych.2009.09.033

24. Denollet, J., Vrints, C.J. \& Conraads, V.M. (2008) Comparing Type D personality and older age as correlates of tumor necrosis factor-alpha dysregulation in chronic heart failure. Brain Behav. Immun., 22(5), 736-743. DOI: 10.1016/j.bbi.2007.10.015

25. Quick, J. C., \& Henderson, D. F. (2016) Occupational Stress: Preventing Suffering, Enhancing Wellbeing. Int. J. Environ. Res. Public Health, 13(5), 459. DOI: 10.3390/ijerph13050459

26. Boschetto, P., Quintavalle, S., Miotto, D., et al. (2006) Chronic obstructive pulmonary disease (COPD) and occupational exposures. J. Occup. Med. Toxicol., 1, 11. DOI: 10.1186/1745-6673-1-11

27. Chartres, N., Bero, L.A., \& Norris, S.L. (2019) A review of methods used for hazard identification and risk assessment of environmental hazards. Environ. Int., 123, 231-239. DOI: 10.1016/j.envint.2018.11.060. 
28. Judson, R., Richard, A., Dix, D. J., et al. (2009) The toxicity data landscape for environmental chemicals. Environ. Health Perspect., 117(5), 685-695. DOI:

10.1289/ehp.0800168

29. Killion, T.H., Bury, S.J., de Pontbriand, R., \& Belanich, J. (2009) United States Army Science and Technology: Sustaining Soldier Performance. Military Psychology, 21(sup1), S9S22. DOI: $10.1080 / 08995600802554540$

30. Carton, C., Friedl, K., Liljedahl, B., \& Lam, D. (2008) Chapter 10 - Health Risks During the Life Cycle of the Deployed Soldier (Publication No. RTO-TR-HFM-057). DOI: 10.14339/RTOTR-HFM-057

31. Mease, L., Sikka, R., \& Rhees, R. (2018) Pupillometer Use: Validation for Use in Military and Occupational Medical Surveillance and Response to Organophosphate and Chemical Warfare Agent Exposure. Mil. Med., 183(9-10), e291-e296. DOI: 10.1093/milmed/usy011

32. Muley, A., Shah, C., Lakhani, J., Bapna, M., \& Mehta, J. (2014) To identify morbidity and mortality predictors in acute organophosphate poisoning. Indian journal of critical care medicine : peer-reviewed, official publication of Indian J. Crit. Care Med., 18(5), 297-300. DOI: $10.4103 / 0972-5229.132488$

33. Center for Disease Control and Prevention (Obtained April 2021) Cancer Clusters. Available from: https://www.cdc.gov/nceh/clusters/fallon/organophosfaq.htm

34. Balali-Mood, M., \& Saber, H. (2012) Recent advances in the treatment of organophosphorous poisonings. Iran. J. Med. Sci., 37(2), 74-91.

35. Sugiyama, A., Matsuoka, T., Sakamune, K., et al. (2020) The Tokyo subway sarin attack has long-term effects on survivors: A 10-year study started 5 years after the terrorist incident. PloS one, 15(6), e0234967. DOI: 10.1371/journal.pone.0234967

36. Abou-Donia, M. B., Siracuse, B., Gupta, N., \& Sobel Sokol, A. (2016). Sarin (GB, Oisopropyl methylphosphonofluoridate) neurotoxicity: critical review. Crit. Rev. Toxicol., 46(10), 845-875. DOI: 10.1080/10408444.2016.1220916

37. Mawson, A. R., \& Croft, A. M. (2019) Gulf War Illness: Unifying Hypothesis for a Continuing Health Problem. Int. J. Environ. Res. Public Health, 16(1), 111. DOI: 10.3390/ijerph16010111

38. O'Callaghan, J.P., Kelly, K.A., Locker, A.R., et al. (2015) Corticosterone primes the neuroinflammatory response to DFP in mice: potential animal model of Gulf War Illness. J. Neurochem., 133(5), 708-721. DOI: 10.1111/jnc. 13088

39. Locker, A.R., Michalovicz, L.T., Kelly, K.A., et al. (2017) Corticosterone primes the neuroinflammatory response to Gulf War Illness-relevant organophosphates independently of acetylcholinesterase inhibition. J. Neurochem., 142(3), 444-455. DOI: 10.1111/jnc.14071

40. Haley, R.W., Kurt, T.L., and Hom, J. (1997) Is there a Gulf War Syndrome? Searching for syndromes by factor analysis of symptoms. JAMA, 277(3), 215-222. 
41. Dantzer, R., O’Connor, J.C., Freund, G.G., et al. (2010) From inflammation to sickness and depression: when the immune system subjugates the brain. Nat. Rev. Neurosci., 9(1), 46-56. DOI: $10.1038 / \mathrm{nrn} 2297$

42. Smith, T.C., Gray, G.C., Weir, J.C., et al. (2003) Gulf War veterans and Iraqi nerve agents at Khamisiyah: postwar hospitalization data revisited. Am. J. Epidemiol., 158(5), 457-467. DOI: 10.1093/aje/kwg178

43. J. Research Advisory Committee on Gulf War Veterans' Illnesses (2008) Gulf War Illness and the Health of Gulf War Veterans: Scientific Findings and Recommendations, Washington, D.C.

44. Brimfield, A.A. (2012) Chemicals of Military Deployments: Revisiting Gulf War Syndrome in Light of New Information. Prog. Mol. Biol. Transl., 112, 209-30. DOI: 10.1016/B978-0-12415813-9.00007-6

45. Holahan, C.J., Ragan, J.D., \& Moos, R.H. (2017) Stress. Reference Module in Neuroscience and Biobehavioral Psychology, 485-493. DOI: 10.1016/B978-0-12-809324-5.05724-2

46. Godoy, L.D., Rossignoli, M.T., Delfino-Pereira, P., Garcia-Cairasco N., de Lima Umeoka, E.H. (2018) A Comprehensive Overview on Stress Neurobiology: Basic Concepts and Clinical Implications. Front. Behav. Neurosci., 12, 127. DOI: 10.3389/fnbeh.2018.00127

47. Piazza, J. R., Almeida, D. M., Dmitrieva, N. O., \& Klein, L. C. (2010) Frontiers in the use of biomarkers of health in research on stress and aging. J. Gerontol. B. Psychol. Sci Soc. Sci., 65(5), 513-525. DOI: 10.1093/geronb/gbq049

48. Lodish, H., Berk, A., Zipursky, S.L., et al. (2000) Molecular Cell Biology. 4th edition. New York: W. H. Freeman. Section 20.3, G Protein -Coupled Receptors and Their Effectors. Available from: https://www.ncbi.nlm.nih.gov/books/NBK21718/

49. Bucsek, M. J., Giridharan, T., MacDonald, C. R., Hylander, B. L., \& Repasky, E. A. (2018) An overview of the role of sympathetic regulation of immune responses in infectious disease and autoimmunity. Int. J. Hyperthermia, 34(2), 135-143. DOI: 10.1080/02656736.2017.1411621

50. Navar, L.G. (2010) Counterpoint: Activation of the intrarenal renin-angiotensin system is the dominant contributor to systemic hypertension. J. Appl. Physiol., 109(6), 1998-2015. DOI: 10.1152/japplphysiol.00182.2010a

51. Zhang, D. Y., \& Anderson, A. S. (2014) The sympathetic nervous system and heart failure. Cardiol. Clin., 32(1), 33-vii. DOI: 10.1016/j.ccl.2013.09.010

52. Rotenberg, S., \& McGrath, J. J. (2016) Inter-relation between autonomic and HPA axis activity in children and adolescents. Biol. Psychol., 117, 16-25. DOI:

10.1016/j.biopsycho.2016.01.015

53. Stephens, M. A., \& Wand, G. (2012) Stress and the HPA axis: role of glucocorticoids in alcohol dependence. Alcohol Res., 34(4), 468-483. 
54. Thau, L., Gandhi, J., \& Sharma, S. (2021) Physiology, Cortisol. In: StatPearls [Internet]. Treasure Island (FL): StatPearls Publishing. Available from: https://www.ncbi.nlm.nih.gov/books/NBK538239/

55. Smith, S. M., \& Vale, W. W. (2006) The role of the hypothalamic-pituitary-adrenal axis in neuroendocrine responses to stress. Dialogues Clin. Neurosci., 8(4), 383-395. DOI: 10.31887/DCNS.2006.8.4/ssmith

56. Raff, H. (2016) CORT, Cort, B, Corticosterone, and now Cortistatin: Enough Already!, Endocrinology, 157(9), 3307-3308. DOI: 10.1210/en.2016-1500

57. Hannibal, K. E., \& Bishop, M. D. (2014) Chronic stress, cortisol dysfunction, and pain: a psychoneuroendocrine rationale for stress management in pain rehabilitation. Phys. Ther., 94(12), 1816-1825. DOI: $10.2522 /$ ptj.20130597

58. Uchoa, E. T., Aguilera, G., Herman, J. P., et al. (2014) Novel aspects of glucocorticoid actions. J. Neuroendocrinol., 26(9), 557-572. DOI: 10.1111/jne.12157

59. Gallo-Payet, N., \& Battista, M.C. (2014) Steroidogenesis-adrenal cell signal transduction. Compr Physiol., 4(3), 889-964. DOI: 10.1002/cphy.c130050

60. Kostadinova, F., Schwaderer, J., Sebeo, V., \& Brunner, T. (2014) Why does the gut synthesize glucocorticoids? Ann Med., 46(7), 490-497. DOI: 10.3109/07853890.2014.932920

61. Raubenheimer, P.J., Young, E.A., Andrew, R., \& Seckl, J.R. (2006) The role of corticosterone in human hypothalamic-pituitary-adrenal axis feedback. Clin. Endocrinol. (Oxf), 65(1), 22-6. DOI: $10.1111 / j .1365-2265.2006 .02540 . x$

62. Hammond, G. L. (2016) Plasma steroid-binding proteins: primary gatekeepers of steroid hormone action. J. Endocrinol., 230(1), R13-R25. DOI: 10.1530/JOE-16-0070

63. Herold, M. J., McPherson, K. G., \& Reichardt, H. M. (2006) Glucocorticoids in T cell apoptosis and function. Cell. Mol. Life Sci., 63(1), 60-72. DOI: 10.1007/s00018-005-5390-y

64. Levine, A., Zagoory-Sharon, O., Feldman, R., Lewis, J.G., \& Weller, A. (2007) Measuring cortisol in human psychobiological studies. Physiol. Behav., 90(1), 43-53. DOI:

10.1016/j.physbeh.2006.08.025

65. Lee, D. Y., Kim, E., \& Choi, M. H. (2015) Technical and clinical aspects of cortisol as a biochemical marker of chronic stress. BMB Rep., 48(4), 209-216. DOI:

10.5483/bmbrep.2015.48.4.275

66. Rotenberg, S., \& McGrath, J. J. (2016). Inter-relation between autonomic and HPA axis activity in children and adolescents. Biol. Psychol., 117, 16-25. DOI:

10.1016/j.biopsycho.2016.01.015

67. Segerstrom, S. C., \& Miller, G. E. (2004) Psychological stress and the human immune system: a meta-analytic study of 30 years of inquiry. Psychol. Bull., 130(4), 601-630. DOI: 10.1037/0033-2909.130.4.601 
68. Nater, U.M. \& Rohleder, N. (2009) Salivary alpha-amylase as a non-invasive biomarker for the sympathetic nervous system: current state of research. Psychoneuroendocrinology, 34(4), 486-496. DOI: 10.1016/j.psyneuen.2009.01.014

69. Nunes, L.A., Brenzikofer, R. \& Macedo, D.V. (2011) Reference intervals for saliva analytes collected by a standardized method in a physically active population. Clin. Biochem., 44(17-18), 1440-1444. DOI: 10.1016/j.clinbiochem.2011.09.012

70. Chen, L., Deng, H., Cui, H., et al. (2017) Inflammatory responses and inflammationassociated diseases in organs. Oncotarget, 9(6), 7204-7218. DOI: 10.18632/oncotarget.23208

71. Morey, J. N., Boggero, I. A., Scott, A. B., \& Segerstrom, S. C. (2015) Current Directions in Stress and Human Immune Function. Curr. Opin. Psychol., 5, 13-17. DOI:

10.1016/j.copsyc.2015.03.007

72. Gouin, J.P., Glaser, R., Malarkey, W.B., Beversdorf, D., \& Kiecolt-Glaser, J. (2012) Chronic stress, daily stressors, and circulating inflammatory markers. Health Psychol., 31(2), 264-268. DOI: $10.1037 / \mathrm{a} 0025536$

73. Ershler, W.B. (1993) Interleukin-6: a cytokine for gerontologists. J. Am. Geriatr. Soc., 41(2), 176-181. DOI: 10.1111/j.1532-5415.1993.tb02054.x

74. Alberts, B., Johnson, A., Lewis, J., et al. (2002) Molecular Biology of the Cell. 4th edition. New York: Garland Science. Chapter 24, The Adaptive Immune System. Available from: https://www.ncbi.nlm.nih.gov/books/NBK21070/

75. Relja, B., \& Land, W.G. (2020) Damage-associated molecular patterns in trauma. Eur. J. Trauma Emerg. Surg., 46, 751-775. DOI: 10.1007/s00068-019-01235-w

76. Zhang, J. M., \& An, J. (2007) Cytokines, inflammation, and pain. Int. Anesthesiol. Clin., 45(2), 27-37. DOI: 10.1097/AIA.0b013e318034194e

77. Dinarello, C. A. (2007) Historical insights into cytokines. Eur. J. Immunol., 37 Suppl 1(Suppl 1), S34-S45. DOI: 10.1002/eji.200737772

78. Kishimoto, T. (2006) Interleukin-6: discovery of a pleiotropic cytokine. Arthritis Res. Ther., 8, S2. DOI: $10.1186 / \operatorname{ar} 1916$

79. Leonard. W.J. Type I Cytokines and Interferons and Their Receptors, In W.E. Paul (Eds.), Fundamental Immunology, Fifth Edition. Lippincott Williams \& Wilkins.

80. Cameron, M.J., \& Kelvin, D.J. (2000) Cytokines, Chemokines and Their Receptors. In:

Madame Curie Bioscience Database [Internet]. Austin (TX): Landes Bioscience. Available from: https://www.ncbi.nlm.nih.gov/books/NBK6294/

81. Bao, K., \& Reinhardt, R. L. (2015) The differential expression of IL-4 and IL-13 and its impact on type-2 immunity. Cytokine, 75(1), 25-37. DOI: 10.1016/j.cyto.2015.05.008 
82. Cicchese, J. M., Evans, S., Hult, C., et al. (2018) Dynamic balance of pro- and antiinflammatory signals controls disease and limits pathology. Immunol. Rev., 285(1), 147-167. DOI: $10.1111 /$ imr.12671

83. Scheller, J., Chalaris, A., Schmidt-Arras, D., \& Rose-John, S. (2011) The pro- and antiinflammatory properties of the cytokine interleukin-6. Biochim. Biophys. Acta., 1813(5), 87888. DOI: 10.1016/j.bbamcr.2011.01.034

84. Justiz Vaillant, A.A., \& Qurie, A. (2021) Interleukin. [Updated 2020 Aug 30]. In: StatPearls [Internet]. Treasure Island (FL): StatPearls Publishing. Available from:

https://www.ncbi.nlm.nih.gov/books/NBK499840/

85. Ryff, J.C., \& Pestka, S. (2013) Interferons and Interleukins. In: Crommelin, D., Sindelar, R., Meibohm, B. (eds) Pharmaceutical Biotechnology. Springer, New York, NY. DOI: 10.1007/9781-4614-6486-0_21

86. Ivashkiv, L. B., \& Donlin, L. T. (2014) Regulation of type I interferon responses. Nat. Rev. Immunol., 14(1), 36-49. DOI: 10.1038/nri3581

87. McNab, F., Mayer-Barber, K., Sher, A., Wack, A., \& O'Garra, A. (2015) Type I interferons in infectious disease. Nat. Rev. Immunol., 15(2), 87-103. DOI: 10.1038/nri3787

88. Nan, Y., Wu, C., \& Zhang, Y.J. (2017) Interplay between Janus Kinase/Signal Transducer and Activator of Transcription Signaling Activated by Type I Interferons and Viral Antagonism. Front. Immunol., 8, 1758. DOI: 10.3389/fimmu.2017.01758

89. Horvath, C.M. (2004) The Jak-STAT pathway stimulated by interferon gamma. Sci. STKE, 2004(260), tr8. DOI: 10.1126/stke.2602004tr8

90. Byng-Maddick, R., Turner, C.T., Pollara, G., et al. (2017) Tumor Necrosis Factor (TNF) Bioactivity at the Site of an Acute Cell-Mediated Immune Response Is Preserved in Rheumatoid Arthritis Patients Responding to Anti-TNF Therapy. Front. Immunol., 8, 932. DOI:

10.3389/fimmu.2017.00932

91. Wang, X., \& Lin, Y. (2008) Tumor necrosis factor and cancer, buddies or foes?. Acta Pharmacol. Sin., 29(11), 1275-1288. DOI: 10.1111/j.1745-7254.2008.00889.x

92. Aggarwal, B. (2003) Signalling pathways of the TNF superfamily: a double-edged sword. Nat. Rev. Immunol., 3, 745-756. DOI: 10.1038/nri1184

93. Hedayat, M., Mahmoudi, M.J., Rose, N.R. et al. (2010) Proinflammatory cytokines in heart failure: double-edged swords. Heart Fail. Rev., 15, 543-562. DOI: 10.1007/s10741-010-9168-4

94. Metcalf, D. (2013) The colony-stimulating factors and cancer. Cancer Immunol. Res., 1(6), 351-356. DOI: 10.1158/2326-6066.CIR-13-0151

95. Hamilton, J. (2008) Colony-stimulating factors in inflammation and autoimmunity. Nat. Rev. Immunol., 8, 533-544. DOI: 10.1038/nri2356 
96. Trapnell, B.C., \& Abe, S. (2006) Colony Stimulating Factors. In Laurent, G.J., Shapiro, S.D. (eds.) Encyclopedia of Respiratory Medicine, 540-546. Academic Press. DOI: 10.1016/B0-12370879-6/00093-4

97. Andersen, M.E. (2010) Calling on science: making "alternatives" the new gold standard. ALTEX, 27(2), 135-143. DOI: 10.14573/altex.2010.2.135

98. Rosenbaum, D. M., Rasmussen, S. G., \& Kobilka, B. K. (2009) The structure and function of G-protein-coupled receptors. Nature, 459(7245), 356-363. DOI: 10.1038/nature08144

99. Zhao, J., Deng, Y., Jiang, Z., \& Qing, H. G. (2016) Protein-Coupled Receptors (GPCRs) in Alzheimer's Disease: A Focus on BACE1 Related GPCRs. Front. Aging Neurosci., 8, 58. DOI: 10.3389/fnagi.2016.00058.

100. Manglik, A., \& Kobilka, B. (2014) The role of protein dynamics in GPCR function: insights from the $\beta 2 A R$ and rhodopsin. Curr. Opin. Cell Biol., 27, 136-143. DOI:

10.1016/j.ceb.2014.01.008

101. Boyd, J.W. \& Neubig, R. (2019) Cellular Signal Transduction in Toxicology and Pharmacology: Data Collection, Analysis, and Interpretation, John Wiley \& Sons, Ltd.

102. Serezani, C. H., Ballinger, M. N., Aronoff, D. M., \& Peters-Golden, M. (2008) Cyclic AMP: master regulator of innate immune cell function. Am. J. Respir. Cell Mol. Biol., 39(2), 127-132. DOI: 10.1165/rcmb.2008-0091TR

103. Taskén, K., \& Aandahl, E.M. (2004) Localized effects of cAMP mediated by distinct routes of protein kinase A. Physiol Rev., 84(1), 137-167. DOI: 10.1152/physrev.00021.2003

104. Shaywitz, A.J., \& Greenberg, M.E. (1999) CREB: a stimulus-induced transcription factor activated by a diverse array of extracellular signals. Annu. Rev. Biochem., 68, 821-861. DOI: 10.1146/annurev.biochem.68.1.821

105. Wen, A. Y., Sakamoto, K. M., \& Miller, L. S. (2010) The role of the transcription factor CREB in immune function. J. Immunol., 185(11), 6413-6419. DOI: 10.4049/jimmunol.1001829

106. Lonze, B.E., \& Ginty, D.D. (2002) Function and regulation of CREB family transcription factors in the nervous system. Neuron., 35(4), 605-623. DOI: 10.1016/s0896-6273(02)00828-0

107. Smart, R.C. \& Hodgson, E. (2007) Molecular and Biochemical Toxicology, Fourth Edition, John Wiley \& Sons, Inc. DOI: 10.1002/9780470285251

108. Ardito, F., Giuliani, M., Perrone, D., Troiano, G., \& Lo Muzio, L. (2017) The crucial role of protein phosphorylation in cell signaling and its use as targeted therapy (Review). Int. J. Mol. Med., 40(2), 271-280. DOI: 10.3892/ijmm.2017.3036

109. Huse, M., \& Kuriyan, J. (2002) The conformational plasticity of protein kinases. Cell., 109(3), 275-282. DOI: 10.1016/s0092-8674(02)00741-9

110. Deribe, Y.L., Pawson, T., Dikic, I. (2010) Post-translational modifications in signal integration. Nat. Struct. Mol. Biol., 17(6), 666-672. DOI: 10.1038/nsmb.1842 
111. Nurse, P. (2000) A long twentieth century of the cell cycle and beyond. Cell., 100(1), 71-78. DOI: $10.1016 / \mathrm{s} 0092-8674(00) 81684-0$.

112. Ullrich, A., \& Schlessinger, J. (1990) Signal transduction by receptors with tyrosine kinase activity. Cell., 61(2), 203-212. DOI: 10.1016/0092-8674(90)90801-k

113. Morrison, D. K. (2012) MAP kinase pathways. Cold Spring Harb. Perspect. Biol., 4(11), a011254. DOI: $10.1101 /$ cshperspect.a011254

114. Chang, F., Steelman, L.S., Lee, J.T., et al. (2003) Signal transduction mediated by the Ras/Raf/MEK/ERK pathway from cytokine receptors to transcription factors: potential targeting for therapeutic intervention. Leukemia, 17(7), 1263-1293. DOI: 10.1038/sj.leu.2402945

115. Geest, C.R., Buitenhuis, M., Groot Koerkamp, M.J., et al. (2009) Tight control of MEKERK activation is essential in regulating proliferation, survival, and cytokine production of CD34+-derived neutrophil progenitors. Blood, 114(16), 3402-3412. DOI: 10.1182/blood-200808-175141

116. Zou, J., Lei, T., Guo, P., et al. (2019) Mechanisms shaping the role of ERK1/2 in cellular senescence (Review). Mol. Med. Rep., 19(2), 759-770. DOI: 10.3892/mmr.2018.9712

117. Meloche, S., \& Pouysségur, J. (2007) The ERK1/2 mitogen-activated protein kinase pathway as a master regulator of the G1- to S-phase transition. Oncogene 26, 3227-3239. DOI: 10.1038/sj.onc. 1210414

118. Li, L., Zhao, G. D., Shi, Z., et al. (2016) The Ras/Raf/MEK/ERK signaling pathway and its role in the occurrence and development of HCC. Oncol. Lett., 12(5), 3045-3050. DOI: 10.3892/ol.2016.5110

119. Wagner, E., \& Nebreda, Á. (2009) Signal integration by JNK and p38 MAPK pathways in cancer development. Nat. Rev. Cancer, 9, 537-549. DOI: 10.1038/nrc2694

120. Zhang, W., \& Liu, H. (2002) MAPK signal pathways in the regulation of cell proliferation in mammalian cells. Cell. Res., 12, 9-18. DOI: 10.1038/sj.cr.7290105

121. Tournier, C., Whitmarsh, A. J., Cavanagh, J., Barrett, T., \& Davis, R. J. (1997) Mitogenactivated protein kinase kinase 7 is an activator of the c-Jun NH2-terminal kinase. Proc. Natl. Acad. Sci. U.S.A., 94(14), 7337-7342. DOI: 10.1073/pnas.94.14.7337

122. Guma, M., \& Firestein, G. S. (2012) c-Jun N-Terminal Kinase in Inflammation and Rheumatic Diseases. Open Rheumatol. J., 6, 220-231. DOI: 10.2174/1874312901206010220

123. Zarubin, T., \& Han, J. (2005) Activation and signaling of the p38 MAP kinase pathway. Cell Res., 15, 11-18. DOI: 10.1038/sj.cr.7290257

124. Xu, F., Na, L., Li, Y. et al. (2020) Roles of the PI3K/AKT/mTOR signalling pathways in neurodegenerative diseases and tumours. Cell Biosci., 10, 54. DOI: 10.1186/s13578-020-004160 
125. Fruman, D. A., Chiu, H., Hopkins, B. D., et al. (2017) The PI3K Pathway in Human Disease. Cell, 170(4), 605-635. DOI: 10.1016/j.cell.2017.07.029

126. Yang, W. L., Wu, C. Y., Wu, J., \& Lin, H. K. (2010) Regulation of Akt signaling activation by ubiquitination. Cell Cycle, 9(3), 487-497. DOI: 10.4161/cc.9.3.10508

127. Manning, B. D., \& Toker, A. (2017) AKT/PKB Signaling: Navigating the Network. Cell, 169(3), 381-405. DOI: 10.1016/j.cell.2017.04.001

128. Hoxhaj, G., \& Manning, B.D. (2020) The PI3K-AKT network at the interface of oncogenic signalling and cancer metabolism. Nat. Rev. Cancer, 20, 74-88. DOI: 10.1038/s41568-0190216-7

129. Hemmings, B. A., \& Restuccia, D. F. (2012). PI3K-PKB/Akt pathway. Cold Spring Harb. Perspect. Biol., 4(9), a011189. DOI: 10.1101/cshperspect.a011189

130. Vert, G., \& Chory, J. (2011) Crosstalk in cellular signaling: background noise or the real thing?. Dev. Cell, 21(6), 985-991. DOI: 10.1016/j.devcel.2011.11.006

131. Rowland, M., Greenbaum, J. \& Deeds, E. (2017) Crosstalk and the evolvability of intracellular communication. Nat. Commun., 8, 16009. DOI: 10.1038/ncomms16009

132. Mendoza, M.C., Er, E.E., \& Blenis, J. (2011) The Ras-ERK and PI3K-mTOR pathways: cross-talk and compensation. Trends Biochem. Sci., 36(6), 320-328. DOI:

10.1016/j.tibs.2011.03.006

133. Loo, L.H., Bougen-Zhukov, N.M., \& Tan, W.L.C. (2017) Early spatiotemporal-specific changes in intermediate signals are predictive of cytotoxic sensitivity to TNF $\alpha$ and co-treatments. Sci. Rep., 7, 43541. DOI: 10.1038/srep43541

134. Berridge, M.J., Cobbold, P.H., \& Cuthbertson, K.S. (1998) Spatial and temporal aspects of cell signalling. Philos. Trans. R. Soc. Lond. B. Biol. Sci., 320(1199), 325-43. DOI:

10.1098/rstb.1988.0080

135. Vrana, J.A., Currie, H.N., Han, A.A., \& Boyd, J. (2014) Forecasting Cell Death DoseResponse from Early Signal Transduction Responses In Vitro. Toxicol. Sci., 140(2), 338-351. DOI: $10.1093 /$ toxsci/kfu089

136. Rehm, M., Huber, H.J., Hellwig, C.T., et al. (2009) Dynamics of outer mitochondrial membrane permeabilization during apoptosis. Cell Death Differ., 16(4), 613-623. DOI: 10.1038/cdd.2008.187

137. Lemasters, J.J., Nieminen, A.L., Qian, T., et al. (1998) The mitochondrial permeability transition in cell death: a common mechanism in necrosis, apoptosis and autophagy. Biochim. Biophys. Acta., 1366(1-2), 177-196. DOI: 10.1016/s0005-2728(98)00112-1

138. Messam, C.A., \& Pittman, R.N. (1998) Asynchrony and commitment to die during apoptosis. Exp. Cell. Res., 238(2), 389-398. DOI: 10.1006/excr.1997.3845 
139. Gupta, R. C. (2004). Brain regional heterogeneity and toxicological mechanisms of organophosphates and carbamates. Toxicol. Mech. Methods, 14(3), 103-1080. DOI:

$10.1080 / 15376520490429175$

140. Prince, N., Penatzer, J.A., Dietz, M.J., Boyd, J.W. (2020) Impact of cytokines and phosphoproteins in response to chronic joint infection. Biology, 9(7), 167. DOI:

10.3390/biology9070167

141. Prince, N., Penatzer, J.A., Dietz, M.J., Boyd, J.W. (2020) Localized cytokine responses to total knee arthroplasty and total knee revision complications. J. Transl. Med., 18(1), 330. DOI: 10.1186/s12967-020-02510-w

142. Han, A.A., Currie, H.N., Loos, M.S., et al. (2018) The impact of cytokine responses in the intra- and extracellular signaling network of a traumatic injury. Cytokine, 106, 136-147. DOI: 10.1016/j.cyto.2017.10.027

143. National Research Council (US) Committee on Environmental Epidemiology; National Research Council (US) Commission on Life Sciences. (1997) Environmental Epidemiology: Volume 2: Use of the Gray Literature and Other Data in Environmental Epidemiology. Washington (DC): National Academies Press (US). Exposure Assessment in Environmental Epidemiology. Available from: https://www.ncbi.nlm.nih.gov/books/NBK233635/

144. Pedrete, T.A., Mota, C.L., Gonçalves, E.S., \& Moreira, J.C. (2016) Towards a personalized risk assessment for exposure of humans to toxic substances. Cadernos Saúde Coletiva, 24(2), 262-273. DOI: 10.1590/1414-462X201600020135

145. Cohen Hubal, E.A., Wetmore, B.A., Wambaugh, J.F. et al. (2019) Advancing internal exposure and physiologically-based toxicokinetic modeling for 21 st-century risk assessments. J. Expo. Sci. Environ. Epidemiol., 29, 11-20. DOI: 10.1038/s41370-018-0046-9

146. Murthy, V. (2017) A Nation Under Pressure: The Public Health Consequences of Stress in America. National Center for Complementary and Integrative Health. Available from:

https://www.nccih.nih.gov/news/events/a-nation-under-pressure-the-public-health-consequencesof-stress-in-america

147. American Psychological Association. (2020) Stress in America 2020: A National Mental Health Crisis. Available from: https://www.apa.org/news/press/releases/stress/2020/reportoctober

148. Turner, P. V., Brabb, T., Pekow, C., \& Vasbinder, M. A. (2011) Administration of substances to laboratory animals: routes of administration and factors to consider. J. Am. Assoc. Lab. Anim. Sci., 50(5), 600-613.

149. Pond, S.M., \& Tozer, T.N. (1984) First-Pass Elimination Basic Concepts and Clinical Consequences. Clin. Pharmacokinet., 9, 1-25. DOI: 10.2165/00003088-198409010-00001 
150. Herman, T.F., \& Santos, C. (2020) First Pass Effect. In: StatPearls [Internet]. Treasure Island (FL): StatPearls Publishing. Available from:

https://www.ncbi.nlm.nih.gov/books/NBK551679/

152. Vaught, J.B., \& Henderson, M.K. (2011) Biological sample collection, processing, storage and information management. IARC Sci. Publ., 163, 23-42. PMID: 22997855.

153. Shomer, N. H., Allen-Worthington, K. H., Hickman, D. L., et al. (2020) Review of Rodent Euthanasia Methods. J. Am. Assoc. Lab. Anim. Sci., 59(3), 242-253. DOI: 10.30802/AALASJAALAS-19-000084

154. O'Callaghan, J.P., \& Sriram, K. (2004) Focused microwave irradiation of the brain preserves in vivo protein phosphorylation: Comparison with other methods of sacrifice and analysis of multiple phosphoproteins. J. Neurosci. Methods, 135(1-2), 159-168. DOI: 10.1016/j.jneumeth.2003.12.006

155. Shabihkhani, M., Lucey, G. M., Wei, B., et al. (2014) The procurement, storage, and quality assurance of frozen blood and tissue biospecimens in pathology, biorepository, and biobank settings. Clin. Biochem., 47(4-5), 258-266. DOI: 10.1016/j.clinbiochem.2014.01.002

156. Rathnayake, N., Åkerman, S., Klinge, B., et al. (2013) Salivary Biomarkers for Detection of Systemic Diseases. PLoS ONE, 8(4), e61356. DOI: 10.1371/journal.pone.0061356

157. Nunes, L. A., Mussavira, S., \& Bindhu, O. S. (2015) Clinical and diagnostic utility of saliva

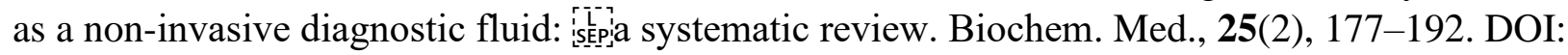
10.11613/BM.2015.018

158. Papacoasta, E. \& Nassis, G.P. (2011) Saliva as a tool for monitoring steroid, peptide and immune markers in sport and exercise science. J. Sci. Med. Sport, 14(5), 424-434. DOI: 10.1016/j.jsams.2011.03.004

159. Hofman, L.F. (2001) Human saliva as a diagnostic specimen. J Nutr., 131(5), 1621S-1625S. DOI: $10.1093 / \mathrm{jn} / 131.5 .1621 \mathrm{~S}$

160. Alhajj, M., \& Farhana, A. (2021) Enzyme Linked Immunosorbent Assay. In: StatPearls [Internet]. Treasure Island (FL): StatPearls Publishing. Available from:

https://www.ncbi.nlm.nih.gov/books/NBK555922/

161. Houser, B. (2012) Bio-Rad's Bio-Plex® suspension array system, xMAP technology overview. Arch. Physiol. Biochem., 118(4), 192-196. DOI: 10.3109/13813455.2012.705301 


\section{Chapter 2: Salivary cytokines as a biomarker of social stress in a mock rescue mission}

Parts of this chapter have been published previously: Julia A. Penatzer, Julie V. Miller, Alice A. Han, Nicole Prince, Jonathan W. Boyd (2020) Salivary cytokines as a biomarker of social stress in a mock rescue mission. Brain, Behavior and Immunity-Health, 4, 100068. https://doi.org/10.1016/j.bbih.2020.100068

Using salivary inflammatory markers as a noninvasive biomonitoring technique within natural social contexts has become increasingly important to link social and biological responses. Many studies have associated circulating cytokines to distinct aspects of physical activity and social/emotional behavior; however, they have not been linked to success and failure in a naturalistic setting for military personnel performing tasks. In this study, salivary cytokines were studied in a group of fifteen Air Force Reserve Officers' Training Corps (ROTC; 14 males, 1 female) subjects performing three mock hostage rescue missions, designed to prompt responses associated with baseline, success, and failure. Each subject completed the tasks of the mission individually and again in randomly assigned teams. Participants were outfitted via direct skin

contact with comfortable external Zephyr ${ }^{\mathrm{TM}}$ sensors to monitor heart rate, breathing rate, and activity while completing each task. Saliva samples were collected before and after the completion of each mission, and cytokine levels were quantified using enzyme-linked immunosorbent assay (ELISA) beads. These biomarkers were used to describe the body's immune response to success and failure when performing a mock rescue mission individually and in a team. All measured cytokine levels increased following failed missions performed individually, compared to cytokine levels associated with successful missions. When completing the tasks as a team, there were no significant differences in cytokine response between success and failure; however, being in a team stimulated an increased pre-mission cytokine response, suggesting the concept of teamwork and 
performing with peers for the first time had a more significant impact than the notion of failing. Additionally, none of the cytokines tested for individual missions correlated to physical activity markers (heart rate, breathing rate, activity) measured during performance. These results indicate a potentially new noninvasive method of determining social stress levels under taxing conditions.

\subsection{Introduction}

Social settings are known to stimulate immune responses related to stress and inflammation [1-3]. In the presence of stress-induced stimuli, the communication between the brain and the body occurs through the autonomic nervous system, as well as the endocrine and immune systems [4-5]. This communication relies on the release of dynamic biochemical messengers to promote adaptation in response to social, mental, and physical stressors [6]. When these messengers are triggered, they can be measured as early as minutes after an individual perceives the stimuli [7].

While measuring these biochemical messengers is critical to understanding the dynamic biological cascades associated with social, mental, and physical stressors, research has been limited because temporal data can be restricted if solely relying on blood as a sample. Sampling with saliva provides a simple, noninvasive method, as an alternative to serum samples, that does not require immediate processing or qualified personnel to collect [1]. Moreover, multiple saliva collections can be obtained on the same day and repeated over time without significant limitations. Saliva consists of $98 \%$ water that contains hormones, peptides, electrolytes, mucus, and various enzymes that are also found in the blood [8-9]. Recent studies have demonstrated blood, the standard diagnostic fluid, is significantly correlated to some salivary biomarkers while monitoring physical activity as well as psychological stress [1, 10-11]. 
Cytokines are soluble glycoproteins secreted by immune cells and found in both blood and saliva samples $[1,12-13]$. They have been extensively studied in relation to physical activity and short-term exercise, which affects both local and systemic production in response to muscle fatigue [14-15]. More importantly, several studies have examined the effects of social stressors on cytokines [1, 16]. Dickerson et al. (2004a), demonstrated that individuals who wrote about an experience that induced feelings of shame displayed an increased inflammatory cytokine response versus those who wrote about a control topic [17-18]. Emotional states created by social ties, such as anxiety, anger, and depression, also have been shown to increase proinflammatory cytokines [1, 19-20]. Chiang et al. (2012) demonstrated that individuals who experienced both negative and competitive social interactions displayed heightened proinflammatory cytokines [16]. Additionally, studies have shown social defeat promotes an increase in cytokine response [21-23]. Thus, cytokines provide a new objective and quantifiable biological target that may be explored in relation to success and failure as a response to either physical or social stress.

To explore this concept, Air Force Reserve Officers' Training Corps (ROTC) cadets were enrolled to perform a mock hostage rescue mission individually, and later in teams. Subjects wore an external Zephyr ${ }^{\mathrm{TM}}$ (Zephyr Performance System, Boulder, CO) monitor that collected physical activity data during all of the missions. A saliva sample was collected from each subject before the first mission and after the completion of each mission. Cytokine levels were measured using a standard enzyme-linked immunosorbent assay (ELISA) kit. In contrast to current studies, the present research combines physical activity with salivary cytokine concentrations for individuals in response to both successful and failed missions, while performing as individuals and in teams. 


\subsection{Materials and methods}

\subsubsection{Participants}

This study received approval from the Institutional Review Board (IRB) committee at West Virginia University (IRB \#1511920378) and the United States Army Medical Research and Materiel Command (USAMRMC; IRB \#H-24174). Air Force Reserve Officers' Training Corps (ROTC) cadets were enrolled to perform a mock hostage rescue mission. The subjects were introduced to the study during a university ROTC course. A total of 16 subjects volunteered and provided consent for the study; however, one subject failed to provide enough sample to test and was removed from the analysis. Therefore, a homogenous cohort of 15 ROTC cadets (14 males, 1 female) were considered for statistical analyses (Table 2.1).

Table 2.1. Summary of subjects from mock crime scene house. Displayed are the means and standard deviation (SD) from the subjects.

\begin{tabular}{|l|l|l|l|}
\hline & Age (years) & Body Mass Index $\left(\mathrm{kg} \bullet \mathrm{m}^{-2}\right)$ & Body Fat \% \\
\hline Mean \pm SD & $19.53 \pm 1.1$ & $23.95 \pm 2.8$ & $15.52 \pm 6.0$ \\
\hline
\end{tabular}

\subsubsection{Procedure}

Three missions were completed by each individual and, on a separate day, by each team. The subject was given the following mission to read:

"One of the houses outside is a known terrorist stronghold and possible bombmaking facility. After monitoring the facility for some time, we have determined their occupancy habits. At this time, we know the structure to be vacant. It is, however, believed to have a hostage inside; this hostage is believed to know valuable information and to be sympathetic to our cause. The objective of this mission is to carefully canvass the building, which means leaving no evidence of your presence behind (leave all belongings inside the way you found it, lights off, doors closed), and search each room to find and rescue the 
hostage (punching bag). It is believed that the hostage has been injured and is possibly unconscious. Our preliminary surveillance has shown that there is a backboard, hand cart, and four bungee cords spread out somewhere in the building. You must find all the items, figure out how to assemble all the items together, and carefully transport the hostage back to the rendezvous point at the garage. I must stress the fact that the building must be left in the state you found it, therefore, move quickly and quietly in the building and use caution when entering and searching each room."

During the first mission, the subjects/teams were instructed to complete the mission while being timed to familiarize the participant with the crime scene house and the task at hand (baseline mission). For the second rescue mission, the subjects/teams were instructed to go as fast as they could, but still abide by the mission objectives and rules (considered a successful mission). During the third hostage rescue mission, the subjects/teams were informed that they would be timed again; however, this time, one minute into the mission, an air horn was blown and the mission director stated, "Insurgents are returning: You have one minute left or you will be captured!" The second horn blew one minute after the first horn and the mission director yelled "FAIL! Assemble the hostage and come back to the rendezvous point" (considered a failed mission). The timer was started for each mission as soon as the subject/team entered the house and ended as soon as they came back to the rendezvous spot and was recorded in seconds. For each mission, all four items (hostage, backboard, hand cart, bungee cords) were randomly assigned to different locations in the house.

\subsubsection{Physical activity markers}

Participants were outfitted via direct skin contact with comfortable external Zephyr ${ }^{\mathrm{TM}}$ sensors (Zephyr Performance Systems) to continuously monitor heart rate, breathing rate, and 
activity levels while completing the mission. Heart rate was measured by a sensor in the strap which detects heart electrocardiogram signals, while breathing rate was measured via a pressure pad to detect the expansion of the rib cage, and an internal accelerometer measured the subject activity [24]. Physical activity markers were calculated using the summed physical activity level (i.e., heart rate) while completing the mission normalized to the time to complete the mission (i.e., beats/second). As previously mentioned, the timer was started as soon as the subject/team entered the house and ended as soon as the subject(s) came back to the rendezvous spot.

\subsubsection{Saliva samples}

Saliva samples were collected before the first mission (baseline; approximately 19:00) and after the completion of each mission (baseline, success, and failure). Prior to saliva collection, subjects were given an $8 \mathrm{oz}$ bottle of water and instructed to immediately drink it. Ten minutes after each subject finished their water, a 1-mL sample was taken with the help of a saliva collection aid (Salimetrics, Carlsbad, CA). Immediately after collection, the saliva samples were stored on ice and later transferred to a freezer $\left(-80^{\circ} \mathrm{C}\right)$ until analysis. On the day of analysis, samples were centrifuged at $3000 \mathrm{rpm}$ for 15 minutes at $4^{\circ} \mathrm{C}$.

\subsubsection{Cytokine 10-plex assay}

The saliva supernatant was assayed for the following cytokines via an ultrasensitive human

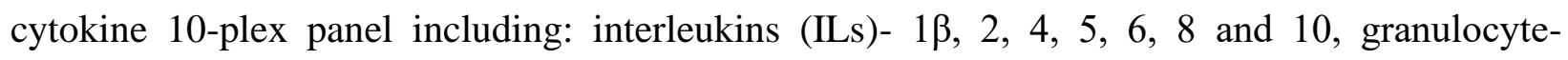
macrophage colony-stimulating factor (GM-CSF), tumor necrosis factor alpha (TNF- $\alpha$ ), and interferon gamma (IFN- $\gamma$ ) (Fisher Scientific, Pittsburgh, PA). The samples were analyzed with the Bio-Plex 200 suspension array system and Pro II Wash Station (Bio-Rad, Hercules, CA), according to the manufacturer's instructions and performed in triplicate (inter assay variation $<10 \%)$. 


\subsubsection{Statistical analysis}

All data analyses were performed using GraphPad Prism V5 (San Diego, CA) and SAS JMP Pro V14 (Cary, NC). The samples were quantified using the provided standards from the kit. Two cytokine targets, IL-1 $\beta$ and IL-8, were consistently above the highest standard for all participants and were removed from the dataset. Responses from the female subject did not significantly affect the analyses and therefore was included in all of the datasets. Data are presented as means \pm standard error of the mean (SEM), and significance tests were performed using the Friedman test or the Wilcoxon matched-pairs signed rank test, where appropriate. Dunn's posttest was also performed to determine significant changes in cytokine levels across missions. Values were considered statistically significant at a $5 \%$ level of significance $(\mathrm{p}<0.05)$. Pearson correlation analyses were also conducted for cytokine and physical activity marker data; however, none of the correlations were considered statistically significant at a $5 \%$ level of significance $(p>0.05)$ (data not shown). Additionally, Pearson correlations were run between cytokine concentrations and age, body fat percentage, and body mass index (BMI) for each subject to determine the possible contribution of these variables. None of the correlations between cytokine levels and age, body fat percentage, or BMI were significant ( $\mathrm{p}>0.05)$ (data not shown).

\subsection{Results}

\subsubsection{Physical activity markers of stress}

Using external Zephyr ${ }^{\mathrm{TM}}$ sensors, the physical activity markers (heart rate, breathing rate, and activity) were calculated using the summed marker normalized by the time (seconds) it took to complete the mission. There was a significant increase in activity for individuals compared to baseline for success and failure $(\mathrm{p}<0.05)$. Conversely, there was no significant difference for success and failure for individuals or for teams for heart rate, breathing rate, and activity ( $\mathrm{p}>0.05)$ 
(Figure 2.1). However, heart rate and activity were significantly decreased for individuals in a team compared to individual tasks for failed missions. Heart rate was also significantly decreased for individuals in a team for successful missions.
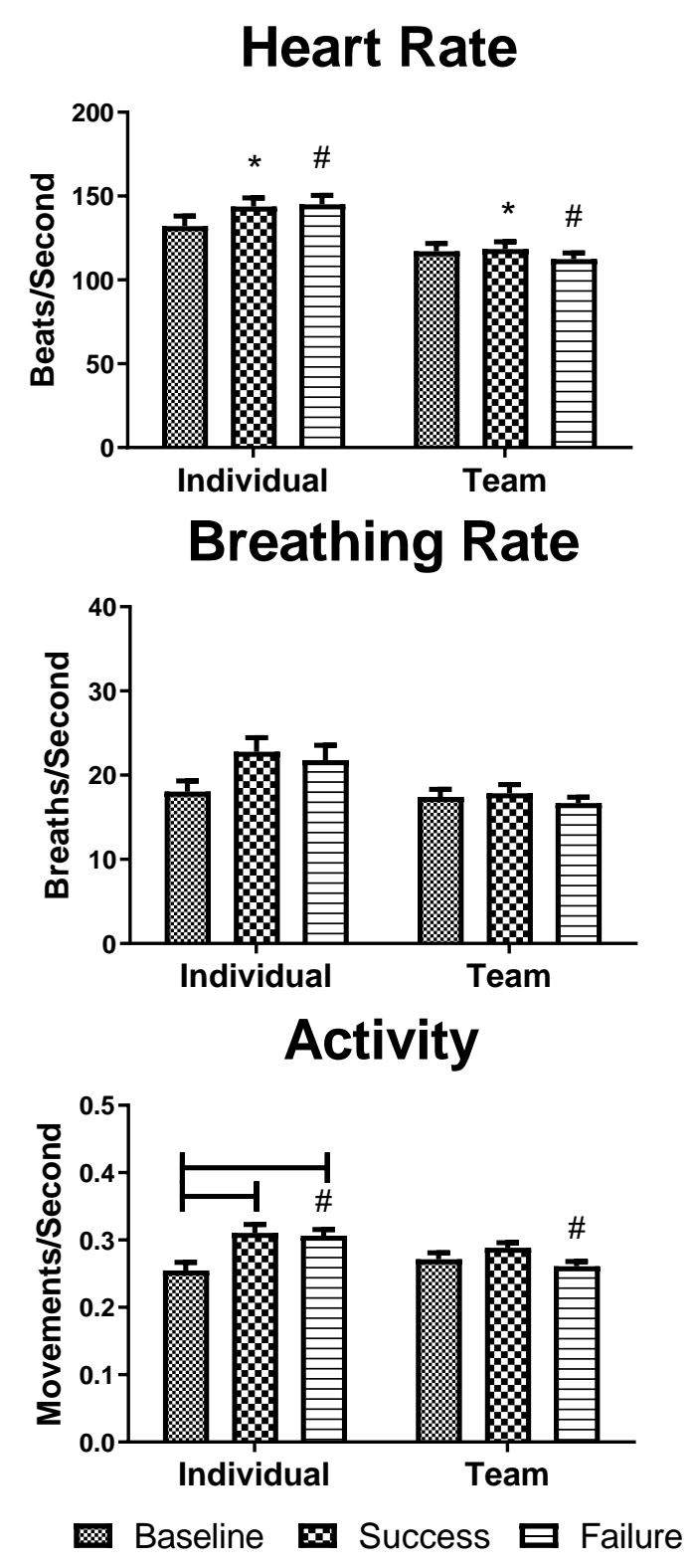

Figure 2.1. Physical activity markers of stress in a mock rescue mission. Physical activity was measured for individuals $(\mathrm{n}=15)$ while completing a mission as individuals and part of a team using external Zephyr ${ }^{\mathrm{TM}}$ sensors. Physical activity markers were calculated using the summed activity (generated by Zephyr ${ }^{\mathrm{TM}}$ ) normalized by the time (seconds) it took to complete the mission as described in Materials and Methods. The physical activity metric was averaged for all 
individuals. Significance levels were determined using the Friedman test with a Dunn's post-test, where \# and * indicate significance $(\mathrm{p}<0.05)$ between an individual and team mission. Bars indicate significance $(\mathrm{p}<0.05)$ within individual or team missions. Data are represented as mean \pm SEM.

\subsubsection{Individual salivary cytokine response to success and failure}

Cytokine concentrations were measured in saliva when the subjects first arrived (premission) and ten minutes post completion of each mission (baseline, success, and failure). All eight cytokines displayed significant increases $(\mathrm{p}<0.05)$ following the failed mission compared to success (Figure 2.2) determined by the Friedman test with Dunn's post-test. Conversely, a significant decrease in cytokine response was observed following the successful mission compared to baseline for IL-6 and TNF- $\alpha$ (Figure 2.2). 


\section{Individual}

\section{IL-10}

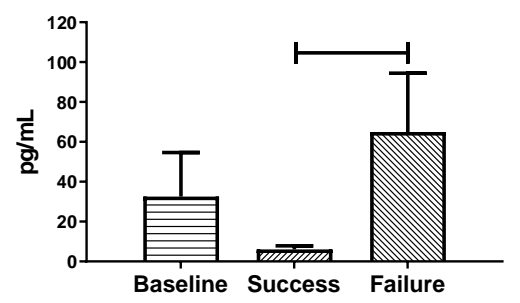

IL-5

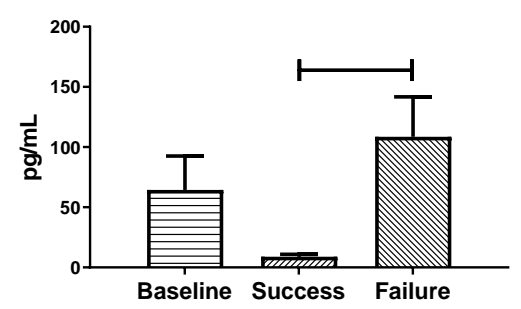

IL-4

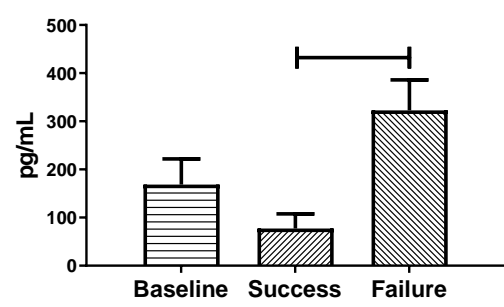

TNF-a

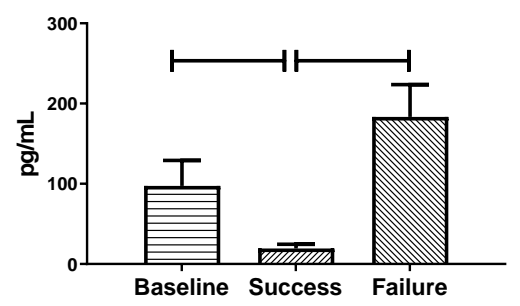

GM-CSF

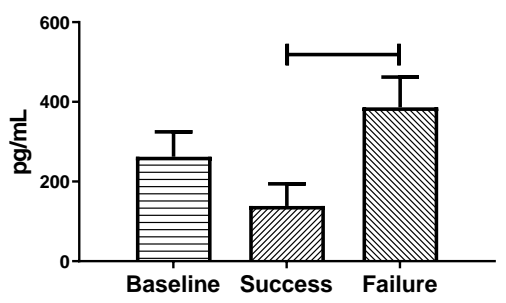

IFN-y

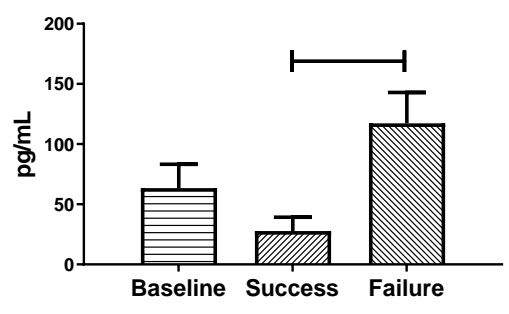

IL-2

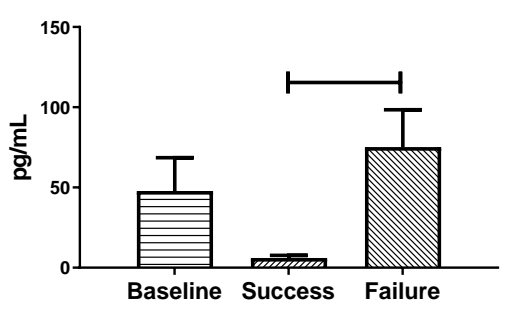

IL-6

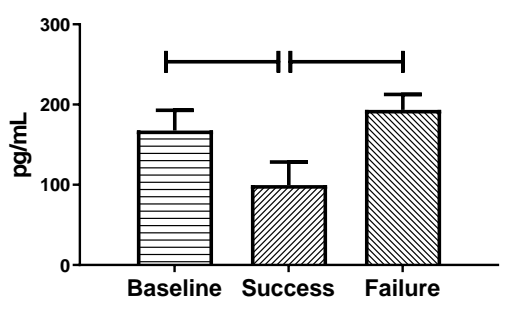

Figure 2.2. Salivary cytokine concentrations following individual missions. Cytokines were measured for individuals $(\mathrm{n}=15)$ while completing a mission as individuals. Cytokine responses (IL-10, IL-6, GM-CSF, IL-5, IFN- $\gamma$, TNF- $\alpha$, IL-2, IL-4) were measured in saliva after each mission using a multiplex ELISA as described in Materials and Methods. Significance levels were determined using the Friedman test with a Dunn's post-test. Bars indicate significant differences $(\mathrm{p}<0.05)$ between missions. Data are represented as mean \pm SEM. 


\subsubsection{Team salivary cytokine responses to success and failure}

Cytokine concentrations were also measured in saliva after the team missions. While performing as a team, none of the cytokines displayed significant differences between baseline, success, and failure (Figure 2.3). However, the baseline cytokine response for individuals on the day of the team mission were significantly increased $(\mathrm{p}<0.05)$ compared to the day of individual completion determined by Wilcoxon matched-pairs signed ranked test (Figure 2.4). 


\section{Team}

IL-10

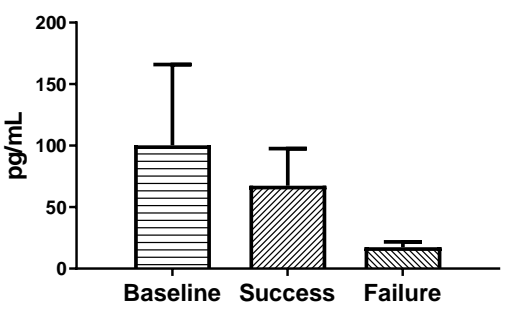

IL-5

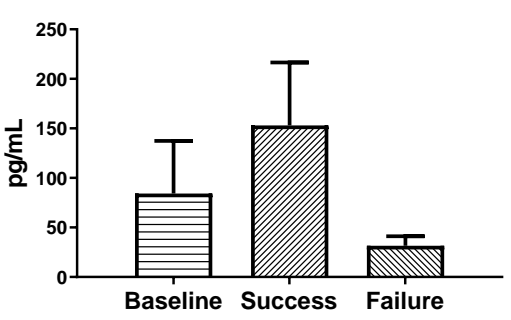

IL-4

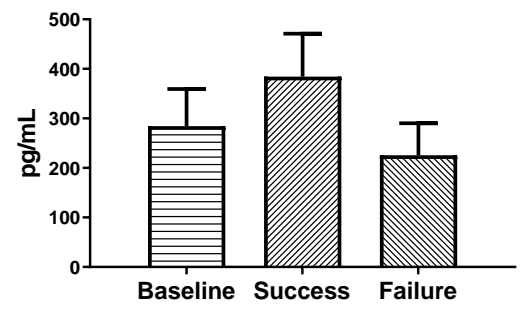

TNF-a

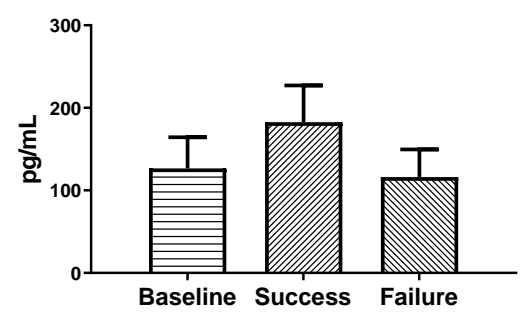

GM-CSF

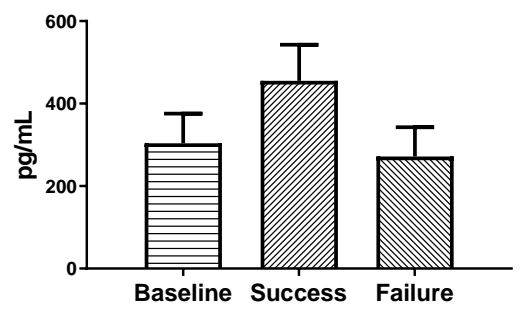

IFN-y

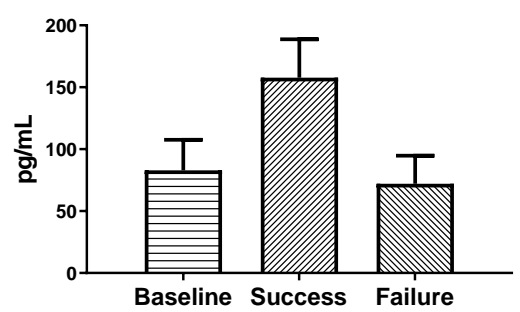

IL-2

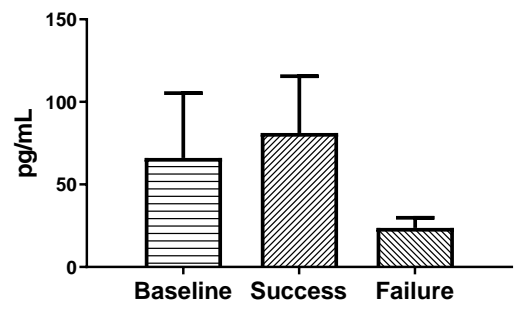

IL-6

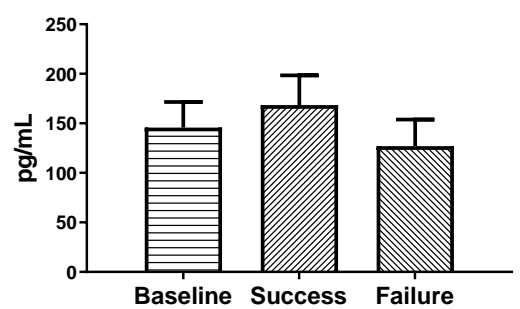

Figure 2.3. Salivary cytokine concentrations following team missions. Cytokines were measured for individuals $(\mathrm{n}=15)$ while completing a mission on teams. Cytokine responses (IL-10, IL-6, GM-CSF, IL-5, IFN- $\gamma$, TNF- $\alpha$, IL-2, IL-4) were again measured in saliva after each mission. There were no significant differences ( $p>0.05)$ as determined by the Friedman test with a Dunn's post-test. Data are represented as mean \pm SEM. 


\section{Pre-Mission}
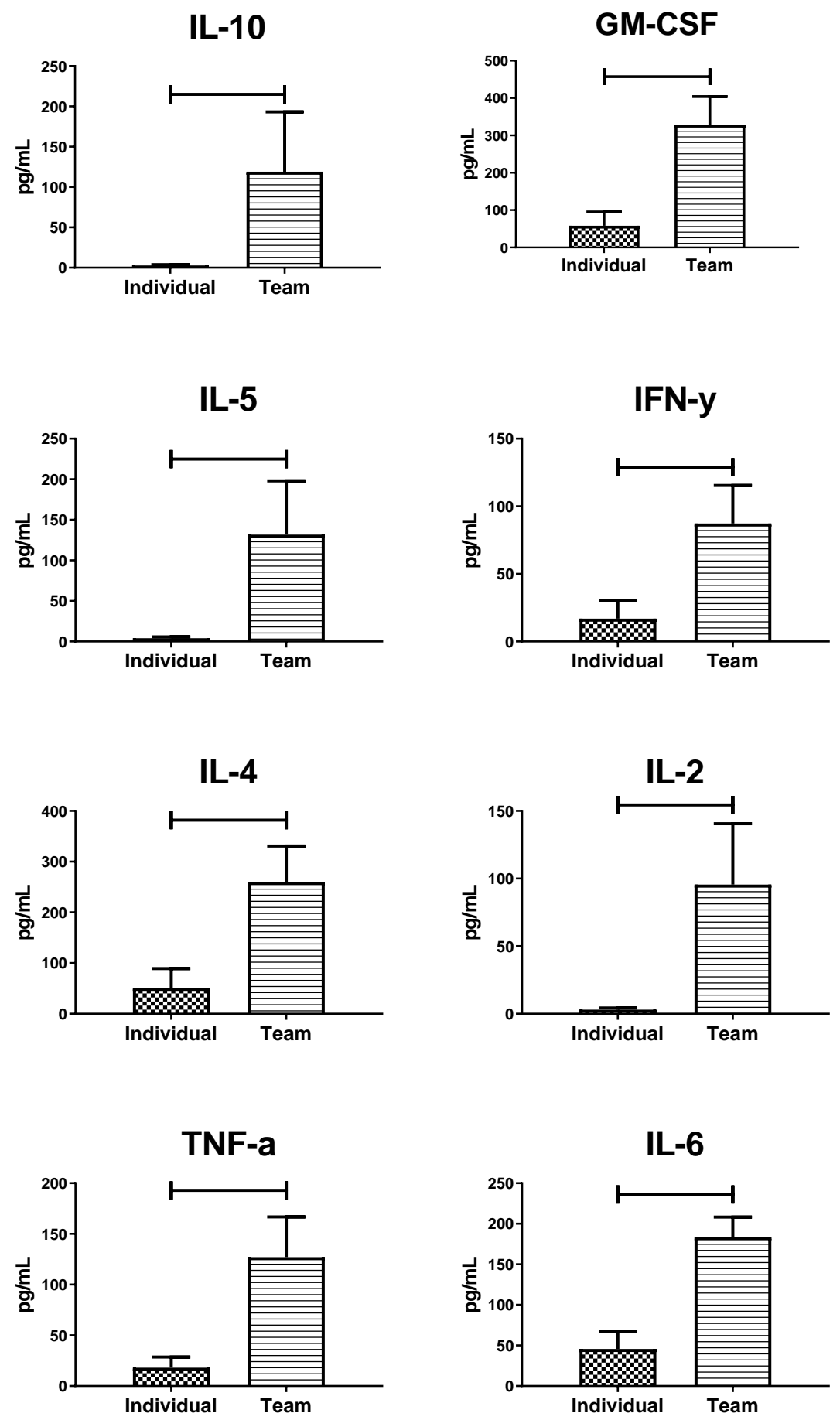

Figure 2.4. Cytokine responses pre-mock rescue missions. Cytokines were measured for individuals $(\mathrm{n}=15)$ before completing the missions as individuals and as teams. Significance levels were determined using the Friedman test 
with a Dunn's post-test, where bars indicate significant differences $(\mathrm{p}<0.05)$ between individual and team baseline. Data are represented as mean \pm SEM.

\subsection{Discussion}

Social ties are highly important for survival, and thus threats to our social connections (e.g., rejection, isolation, conflict, or loss) signal to our body that one is more vulnerable and may face a greater likelihood of wounding and infection and, as such, a greater need for inflammatory activity marked by increased cytokine concentrations is needed [25]. Within this study, Air Force ROTC cadets were enrolled to perform a mock hostage rescue mission and cytokine responses were measured in combination with physical activity to better understand the individual stress response to success and failure in high stress field situations.

The Zephyr ${ }^{\mathrm{TM}}$ bioharness device provides reliable and valid measurements that can be continuously taken and monitored while completing activities [26]. The wearable device provides various physical activity parameters, such as heart rate, breathing rate, and activity levels, which are important in monitoring physical intensity during exercises. Within our study, there were no significant differences in physical load as determined by heart rate, breathing rate, and activity levels between success and failure while completing the missions as individuals or in a team setting (Figure 2.1). However, we did observe an increased response for individuals for success and failure from baseline for activity/time. Due to the fact that participants were instructed to complete tasks as quickly as possible during the successful and failed missions, it was expected that the subjects would move more efficiently during these two tasks. Additionally, there was a decreased response for the averaged team heart rate and activity from the individual metrics, which is expected due to the shared physical load while completing the mission in teams.

Salivary biomarker analysis provides a unique opportunity to collect saliva multiple times on the same day, without significant limitations. As such, within this study, cytokine levels were 
able to be monitored following completion of each mission. After a failed mission, cytokine levels were found to be significantly increased for all of the measured cytokines compared to success (Figure 2.2). These increased responses of failure may be reflective of a defeat resulting in psychological stress or anxiety [27-28]. In addition to the 2004 study, Dickerson et al. (2009) demonstrated that individuals who performed the Trier Social Stress Test in front of an evaluative panel showed increases in proinflammatory cytokines from pre- to post-stress compared to those individuals who performed the same task, but completed it alone (eliminating the social stress) [29]. Likewise, in our experimental setup, the individuals returned to the rendezvous point where all the investigators and ROTC commanders were waiting. Thus, these increased cytokines may be in response to the social defeat experienced in front of a group. Conversely, successful missions resulted in decreased cytokine levels compared to baseline for IL-6 and TNF- $\alpha$ (Figure 2.2). This lack of inflammatory response is likely a feeling of relief or acceptance within the social setting after performing well.

Pearson correlation analyses were completed to determine if any significant correlations existed between physical activity metrics and cytokine levels within each mission. There were no significant correlations (data not shown) for any of the individual missions to the physical activity data. These results indicate the cytokine responses observed for individual failure and success for the subjects undergoing a mock rescue mission were independent of physical stress.

To further understand if the cytokine responses were a result of social or mental stress, a repeat study was performed on the same individuals in a team environment. Neither success nor failure caused a significant difference while performing as a team (Figure 2.3). Thus, in the team setting, the same response to success and failure is not experienced, but they were still experiencing 
the same mental stimulation. These results indicate that the increased inflammatory response is not a result of mental stress.

Interestingly, the pre-mission cytokine levels measured on the day of completion for individuals in a team were significantly higher than on the day of individual mission (Figure 2.4). The human body, especially the endocrine, immune, and cardiovascular systems, is exceedingly sensitive and responsive to social interactions [30]. In this study, cytokine responses were immediately heightened in a team setting compared to an individual setting without any physical or mental stimulation. Thus, the increased immune response to the team setting further indicates the elevated cytokine levels in response to failure for individuals are in response to social stressors.

\subsection{Conclusions}

In summary, using a noninvasive biomonitoring technique, this study was able to determine cytokine levels in response to success and failure. Furthermore, individuals experienced higher cytokines in response to failure and to a team setting. Additionally, the individual cytokine responses did not correlate to physical load as measured by a $\mathrm{Zephyr}^{\mathrm{TM}}$ bioharness. These cytokines could be important to better understand social stress during a physical exercise, especially for military personnel (and possibly could be extended to sports teams in the future).

Importantly, this study was conducted in a naturalistic setting for these personnel and followed subjects through three distinct scenarios (baseline, success, and failure). It should be noted that one limitation of this study was small sample size (15 subjects). However, the sample size does meet statistical power analysis standards, and future research will aim to increase the size of the teams to explore larger groups. While these results indicate a noninvasive method of determining social stress levels under challenging conditions, this relationship should be further investigated to better understand potential differences in cytokine levels among genders. 


\subsection{Acknowledgements}

The authors wish to thank all of the participants involved in the study for their time and dedication. The authors would also like to acknowledge those that helped in the study: the West Virginia University Air Force ROTC commanders for helping us recruit participants, Dr. Matthew Loos for helping collect samples, and Constance Mitchell for helping set up the crime scene house and collecting samples. This study was funded by the Defense Advanced Research Projects Agency (DARPA) and NIH IDeA Networks of Biomedical Research Excellence (INBRE) (W911NF-12-0165). 


\subsection{References}

1. La Fratta, I., Tatangelo, R., Campagna, G., R. et al. (2018) The plasmatic and salivary levels of IL-1 $\beta$, IL-18 and IL-6 are associated to emotional difference during stress in young male. Sci. Rep., 8(1), 3031. DOI: 10.1038/s41598-018-21474-y

2. Segerstrom, S. C., \& Miller, G. E. (2004) Psychological stress and the human immune system: a meta-analytic study of 30 years of inquiry. Psychol. Bull., 130(4), 601-630. DOI: 10.1037/00332909.130.4.601

3. Cohen, S., Janicki-Deverts, D., Doyle, W. J., et al. (2012). Chronic stress, glucocorticoid receptor resistance, inflammation, and disease risk. Proc. Natl. Acad. Sci. U.S.A., 109(16), 59955999. DOI: $10.1073 /$ pnas. 1118355109

4. McEwen, B. (2005) Stressed and stressed out: What is the difference? J. Psychiatry Neurosci., 30(5), 315-318.

5. McEwen, B. (2007) Physiology and neurobiology of stress and adaptation: Central role of the brain. Physiol. Rev., 87(3), 873-904. DOI: 10.1152/physrev.00041.2006

6. Chida, Y., Hamer, M., Wardle, J., \& Steptoe, A. (2008) Do stress-related psychosocial factors contribute to cancer incidence and survival? Nat. Clin. Pract. Oncol., 5(8), 466-475. DOI: $10.1038 /$ ncponc 1134

7. Sapolsky, R. M., Romero, L. M., \& Munck, A. U. (2000) How do glucocorticoids influence stress responses? Integrating permissive, suppressive, stimulatory, and preparative actions. Endocr. Rev., 21(1), 55-89. DOI: 10.1210/edrv.21.1.0389

8. Nunes, L.A., Mussavira, S. \& Bindhu, O.S. (2015) Clinical and diagnostic utility of saliva as a non-invasive diagnostic fluid: a systematic review. Biochem. Med., 25(2), 177-192. DOI: 10.11613/BM.2015.018

9. Papacoasta, E. \& Nassis, G.P. (2011) Saliva as a tool for monitoring steroid, peptide and immune markers in sport and exercise science. J. Sci. Med. Sport, 14(5), 424-434. DOI: 10.1016/j.jsams.2011.03.004

10. Nater, U.M. \& Rohleder, N. (2009) Salivary alpha-amylase as a non-invasive biomarker for the sympathetic nervous system: current state of research. Psychoneuroendocrinology, 34(4), 486496. DOI: 10.1016/j.psyneuen.2009.01.014

11. Nunes, L.A., Brenzikofer, R. \& Macedo, D.V. (2011) Reference intervals for saliva analytes collected by a standardized method in a physically active population. Clin. Biochem., 44(17-18), 1440-1444. DOI: 10.1016/j.clinbiochem.2011.09.012

12. Duque, G.A. \& Descoteaux, A. (2014) Macrophage Cytokines: Involvement in Immunity and Infectious Diseases. Front. Immunol., 5, 491. DOI: 10.3389/fimmu.2014.00491 
13. Prasad, S., Tyagi, A.K. \& Aggarwal, B.B. (2016) Detection of inflammatory biomarkers in saliva and urine: Potential in diagnosis, prevention, and treatment for chronic diseases. Exp. Biol. Med., 241(8), 783-799. DOI: 10.1177/1535370216638770

14. Moldoveanu, A., Shephard, R.J. \& Shek, P.N. (2001) The cytokine response to physical activity and training. Sports Med., 31(2), 115-144. DOI: 10.2165/00007256-200131020-00004

15. Slavish, D.C., Graham-Engeland, J.E., Smyth, J.M. \& Engeland, C.G. (2015) Salivary markers of inflammation in response to acute stress. Brain Behav. Immun., 44, 253-269. DOI: 10.1016/j.bbi.2014.08.008

16. Chiang, J.J., Eisenberger, N.I., Seeman, T.E. \& Taylor, S.E. (2012) Negative and competitive social interactions are related to heightened proinflammatory cytokine activity. Proc. Natl. Acad. Sci. U.S.A., 109(6), 1878-1882. DOI: 10.1073/pnas.1120972109

17. Dickerson, S. \& Kemeny, M. (2004a) Acute stressors and cortisol responses: a theoretical integration and synthesis of laboratory research. Psychol. Bull., 130(3), 355-391. DOI: 10.1037/0033-2909.130.3.355

18. Dickerson, S.S., Kemeny, M.E., Aziz, N., Kim, K.H. \& Fahey, J.L. (2004b). Immunological effects of induced shame and guilt. Psychosom. Med., 66(1), 124-131. DOI: 10.1097/01.psy.0000097338.75454.29

19. Dowlati, Y., Herrmann, N., Swardfager, W., et al. (2010) A meta-analysis of cytokines in major depression. Biol. Psychiatry, 67(5), 446-457. DOI: 10.1016/j.biopsych.2009.09.033

20. Denollet, J., Vrints, C.J. \& Conraads, V.M. (2008) Comparing Type D personality and older age as correlates of tumor necrosis factor-alpha dysregulation in chronic heart failure. Brain Behav. Immun., 22(5), 736-743. DOI: 10.1016/j.bbi.2007.10.015

21. Audet, M.C., Jacobson-Pick, S., Wann, B.P. \& Anisman, H. (2011a) Social defeat promotes specific cytokine variations within the prefrontal cortex upon subsequent aggressive or endotoxin challenges. Brain Behav. Immun., 25(6), 1197-1205. DOI: 10.1016/j.bbi.2011.03.010

22. Stanton, S.J. \& Schultheiss, O.C. (2010). The hormonal correlates of implicit power motivation. J. Res. Pers., 43(5), 942. DOI: 10.1016/j.jrp.2009.04.001

23. Wirth, M.M., Welsh, K.M. \& Schultheiss, O.C. (2006) Salivary cortisol changes in humans after winning or losing a dominance contest depend on implicit power motivation. Horm. Behav., 49(3), 346-352. DOI: 10.1016/j.yhbeh.2005.08.013

24. Zephyr Technology. (2012) BioHarness 3.0 User Manual. https://www.zephyranywhere.com/media/download/bioharness3-user-manual.pdf (accessed December 2, 2019).

25. Eisenberger, N., Moieni, M., Inagaki, T.K., Muscatell, K.A. \& Irwin, M.R. (2017) In Sickness and in Health: The Co-Regulation of Inflammation and Social Behavior. Neuropsychopharmacology, 42(1), 242-253. DOI: 10.1038/npp.2016.141 
26. Nazari, G., Bobos, P., MacDermid, J.C., et al. (2018) Psychometric properties of the Zephyr bioharness device: a systematic review. BMC Sports Sci. Med. Rehabil., 10, 6. DOI: 10.1186/s13102-018-0094-4

27. Audet, M.C., Mangano, E.N., \& Anisman, H. (2011b) Behavior and pro-inflammatory cytokine variations among submissive and dominant mice engaged in aggressive encounters: moderation by corticosterone reactivity. Front. Behav. Neurosci., 4(156), 1-12. DOI: 10.3389/fnbeh.2010.00156

28. Takahashi, A., Flanigan, M.E., McEwen, B.S. \& Russo, S.J. (2018) Aggression, Social Stress, and the Immune System in Humans and Animal Models. Front. Behav. Neurosci., 12, 56. DOI: 10.3389/fnbeh.2018.00056

29. Dickerson, S.S., Gable, S.L., Irwin, M.R. Aziz, N. \& Kemeny, M.E. (2009). Social-evaluative threat and proinflammatory cytokine regulation an experimental laboratory investigation. Psychol. Sci., 20(10), 1237-1244. DOI: 10.1111/j.1467-9280.2009.02437.x

30. Heaphy, E., \& Dutton, J. (2008) Positive social interactions and the human body at work: Linking organizations and physiology. Academy of Management Review, 33(1), 137-162. DOI: $10.2307 / 20159380$ 


\section{Chapter 3: Corticosterone ameliorates diisopropyl fluorophosphate-induced MAPK phosphoprotein signaling in a mouse brain}

Each year, millions of people are exposed to organophosphates (OPs) which may lead to nerve agent induced seizures and irreversible brain injury. Even those that survive acute OP exposure report continued symptoms of adverse neurological effects years later. Previous highexposure OP research has shown the activation of MAPK signaling pathways depending on the type of OP and brain region, but the focus of this research has been on OPs in isolation. Certain occupations (e.g., military, farmers, and pest control specialists) are more vulnerable to high dose OP exposure and are also susceptible to high levels of occupation-related stress. To address a more realistic mixture exposure, corticosterone (CORT) has been used to stimulate a response associated with high stress that would likely be simultaneously experienced during OP exposures. Previous results have shown this combination increases neuroinflammation, but ameliorates acetylcholine in discrete brain regions. As a follow-up to these experiments, an investigation into phosphoprotein responses in discrete brain regions was performed to better understand the early cellular changes. Using a mouse model, adult male C57BL/6J mice were exposed to CORT $(200 \mu \mathrm{g} / \mathrm{mL})$ in the drinking water for seven days followed by a single injection of diisopropyl fluorophosphate (DFP; $4.0 \mathrm{mg} / \mathrm{kg}$, i.p.) on day eight and euthanized $0.5,2$, and $24 \mathrm{~h}$ post-exposure. To evaluate brainregion-specific effects, 14 post-translationally modified protein targets were measured using multiplexed enzyme-labelled immunosorbent assay (ELISA) beads for the cortex and hippocampus. Brain region-specific phosphoprotein responses were found to significantly differ from exposure to DFP alone (p-AKT, p-BAD, p-JNK, and p-SYK) and when combined with preexposure to CORT (p-SRC). Specifically, the phosphoprotein responses to DFP at $24 \mathrm{~h}$ activates 
phosphoproteins involved in mitogen-activated protein kinase (MAPK) signaling; however, when pre-exposed to CORT these results were ameliorated. These results display the short-term protective ability CORT may provide to OP exposure, but could relate to the delayed onset of neurological effects.

\subsection{Introduction}

Organophosphate (OP) toxicity can result from household to occupational exposures (e.g., field workers, pesticide sprayers, military, etc.). The primary mode of action for OPs is inhibition of acetylcholinesterase (AChE) by binding to the enzyme at the active serine site, which results in cholinergic toxicity due to the persistent stimulation of the cholinergic receptors [1]. Coinciding with AChE inhibition, acute and chronic exposure to OPs has shown to increase pro-inflammatory cytokines, which in turn leads to neuroinflammation [2]. While many animal studies have been conducted to better understand OP toxicity in isolation, relatively few studies have addressed the impacts of stress on the toxicity of these OP nerve agents.

Those at an increased risk of high OP exposure (e.g., agriculture workers and military personnel) have also been reported as some of the most stressful occupations [3-7]. Therefore, it is important to study the combined effects of OP exposure and stress. To better reflect this exposure, O'Callaghan et al. (2015), established a mouse model using the combination of an OP, diisopropyl fluorophosphate (DFP), coupled with corticosterone (CORT; the rodent surrogate for cortisol) pretreatment at levels that would be associated with high stress [8]. The pre-treatment with CORT enhanced neuroinflammation, assessed by qPCR of several pro-inflammatory cytokines (tumor necrosis factor- $\alpha$, interleukin $1 \beta$ and 6 ), within the first six hours in the frontal cortex and hippocampus when compared to DFP alone [8]. Interestingly, Locker et al. demonstrated when 
using this model that priming with CORT "recovered" AChE activity that was inhibited by DFP alone [9], indicating the observed neuroinflammation may be independent of AChE inhibition.

Cytokine and AChE signaling, similar to those observed in this combinatory model, can directly initiate cellular signaling changes in impacted tissues, which can be measured through post-translational modifications (e.g., protein phosphorylation) at early time points post-exposure [8-11]. Specifically, this includes the family of mitogen-activated protein kinases (MAPKs) that are crucial in the cellular responses to cytokines and implicated in immune and neuroinflammatory diseases [12-14]. Similarly, phosphoinositide 3-kinases (PI3Ks) are key in regulating several mechanisms in the inflammatory response to external insults, and the dysregulation of this pathway has been shown to be involved in multiple neurological diseases [15-16]. Therefore, an investigation examining the phosphorylation of proteins, both upstream and downstream of these pathways, within the first $24 \mathrm{~h}$ in a mouse brain post-exposure to CORT, DFP, and the combination was undertaken. The proteins measured in this study, highlight the relationship between regulatory stress and inflammatory pathways associated with early responses of neuroinflammation.

In the study presented here, adult male C57BL/6J mice were exposed to CORT (200 $\mu \mathrm{g} / \mathrm{mL})$ in the drinking water for seven days, and, on the eighth day, were given a single intraperitoneal (i.p.) injection of saline or DFP (4.0 mg/kg), based on the O'Callaghan et al. model (2015) [8]. Mice were euthanized $0.5,2$, and $24 \mathrm{~h}$ post-exposure via focused microwave irradiation to preserve the steady-state levels of protein phosphorylation [17]. To elucidate the effects of cellular signal transduction post-exposure to CORT, DFP, and a CORT+DFP mixture on discrete brain regions, 14 post-translationally modified protein targets were measured in the cortex and hippocampus. This approach provides insight to the acute phosphoprotein signaling observed in CORT+OP exposure based on the elucidation of relevant spatiotemporal phosphorylation responses. 


\subsection{Materials and methods}

\subsubsection{Materials}

The following drugs and chemicals were obtained from the sources indicated: CORT (Steraloids Inc., Newport, RI, USA), DFP (Sigma-Aldrich Co. St. Louis, MO, USA), ethanol (Sigma-Aldrich), phenylmethylsulfonyl fluoride (Sigma-Aldrich), and cell lysis buffer (Bio-Rad, Hercules, CA, USA). A Pierce BCA assay was obtained from Fisher Scientific (Pittsburgh, PA, USA) and includes albumin standards and working reagent. A custom Bio-Plex Pro multiplexed magnetic bead-based immunoassay kit was purchased from Bio-Rad. The kit included capture antibodies, biotinylated secondary antibody, standard diluents, streptavidin-phycoerythrin, resuspension buffer, wash buffer, cell lysis buffer, and positive and negative controls.

\subsubsection{Animals}

Adult male C57BL/6J mice (approximately 8-12 weeks of age) were purchased from Jackson Laboratory (Bar Harbor, ME, USA). All procedures were performed under protocols approved by the Institutional Animal Care and Use Committee of the Centers for Disease Control and Prevention, National Institute for Occupational Safety and Health, and the animal facility was certified by the American Association for Accreditation of Laboratory Animal Care. Upon arrival, the mice were individually housed in a temperature- $\left(21 \pm 1^{\circ} \mathrm{C}\right)$ and humidity-controlled $(50 \pm$ $10 \%$ ) colony room that was maintained under filtered positive-pressure ventilation on a $12 \mathrm{~h}$ light (06:00 hours)/12 h dark cycle. The plastic cages were 46 x 25 x $15 \mathrm{~cm}$; cage bedding consisted of heat-treated pine shavings at a depth of $4 \mathrm{~cm}$. Mice were given ad libitum access to food and water.

\subsubsection{Dosing}

Mice (at least five/group; animals were arbitrarily assigned to groups by the experimenter) were exposed to CORT and DFP as previously described [8]. Briefly, mice received CORT in the 
drinking water (200 $\mu \mathrm{g} / \mathrm{mL}$ in $1.2 \%$ ethanol) for seven days prior to exposure (Figure 3.1). Control groups received $1.2 \%$ ethanol water. At this exposure, CORT was found to be elevated, similar to those who would be experiencing high stress levels and has been shown to accentuate, or "prime", the central nervous system to produce a robust proinflammatory response to neurotoxicants or inflammogens [19]. On day eight, mice were given a single intraperitoneal (i.p.) injection of saline $(0.9 \%)$ or DFP $(4.0 \mathrm{mg} / \mathrm{kg})$ in the morning and returned to their home cage (experimenter was not blinded). This exposure of DFP elicited seizure-like behaviors within minutes post-injection [19].

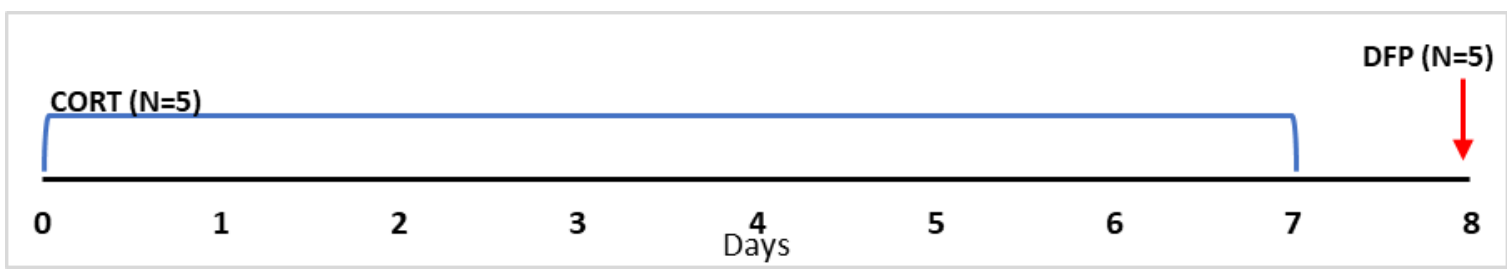

Figure 3.1. Dosing paradigm for corticosterone and diisopropyl fluorophosphate exposure. Timeline for administration of diisopropyl fluorophosphate (DFP $4.0 \mathrm{mg} / \mathrm{kg}$ ) after corticosterone (CORT) priming (200 $\mu \mathrm{g} / \mathrm{mL}$ in 1.2\% ethanol) in drinking water.

\subsubsection{Brain dissection}

Mice were euthanized at three time points $(0.5,2$, and $24 \mathrm{~h}$ post-exposure) by focused microwave irradiation. By using focused microwave irradiation for euthanasia, changes to the steady-state levels of protein phosphorylation in response to exposures can be preserved in the brain to provide a snapshot of the early cell signaling alterations that result in long-term impacts using a high-throughput approach [17]. Whole brains were removed from the skull and dissected free-hand with fine curved forceps on a thermoelectric cold plate (Model TCP-2; Aldrich Chemical Co., Milwaukee, WI, USA) into two brain regions: cortex and hippocampus. Samples were immediately snap frozen and stored at $-80^{\circ} \mathrm{C}$ until analysis. Mice that did not survive to the end points were excluded from analysis. 


\subsubsection{Protein quantification in brain regions}

Protein extraction was performed using methods adapted from Hulse et al. (2004) [20]. One side of the brain region (e.g., left cortex) was transferred to a $2 \mathrm{~mL}$ tube, and $500 \mu \mathrm{L}$ of cell lysis buffer containing $2 \mathrm{mM}$ phenylmethylsulfonyl fluoride was added. Samples were homogenized using a sonic dismembrator (model 120; Fisher Scientific) on ice with 3 rapid pulses for 3 seconds at 35\% amplitude until tissue was sufficiently ground (about 5 times). The samples were centrifuged at $4500 \mathrm{x} \mathrm{g}$ for $10 \mathrm{~min}$ at $4{ }^{\circ} \mathrm{C}$, and the supernatant was collected and stored at $-80^{\circ} \mathrm{C}$ until analysis. Total protein concentration was determined using the Pierce BCA assay in duplicate, according to the manufacturer's instructions. Briefly, albumin standards were generated ranging in concentration from $0-2000 \mu \mathrm{g} / \mathrm{mL}$ in sample diluent. Each standard or unknown was pipetted into a microplate well, and $200 \mu \mathrm{L}$ of the working reagent was added. The plate was incubated at $37^{\circ} \mathrm{C}$ for $0.5 \mathrm{~h}$. Absorbance values were determined at $562 \mathrm{~nm}$ using an Infinite M1000 plate reader (Tecan, Raleigh, NC).

\subsubsection{Phosphoprotein analysis in discrete brain regions}

For phosphoprotein analysis, sample homogenates were diluted to a total protein concentration of $700 \mu \mathrm{g} / \mathrm{mL}$ with sample diluent and centrifuged at $15000 \mathrm{x} \mathrm{g}$ for $10 \mathrm{~min}$ at $4{ }^{\circ} \mathrm{C}$. Samples were assayed using the Bio-Plex Pro multiplexed magnetic bead-based immunoassay reagent kit for the following targets phosphorylated at the indicated amino acid residues: p-AKT (S473), p-BAD (S136), p-S6 Ribosomal P (S235/S236; p-RPS6), p-SMAD2 (S465/S467), p-SRC (Y416), p-SYK (Y352), p-CREB (S133), p-ERK 1/2 (T202/Y204, T185/Y187), p-GSK3 $\alpha / \beta$

(S21/S9), p-HSP27 (S78), p-IкB $\alpha$ (S32/S36), p-JNK (T183/Y185), p-MEK 1/2 (S217/S221), and p-p53 (S15). Samples were analyzed using a Bio-Plex 200 suspension array system and Pro II Wash Station (Bio-Rad), according to the manufacturer's instructions. Briefly, Bio-Plex magnetic 
bead-based assays follow a typical sandwich enzyme-linked immunosorbent assay (ELISA) protocol. Capture antibodies are covalently coupled to the beads and react overnight with analytes in the sample. After several washes, a biotinylated detection antibody is added and incubated for $0.5 \mathrm{~h}$. After another series of washes, the detection complex (streptavidin-phycoerythrin) is added and incubated for 10 minutes. After a final series of washes, a resuspension buffer is added and data are acquired on the Bio-Plex 200. Biological replicates were at least in quintuplicate, and assay measurements were performed in duplicate (technical replicates). Bio-Rad lysate positive (EGF-treated HEK-293, UV-treated HEK-293, H2O2-treated Ramos, EGF-treated SK-BR-3, TNF- $\alpha$-treated HeLa, and PDGF-treated NIH3T3) and negative (phosphatase-treated HeLa) controls were used to ensure appropriate bead counts.

\subsubsection{Statistical analysis}

Power calculations were preformed using SAS JMP (V14; Cary, NC, USA) and was determined to be four mice per group using previous standard deviations, a power of 0.8 , and $\alpha=0.05$. No exclusion criteria were pre-determined.

Relative phosphorylation was determined for each target. The averaged raw values were blank-subtracted, and any phosphoprotein non-detects (response was less than blank) were removed before analysis. The blank-subtracted values were normalized to saline control at each time point for each brain region. A Grubbs' test was performed to remove any significant outliers $(\alpha=0.05)$. As previously mentioned, any non-surviving animals were also excluded from analysis. Thus, these analyses resulted in at least $\mathrm{N}=4$ animals for the CORT, DFP, and CORT+DFP groups for all phosphoproteins in both brain regions. Data analyses were performed using GraphPad Prism (V5; San Diego, CA, USA). The normalized phosphorylation \pm the standard error of the mean (SEM) for each exposure and time point were used to identify significant targets. Two-way 
analysis of variance (ANOVA) with Bonferroni post-test was used to determine significance using GraphPad Prism. A significance level of $5 \%(\mathrm{p}<0.05)$ was considered statistically significant.

\subsection{Results}

The cortex and hippocampus were extracted from C57BL/6J mice following exposure to saline control, CORT alone, DFP alone, and a combination of CORT and DFP. A panel of phosphoproteins involved in regulatory stress and inflammatory pathways associated with early responses of neuroinflammation were assayed from brain region samples via multiplex bead-based ELISA. The phosphoprotein responses were normalized to saline controls in the cortex and hippocampus at $0.5,2$, and $24 \mathrm{~h}$ post-exposure. While all of the saline, CORT alone, and CORT+DFP mice survived at the $24 \mathrm{~h}$ time point, only 5 of 14 mice from the DFP exposure alone group survived (36\% survival rate). The increased mortality associated with DFP has been noted by other researchers at this exposure level who have previously used this model [8, 21-23]. However, the activated signaling pathways from the surviving mice may reveal important biomarkers with relevance to symptoms associated with acute OP exposure. Specific patterns observed spatially and temporally are discussed over the following sections.

\subsubsection{Relative phosphorylation responses in the cortex}

Within the cortex, there were several phosphoproteins that were significantly different $(\mathrm{p}<0.05)$ compared to other exposures in response to DFP alone exposure. Phosphorylated RPS6 and SMAD2 were significantly increased $(\mathrm{p}<0.05)$ at $0.5 \mathrm{~h}$ for DFP relative to CORT+DFP (Figure 3.2). At $2 \mathrm{~h}, \mathrm{p}$-RPS6 and p-HSP27 were also significantly increased $(\mathrm{p}<0.05)$ post-DFP exposure relative to saline control (Figure 3.2). A significant increase $(\mathrm{p}<0.05)$ for $\mathrm{p}$-JNK at $24 \mathrm{~h}$ postexposure to DFP alone compared to the other exposures was also observed (Figure 3.2). Conversely, DFP alone caused a significant decrease $(\mathrm{p}<0.05)$ in phosphorylation for AKT at 24 
h (Figure 3.2) relative to CORT+DFP. Additionally, the combination of CORT+DFP resulted in a significant decrease $(\mathrm{p}<0.05)$ in $\mathrm{p}-\mathrm{SRC}$ at $24 \mathrm{~h}$ compared to all other exposures (Figure 3.2). Phosphorylated HSP27 also was significantly increased $(\mathrm{p}<0.05)$ for CORT alone relative to saline at $2 \mathrm{~h}$ (Figure 3.2).

\section{CORTEX}
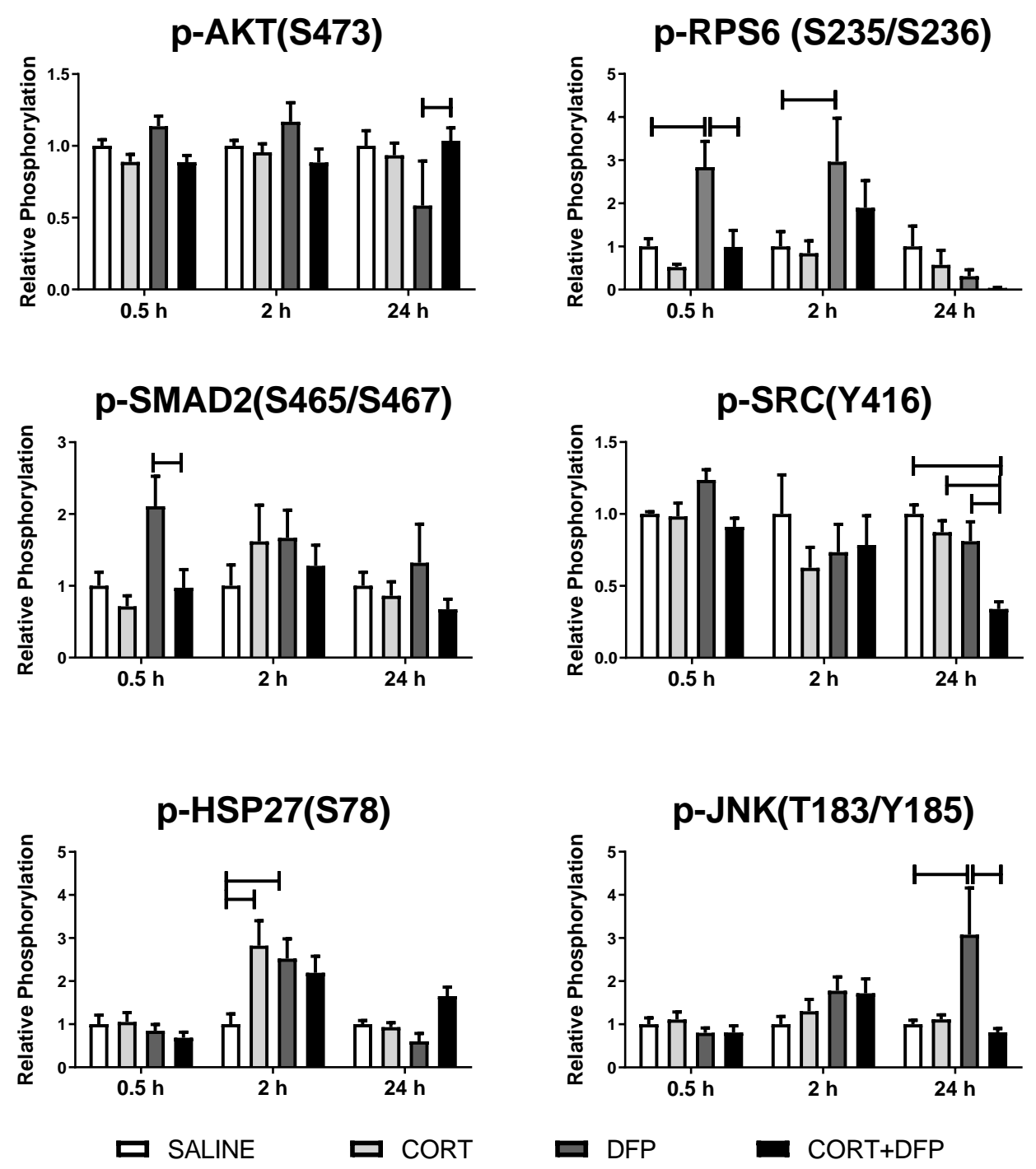

Figure 3.2. Significant phosphoprotein responses in the cortex via multiplex ELISA post-exposure to corticosterone and diisopropyl fluorophosphate. Mice were exposed to control or $200 \mu \mathrm{g} / \mathrm{mL}$ CORT in drinking water for seven days (Figure 3.1). On day eight, mice were given DFP (4.0 mg/kg, i.p.) or saline and euthanized via focused microwave 
irradiation at $0.5,2$, or $24 \mathrm{~h}$ post-exposure. Phosphoprotein responses were measured in the cortex after DFP exposure using a multiplex ELISA for targets of interest, as described in Materials and Methods. Significance was determined using two-way ANOVA with Bonferroni post-test, where the horizontal bars represent significance $\mathrm{p}<0.05$ between exposures within a specific time. Data are represented as mean \pm SEM.

Temporally, there was a significant decrease $(\mathrm{p}<0.05)$ in phosphoprotein responses at $24 \mathrm{~h}$ post-exposure compared to the early time points $(0.5 \mathrm{~h}$ and $2 \mathrm{~h})$ post-DFP alone exposure for $\mathrm{p}$ AKT, p-RPS6, p-CREB, and p-p53 (Table 3.1). Conversely, p-JNK was significantly increased $(\mathrm{p}<0.05)$ at $24 \mathrm{~h}$ compared to the early time points for DFP alone. At $2 \mathrm{~h}, \mathrm{p}$-ERK1/2 and p-RPS6 were significantly increased $(\mathrm{p}<0.05)$ for CORT+DFP compared to $0.5 \mathrm{~h}$ and $24 \mathrm{~h}$, respectively (Table 3.1). Phosphorylated p53 was also significantly increased $(\mathrm{p}<0.05)$ at the $2 \mathrm{~h}$ time point for CORT alone. Interestingly, HSP27 was significantly phosphorylated $(\mathrm{p}<0.05)$ at the $2 \mathrm{~h}$ time point for all exposures.

Table 3.1. Significant temporal phosphoprotein responses in the cortex post-exposure to corticosterone and diisopropyl fluorophosphate. Data are represented as mean $\pm \mathrm{SEM}$ with at least $\mathrm{N}=4$ for all phosphoproteins. Twoway ANOVA with Bonferroni post-test was used for determining significance, where * indicates a significant difference $(\mathrm{p}<0.05)$ between 0.5 h vs. $2 \mathrm{~h}$, \# indicates a significant difference $(\mathrm{p}<0.05)$ between $0.5 \mathrm{~h}$ vs. $24 \mathrm{~h}$, and \% indicates a significant difference $(\mathrm{p}<0.05)$ between $2 \mathrm{~h}$ vs. $24 \mathrm{~h}$ for a particular exposure.

\begin{tabular}{|l|l|l|l|l|l|}
\hline \multirow{4}{*}{ Phosphoprotein } & Exposure & $0.5 \mathrm{~h}$ & $2 \mathrm{~h}$ & $24 \mathrm{~h}$ & \multirow{2}{*}{ Time (h) } \\
\hline \multirow{3}{*}{ p-AKT (S473) } & Cort & $0.89 \pm 0.05$ & $0.96 \pm 0.06$ & $0.93 \pm 0.09$ & \\
\cline { 2 - 6 } & DFP & $1.14 \pm 0.07$ & $1.17 \pm 0.13$ & $0.58 \pm 0.31$ & \#,\% \\
\cline { 2 - 6 } & Cort+DFP & $0.89 \pm 0.05$ & $0.88 \pm 0.09$ & $1.04 \pm 0.09$ & \\
\hline \multirow{3}{*}{ p-RPS6 (S235/S236) } & Cort & $0.52 \pm 0.07$ & $0.85 \pm 0.29$ & $0.57 \pm 0.34$ & \\
\cline { 2 - 6 } & DFP & $2.84 \pm 0.60$ & $2.96 \pm 1.01$ & $0.31 \pm 0.15$ & $\#, \%$ \\
\cline { 2 - 6 } & Cort+DFP & $0.99 \pm 0.38$ & $1.90 \pm 0.63$ & $0.04 \pm 0.01$ & $\%$ \\
\hline p-SRC (Y416) & Cort & $0.98 \pm 0.09$ & $0.63 \pm 0.14$ & $0.87 \pm 0.08$ & \\
\hline
\end{tabular}




\begin{tabular}{|c|c|c|c|c|c|}
\hline & DFP & $1.24 \pm 0.07$ & $0.73 \pm 0.19$ & $0.81 \pm 0.13$ & $*$ \\
\hline & Cort+DFP & $0.91 \pm 0.06$ & $0.78 \pm 0.20$ & $0.34 \pm 0.05$ & $\#$ \\
\hline \multirow{3}{*}{ p-CREB (S133) } & Cort & $1.76 \pm 0.64$ & $2.25 \pm 0.44$ & $0.83 \pm 0.16$ & \\
\hline & DFP & $1.41 \pm 0.62$ & $2.20 \pm 1.03$ & $0.16 \pm 0.08$ & $\%$ \\
\hline & Cort+DFP & $1.18 \pm 0.39$ & $2.67 \pm 0.47$ & $0.85 \pm 0.21$ & \\
\hline \multirow{3}{*}{$\begin{array}{l}\text { p-ERK1/2 } \\
\text { (T202/Y204, } \\
\text { T185/Y187) }\end{array}$} & Cort & $1.35 \pm 0.27$ & $2.17 \pm 0.73$ & $1.06 \pm 0.20$ & \\
\hline & DFP & $0.89 \pm 0.24$ & $1.71 \pm 0.66$ & $0.94 \pm 0.32$ & \\
\hline & Cort+DFP & $0.66 \pm 0.17$ & $2.63 \pm 0.87$ & $1.27 \pm 0.23$ & $*$ \\
\hline \multirow{3}{*}{ p-HSP27 (S78) } & Cort & $1.05 \pm 0.22$ & $2.82 \pm 0.58$ & $0.93 \pm 0.11$ & $*, \%$ \\
\hline & DFP & $0.85 \pm 0.15$ & $2.52 \pm 0.46$ & $0.60 \pm 0.19$ & $*, \%$ \\
\hline & Cort+DFP & $0.68 \pm 0.13$ & $2.20 \pm 0.38$ & $1.65 \pm 0.21$ & $*, \#$ \\
\hline \multirow{3}{*}{ p-JNK (T183/Y185) } & Cort & $1.11 \pm 0.17$ & $1.30 \pm 0.28$ & $1.11 \pm 0.11$ & \\
\hline & DFP & $0.80 \pm 0.11$ & $1.78 \pm 0.32$ & $3.08 \pm 1.09$ & $\#, \%$ \\
\hline & Cort+DFP & $0.81 \pm 0.15$ & $1.72 \pm 0.33$ & $0.82 \pm 0.09$ & \\
\hline \multirow{3}{*}{ p-p53 (S15) } & Cort & $1.15 \pm 0.21$ & $1.67 \pm 0.51$ & $0.77 \pm 0.05$ & $\%$ \\
\hline & DFP & $0.85 \pm 0.12$ & $1.28 \pm 0.21$ & $0.45 \pm 0.16$ & $\%$ \\
\hline & Cort+DFP & $0.99 \pm 0.18$ & $1.30 \pm 0.27$ & $0.86 \pm 0.09$ & \\
\hline
\end{tabular}

\subsubsection{Relative phosphorylation responses in the hippocampus}

In the hippocampus, several phosphoproteins were again observed to have significant differences $(\mathrm{p}<0.05)$ involving DFP at 2 and $24 \mathrm{~h}$ post-exposure. Similar to the cortex, p-SYK and p-JNK were significantly increased $(\mathrm{p}<0.05)$ for DFP alone compared to CORT+DFP at $24 \mathrm{~h}$, while p-RPS6 and p-SMAD2 were increased relative to saline $(\mathrm{p}<0.05)$ at $2 \mathrm{~h}$ (Figure 3.3). Additionally, p-MEK1/2, p-ERK1/2, and p-CREB were also significantly phosphorylated $(\mathrm{p}<0.05)$ at $24 \mathrm{~h}$ for DFP compared to saline or CORT+DFP. Conversely, at $24 \mathrm{~h}, \mathrm{p}-\mathrm{AKT}$ was significantly decreased $(\mathrm{p}<0.05)$ for DFP compared to CORT+DFP. Phosphorylated I $\mathrm{B} \alpha$ and SMAD2 were significantly increased $(\mathrm{p}<0.05)$ for CORT-alone compared to saline at $0.5 \mathrm{~h}$ and $2 \mathrm{~h}$, respectively 
(Figure 3.3). At $0.5 \mathrm{~h}, \mathrm{p}-\mathrm{CREB}$ was significantly increased $(\mathrm{p}<0.05)$ for CORT+DFP compared to CORT-alone (Figure 3.3). Interestingly, p-BAD was significantly increased at $24 \mathrm{~h}$ for CORT+DFP relative to CORT and DFP alone (Figure 3.3). 


\section{Hippocampus}
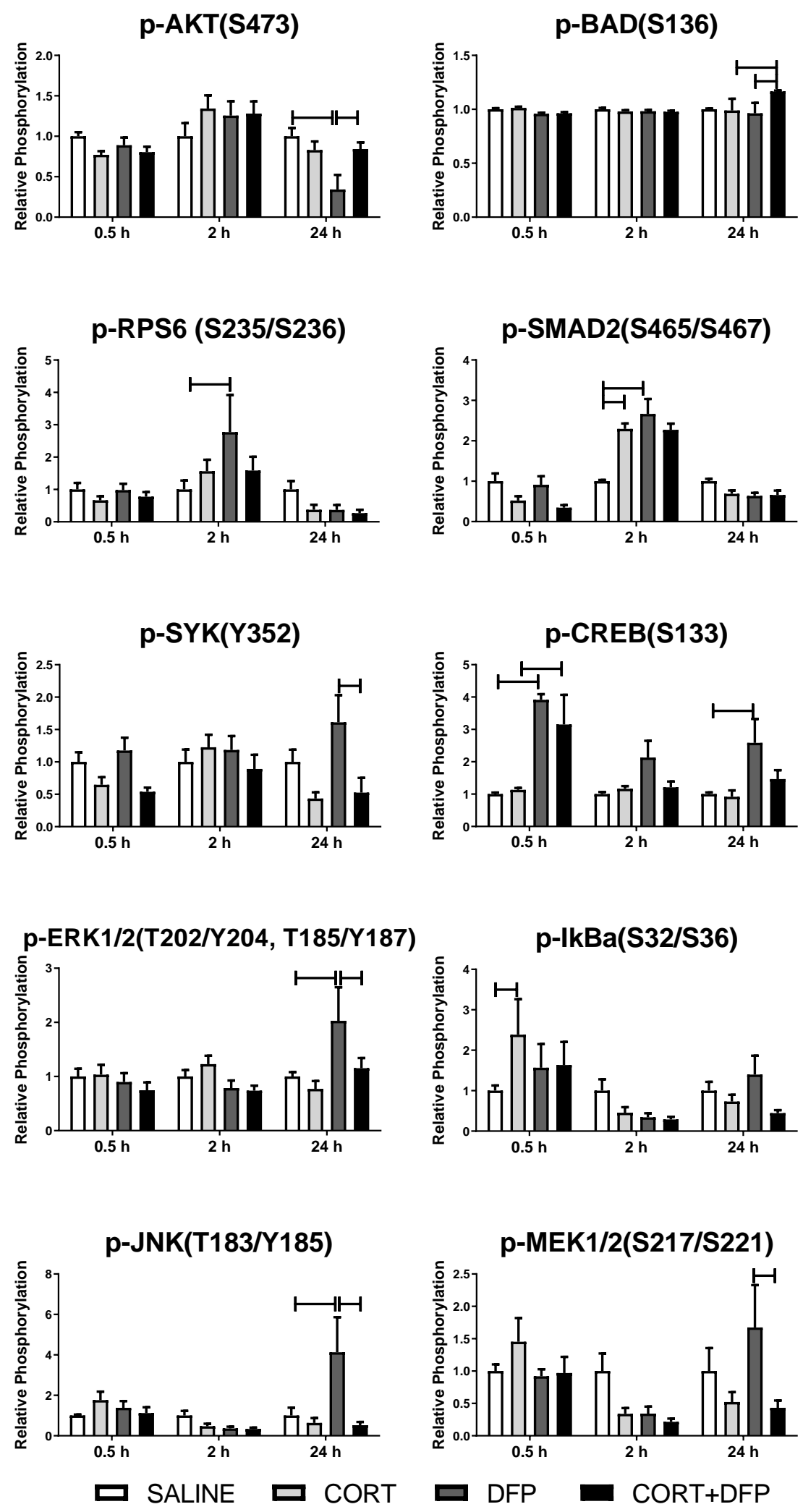
Figure 3.3. Significant phosphoprotein responses in the hippocampus via multiplex ELISA post-exposure to corticosterone and diisopropyl fluorophosphate. Using the same dosing regimen and euthanasia as stated in Figure 3.2, phosphoprotein responses in the hippocampus were measured using multiplex ELISA for targets of interest. Two-way ANOVA with Bonferroni post-test was used for determining significance, where the horizontal bars represent significance $\mathrm{p}<0.05$ between exposures within a specific time. Data are represented as mean \pm SEM.

Temporally, there was at least one significant difference $(p<0.05)$ in each of the phosphoproteins tested, with the exception of p-p53 (Table 3.2). DFP alone significantly phosphorylated $(\mathrm{p}<0.05) \mathrm{HSP} 27$ and CREB at $0.5 \mathrm{~h}$, while at $2 \mathrm{~h}, \mathrm{p}-\mathrm{SMAD} 2$ and p-RPS6 were significantly increased. DFP alone significantly decreased $(\mathrm{p}<0.05) \mathrm{p}-\mathrm{AKT}$ and $\mathrm{p}-\mathrm{SRC}$ at $24 \mathrm{~h}$ post-exposure. Alternatively, p-ERK1/2, p-GSK3 $\alpha / \beta$, p-JNK, and p-MEK1/2 were all significantly increased $(\mathrm{p}<0.05)$ following DFP exposure at $24 \mathrm{~h}$ relative to the earlier time points (Table 3.2). At $24 \mathrm{~h}, \mathrm{p}-\mathrm{BAD}$ was also significantly increased $(\mathrm{p}<0.05)$ compared to the earlier time points, but following CORT+DFP exposure. Phosphorylated CREB was significantly increased $(\mathrm{p}<0.05)$ at $0.5 \mathrm{~h}$ relative to 2 and $24 \mathrm{~h}$ post-exposure for CORT+DFP. For p-AKT and p-SMAD2, CORT alone and CORT+DFP caused significant increases $(\mathrm{p}<0.05)$ at $2 \mathrm{~h}$ compared to the other time points. CORT alone also resulted in a significant increase $(\mathrm{p}<0.05)$ at $2 \mathrm{~h}$ for $\mathrm{p}-\mathrm{SRC}$ and $\mathrm{p}-\mathrm{SYK}$, while $\mathrm{p}-\mathrm{I} \kappa \mathrm{B} \alpha$ and $\mathrm{p}-\mathrm{MEK} 1 / 2$ were significantly increased $(\mathrm{p}<0.05)$ at $0.5 \mathrm{~h}$.

Table 3.2. Significant temporal phosphoprotein responses in the hippocampus post-exposure to corticosterone and diisopropyl fluorophosphate. Data are represented as mean \pm SEM with at least $\mathrm{N}=4$ for all phosphoproteins in the table. Two-way ANOVA with Bonferroni post-test was used for determining significance, where * indicates a significant difference $(\mathrm{p}<0.05)$ between 0.5 h vs. 2 h, \# indicates a significant difference $(p<0.05)$ between 0.5 h vs. $24 \mathrm{~h}$, and \% indicates a significant difference $(\mathrm{p}<0.05)$ between $2 \mathrm{~h}$ vs. $24 \mathrm{~h}$ for a particular exposure.

\begin{tabular}{|l|l|l|l|l|l|}
\hline & & \multicolumn{3}{|l|}{ Time (h) } & \\
\cline { 3 - 5 } Phosphoprotein & Exposure & $0.5 \mathrm{~h}$ & $2 \mathrm{~h}$ & $24 \mathrm{~h}$ & Significant \\
\hline p-AKT (S473) & Cort & $0.77 \pm 0.04$ & $1.34 \pm 0.16$ & $0.83 \pm 0.11$ & $*, \%$ \\
\hline
\end{tabular}




\begin{tabular}{|c|c|c|c|c|c|}
\hline & DFP & $0.89 \pm 0.10$ & $1.26 \pm 0.18$ & $0.34 \pm 0.18$ & $\#, \%$ \\
\hline & Cort+DFP & $0.80 \pm 0.07$ & $1.28 \pm 0.15$ & $0.84 \pm 0.08$ & $*, \%$ \\
\hline \multirow{3}{*}{ p-BAD (S136) } & Cort & $1.01 \pm 0.01$ & $0.98 \pm 0.01$ & $0.99 \pm 0.11$ & \\
\hline & DFP & $0.96 \pm 0.01$ & $0.98 \pm 0.01$ & $0.96 \pm 0.10$ & \\
\hline & Cort+DFP & $0.96 \pm 0.01$ & $0.98 \pm 0.01$ & $1.17 \pm 0.01$ & $\#, \%$ \\
\hline \multirow{3}{*}{ p-RPS6 (S235/S236) } & Cort & $0.67 \pm 0.12$ & $1.56 \pm 0.35$ & $0.37 \pm 0.15$ & \\
\hline & DFP & $0.98 \pm 0.19$ & $2.77 \pm 1.14$ & $0.37 \pm 0.15$ & $*, \%$ \\
\hline & Cort+DFP & $0.78 \pm 0.14$ & $1.58 \pm 0.43$ & $0.27 \pm 0.10$ & \\
\hline \multirow{3}{*}{$\begin{array}{l}\text { p-SMAD2 } \\
\text { (S465/S467) }\end{array}$} & Cort & $0.52 \pm 0.11$ & $2.29 \pm 0.13$ & $0.69 \pm 0.08$ & $*, \%$ \\
\hline & DFP & $0.91 \pm 0.21$ & $2.66 \pm 0.37$ & $0.64 \pm 0.07$ & $*, \%$ \\
\hline & Cort+DFP & $0.35 \pm 0.06$ & $2.27 \pm 0.15$ & $0.66 \pm 0.11$ & $*, \%$ \\
\hline \multirow{3}{*}{ p-SRC (Y416) } & Cort & $0.83 \pm 0.24$ & $2.37 \pm 0.60$ & $0.28 \pm 0.18$ & $\%$ \\
\hline & DFP & $1.60 \pm 0.54$ & $2.24 \pm 1.16$ & $0.10 \pm 0.05$ & $\%$ \\
\hline & Cort+DFP & $1.28 \pm 0.52$ & $1.42 \pm 0.51$ & $0.17 \pm 0.09$ & \\
\hline \multirow{3}{*}{ p-SYK (Y352) } & Cort & $0.65 \pm 0.12$ & $1.23 \pm 0.19$ & $0.43 \pm 0.10$ & $\%$ \\
\hline & DFP & $1.18 \pm 0.20$ & $1.18 \pm 0.22$ & $1.61 \pm 0.42$ & \\
\hline & Cort+DFP & $0.54 \pm 0.06$ & $0.89 \pm 0.22$ & $0.53 \pm 0.23$ & \\
\hline \multirow{3}{*}{ p-CREB (S133) } & Cort & $1.13 \pm 0.06$ & $1.17 \pm 0.08$ & $0.92 \pm 0.20$ & \\
\hline & DFP & $3.92 \pm 0.17$ & $2.13 \pm 0.52$ & $2.58 \pm 0.74$ & $*$ \\
\hline & Cort+DFP & $3.15 \pm 0.92$ & $1.21 \pm 0.18$ & $1.46 \pm 0.28$ & $*, \#$ \\
\hline \multirow{3}{*}{$\begin{array}{l}\text { p-ERK1/2 } \\
\text { (T202/Y204, } \\
\text { T185/Y187) }\end{array}$} & Cort & $1.03 \pm 0.18$ & $1.23 \pm 0.15$ & $0.77 \pm 0.15$ & \\
\hline & DFP & $0.90 \pm 0.16$ & $0.79 \pm 0.14$ & $2.03 \pm 0.62$ & $\#, \%$ \\
\hline & Cort+DFP & $0.75 \pm 0.15$ & $0.74 \pm 0.09$ & $1.15 \pm 0.19$ & \\
\hline \multirow{3}{*}{$\mathrm{p}-\mathrm{GSK} 3 \alpha / \beta(\mathrm{S} 21 / \mathrm{S} 9)$} & Cort & $0.80 \pm 0.07$ & $0.78 \pm 0.13$ & $0.72 \pm 0.13$ & \\
\hline & DFP & $0.70 \pm 0.09$ & $0.74 \pm 0.11$ & $1.34 \pm 0.38$ & \# \\
\hline & Cort+DFP & $0.72 \pm 0.16$ & $0.55 \pm 0.06$ & $0.80 \pm 0.17$ & \\
\hline \multirow{3}{*}{ p-HSP27 (S78) } & Cort & $1.01 \pm 0.17$ & $0.37 \pm 0.16$ & $0.46 \pm 0.29$ & \\
\hline & DFP & $1.19 \pm 0.21$ & $0.42 \pm 0.18$ & $0.39 \pm 0.11$ & $*, \#$ \\
\hline & Cort+DFP & $0.94 \pm 0.28$ & $0.33 \pm 0.10$ & $0.33 \pm 0.13$ & \\
\hline
\end{tabular}




\begin{tabular}{|l|l|l|l|l|l|}
\cline { 2 - 6 } & Cort & $2.39 \pm 0.87$ & $0.45 \pm 0.14$ & $0.73 \pm 0.17$ & $*, \#$ \\
\cline { 2 - 7 } $\mathrm{p}-\mathrm{I} \kappa \mathrm{B} \alpha(\mathrm{S} 32 / \mathrm{S} 36)$ & $\mathrm{DFP}$ & $1.57 \pm 0.59$ & $0.34 \pm 0.10$ & $1.40 \pm 0.47$ & \\
\cline { 2 - 7 } & Cort+DFP & $1.63 \pm 0.57$ & $0.29 \pm 0.07$ & $0.45 \pm 0.07$ & \\
\hline \multirow{3}{*}{$\mathrm{p}-\mathrm{JNK}(\mathrm{T} 183 / \mathrm{Y} 185)$} & Cort & $1.77 \pm 0.42$ & $0.47 \pm 0.13$ & $0.64 \pm 0.24$ & \\
\cline { 2 - 7 } & DFP & $1.38 \pm 0.34$ & $0.36 \pm 0.08$ & $4.13 \pm 1.73$ & $\#, \%$ \\
\cline { 2 - 7 } & Cort+DFP & $1.12 \pm 0.29$ & $0.34 \pm 0.07$ & $0.53 \pm 0.16$ & \\
\hline \multirow{3}{*}{$\begin{array}{l}\text { p-MEK1/2 } \\
\text { (S217/S221 })\end{array}$} & Cort & $1.45 \pm 0.37$ & $0.34 \pm 0.09$ & $0.52 \pm 0.15$ & $*$ \\
\cline { 2 - 7 } & DFP & $0.92 \pm 0.11$ & $0.34 \pm 0.11$ & $1.67 \pm 0.65$ & $\%$ \\
\cline { 2 - 7 } & Cort+DFP & $0.97 \pm 0.25$ & $0.22 \pm 0.05$ & $0.43 \pm 0.11$ & \\
\hline
\end{tabular}

\subsection{Discussion}

Each year, millions of people are exposed to OPs, and approximately ten percent of exposures result in death, but even those that survive high-dose exposures report symptoms of adverse neurological effects for years later [2]. Moreover, certain occupations that at an increased risk for high-dose OP exposure are also most likely to have increased stress levels [3-8]. In order to better understand this realistic exposure in these vulnerable groups, several researchers have conducted animal studies using exogenous CORT, to replicate high organismal stress, in combination with DFP, an irreversible OP AChE inhibitor [8, 24]. These experiments have been informative showing increased and chronic neuroinflammation compared to DFP exposure alone [8]. Conversely, inhibition of brain AChE activity by DFP appears to be ameliorated by prior CORT exposure, suggesting a potential AChE-independent route to cause this neuroinflammatory response [9, 25]. Thus, an investigation into early intracellular signaling responses via phosphoprotein analysis in discrete brain regions was performed within this study to understand the responses that may lead to these AChE-independent route of a chronic disease state. 
Previous research has highlighted the differences in the spatiotemporal progression of intoxication of DFP and showed detrimental effects of exposure occur in the cortex first (typically in the first $6 \mathrm{~h}$ ), while lesions in the hippocampus are delayed until 12-24 h post-exposure [26-27]. The results in the present study agree, displaying delays in phosphoprotein responses in the hippocampus relative to the cortex. Specifically, p-RPS6 and p-SMAD2 are significantly increased $(\mathrm{p}<0.05)$ at $0.5 \mathrm{~h}$ post-DFP exposure in the cortex, but are not increased in the hippocampus until $2 \mathrm{~h}$ (Figures 3.2, 3.3). Additionally, SRC phosphorylation in the cortex begins to decrease after 0.5 $\mathrm{h}$ for all exposures, but is increased at $2 \mathrm{~h}$ post-exposure for the hippocampus and then decreased by $24 \mathrm{~h}$. Therefore, it is essential to observe the early cellular spatiotemporal responses that involve post-translational phosphorylation events and regulate adaptive stress and inflammatory pathways as these converge toward a new cellular- and ultimately organism-level steady state [28]. Elucidation of the spatiotemporal phosphorylation responses in combination with previous research on neuronal signaling (e.g., neuroinflammatory markers, acetylcholine accumulation) relevant to these exposures holds the potential to further discern the manifestations of continued OP toxicity.

In both brain regions, statistically significant differences $(\mathrm{p}<0.05)$ are observed in phosphoproteins involved in the MAPK signaling pathway in response to DFP alone, which agrees with previous research indicating OPs activate this pathway [29-30]. Two of the main families of MAPKs were measured within this study: ERKs and JNKs. Within this study, DFP alone led to significant increases $(\mathrm{p}<0.05)$ at $24 \mathrm{~h}$ in phosphorylation for $\mathrm{p}-\mathrm{JNK}$ in both brain regions, and $\mathrm{p}$ ERK1/2 and p-MEK1/2 in the hippocampus (Figures 3.2,3.3). Activation of the ERK pathway by growth factors can regulate proliferation, differentiation, and apoptosis via the RAS/RAF/MEK/ERK cascade [31-32]. Conversely, JNKs are activated by environmental 
stressors and inflammatory cytokines (i.e., tumor necrosis factor- $\alpha$, interleukin-1) and plays a key role in regulating apoptosis [33-34]. Similar to the present study, previous OP exposure research, has demonstrated significantly increased levels of phosphorylation of ERKs and JNKs postexposure and induce activation of apoptosis [35-38]. Therefore, the previously observed increases in cytokines may be leading to the activation of p-ERK and p-JNK observed in the present study, which may be resulting in cell death via apoptosis and ultimately lead to the observed animal death.

Our results indicate that DFP alone was also found to impact another key inflammatory pathway, namely PI3Ks. Specifically, phosphorylation of p-AKT and p-BAD were decreased following exposure to DFP alone relative to CORT+DFP. AKT is a pro-survival kinase that inhibits pro-apoptotic activity by phosphorylating $\mathrm{BAD}$ at serine 136 and its sequestering partner 14-3-3 (Figure 3.4) [39]. However, JNK has been shown to phosphorylate 14-3-3, which releases dephosphorylated BAD (Figure 3.4) [40]. From there, BAD targets the mitochondria and promotes apoptosis while antagonizing the effects of AKT signaling [41]. While there are no statistical differences in $\mathrm{p}-\mathrm{BAD}$ in the cortex, this pattern is observed in the hippocampus, in which $\mathrm{p}-\mathrm{BAD}$ is significantly decreased $(\mathrm{p}<0.05)$ following DFP alone compared to CORT+DFP (Figure 3.3). Moreover, $\mathrm{p}$-AKT is observed to be significantly decreased $(\mathrm{p}<0.05)$ in both brain regions following DFP exposure (Figures 3.2, 3.3). Thus, by observing the phosphorylation of these proteins, spatially, our results indicate the differences in activating MAPK and deactivating PI3K signaling for DFP alone relative to CORT+DFP. The overactivation of p-JNK and subsequent decreases in $\mathrm{p}-\mathrm{AKT}$ and $\mathrm{p}-\mathrm{BAD}$ may be the potential cause of the decreased survival rate for the DFP group (36\%).

Phosphorylated SYK was also found to be increased by DFP at $24 \mathrm{~h}$ time points postexposure (Figure 3.3). SYK is involved in multiple biological processes, including adaptive 
immune response and innate immunity; it functions downstream of antigen receptors in immune cells and plays an important role of distinguishing self and non-self [42-43]. Furthermore, SYK has been shown to phosphorylate several downstream pathways including MAPK and PI3K (Figure 3.4) [44-45]. Taken together, this target may explain the pathway associated with the increased activation of $\mathrm{p}-\mathrm{JNK}$ signaling.

While the combination of CORT+DFP ameliorates these significant phosphoprotein responses that were caused by exposure to DFP alone, p-SRC was shown to be statistically significant $(\mathrm{p}<0.05)$ for CORT+DFP across both brain regions (Tables 3.1, 3.2). The SRC family kinases are highly expressed in the central nervous system and control many cellular events such as cell growth, proliferation, and migration [46]. Within our studies, the combination of CORT+DFP consistently causes a decrease in phosphorylated p-SRC (Tables 3.1, 3.2), which has also been demonstrated in rodents after traumatic brain injuries [47-49]. It has been suggested that the decrease in SRC phosphorylation leads to an increase in the degradation and overall loss of Nmethyl-D-aspartate receptors following brain injuries [49-50]. Furthermore, the loss of N-methylD-aspartate receptors has been linked to a number of major nervous system disorders including neurodegenerative diseases, ischemia, pain, and depression [51]. Importantly, the SRC and SYK kinase families usually operate together in signaling pathways involved in innate cell response, with SRC being activated first in response to pathogens, followed by phosphorylation of SYK (Figure 3.4) [52]. Moreover, p-SRC and p-SYK provide interesting biomarkers that unequivocally link neuroimmune responses to previous findings (decreasing $\mathrm{AChE}$ and increasing neuroinflammation post-exposure to DFP when primed with CORT) in this model [8-9, 25].

A summary of these phosphoprotein connections are presented in a network configuration in Figure 3.4 below. While this is a single network, it is critical to note that this figure is attempting 
to represent significant spatiotemporal phosphorylation that are occurring post-exposure at different times and in different brain regions. For example, DFP alone exposure significantly $(\mathrm{p}<0.05)$ phosphorylates SMAD2, RPS6, and CREB at $0.5 \mathrm{~h}$ post-exposure, but at $24 \mathrm{~h}$ there is a shift in phosphorylation; $\mathrm{p}-\mathrm{SYK}$ and $\mathrm{p}-\mathrm{JNK}$ are significantly increased $(\mathrm{p}<0.05)$, while the PI3K and RAS/RAF/MEK/ERK pathways were both decreased. Meanwhile, CORT+DFP exposure at $24 \mathrm{~h}$ resulted in decreases in phosphorylation for SRC, SYK, and JNK, but increases in the PI3K pathway. Similar to DFP alone, the RAS/RAF/MEK/ERK pathway was decreased at $24 \mathrm{~h}$ for CORT+DFP exposure relative to the earlier time points. While limited, this network is an important step to understanding the combination of OP exposure with CORT, which results in early cytokine-driven inflammation. This will ultimately enhance our understanding of brain specific responses that yield neuroinflammation in this model. 


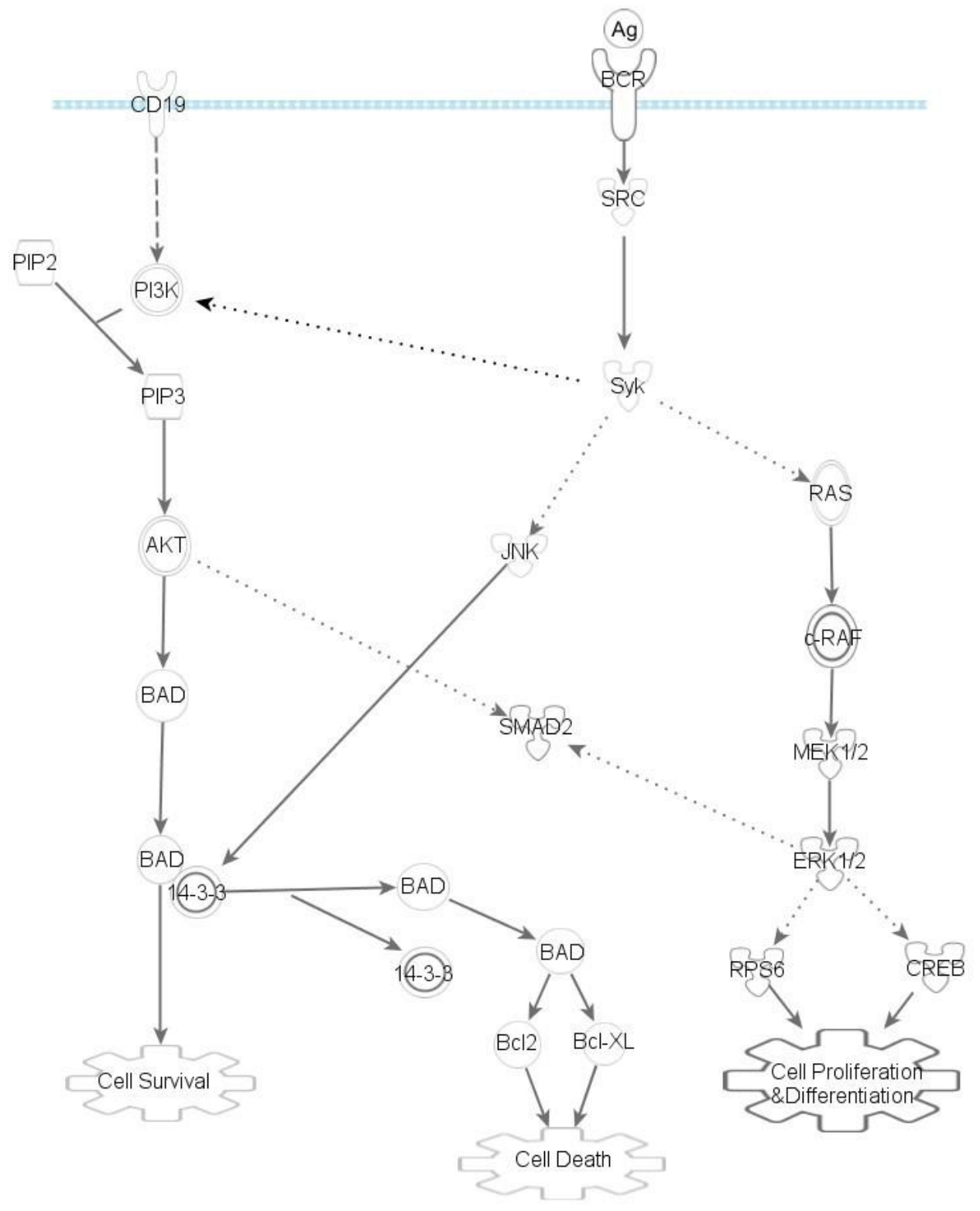

Figure 3.4. Schematic depicting the relationship of SRC with SYK downstream PI3K and MAPK signaling. Binding of the antigen (Ag) to the BCR increases activity of SRC. SYK is then recruited and becomes activated, promoting recruitment and phosphorylation of downstream adaptor or scaffold proteins. Phosphorylation of SYK promotes activation of the MAPK (i.e., JNK), PI3K/AKT, or RAS/RAF/MEK/ERK pathway. The PI3K/AKT pathway promotes phosphorylation of BAD at the serine 136 residue and the association of BAD with 14-3-3, inhibiting pro-apoptotic activity (cell survival). In contrast, JNK phosphorylates 14-3-3 leading to the dissociation of BAD from 14-3-3, a decrease in phosphorylation of $\mathrm{BAD}$, and the dimerization of $\mathrm{BAD}$ with $\mathrm{Bcl}-\mathrm{XL}$ or $\mathrm{Bcl} 2$, eventually resulting in cell 
death. BCR, B-cell receptor; PI3K, phosphoinositide 3-kinase; PIP2, phosphatidylinositol (4,5)-bisphosphate; and PIP3, phosphatidylinositol (3,4,5)-trisphosphate

\subsection{Conclusion}

In summary, DFP-alone exposure causes significant increases in phosphorylation of several proteins involved in the MAPK (p-JNK, p-MEK1/2, p-CREB, p-RPS6) pathway in two brain regions, cortex and hippocampus. Conversely, CORT appears to be partially protective of the inhibition caused by DFP exposure, but leads to significant decreases in p-SRC that corresponds to decreases in p-SYK and p-JNK, but increases in p-AKT and p-BAD. The link between these phosphoprotein targets could be important to further understand how CORT could be involved in activating a currently unknown independent pathway that may lead to survival, but potentially result in delayed OP associated diseases. These phosphoprotein pathways should be further investigated to better understand the long-term protective ability of CORT and the differentiation between the DFP and CORT+DFP exposures. Additionally, future research relating these early intracellular responses to long-term effects of disease progression needs to be performed.

\subsection{Acknowledgements}

The authors gratefully acknowledge James P. O'Callaghan, Kimberly A. Kelly, Lindsay T. Michalovicz, Brenda K. Billig, Christopher M. Felton, Ali Yilmaz and Fang Ma for assistance with dosing the mice and dissecting the brains. The authors would also like to thank Suzanne Danley for her editorial suggestions. This work was supported by Congressionally Directed Medical Research Programs: Gulf War Illness Research Program Grants (GW170081). Opinions, interpretations, conclusions, and recommendations are those of the author and are not necessarily endorsed by the Department of Defense. 


\subsection{References}

1. Fukuto, T. R. (1990) Mechanism of action of organophosphorus and carbamate insecticides. Environ. Health Perspect., 87, 245-254. DOI: 10.1289/ehp.9087245

2. Naughton, S. X., \& Terry, A. V., Jr (2018) Neurotoxicity in acute and repeated organophosphate exposure. Toxicology, 408, 101-112. DOI: 10.1016/j.tox.2018.08.011

3. Pulgar, C. A., Trejo, G., Suerken, C., et al. (2016). Economic Hardship and Depression Among Women in Latino Farmworker Families. Journal of immigrant and minority health, 18(3), 497504. DOI: $10.1007 / \mathrm{s} 10903-015-0229-6$

4. O’Connor, K. Stoecklin-Marois, M., \& Schenker, M.B. (2015) Examining nervios among immigrant male farmworkers in the MICASA Study: sociodemographics, housing conditions and psychosocial factors. J. Immigr. Minor. Health, 17(1), 198-207. DOI: 10.1007/s10903-013-98598

5. Carvajal, S.C., Kibor, C., McClelland, D.J., et al. (2014) Stress and sociocultural factors related to health status among US-Mexico border farmworkers. J. Immigr. Minor. Health, 16(6), 11761182. DOI: $10.1007 / \mathrm{s} 10903-013-9853-1$

6. Bray, R. M., Camlin, C. S., Fairbank, J. A., Dunteman, G. H., \& Wheeless, S. C. (2001). The Effects of Stress on Job Functioning of Military Men and Women. Armed Forces Soc., 27(3), 397417. DOI: $10.1177 / 0095327 \mathrm{X} 0102700304$

7. Harms, P.D., Krasikova, D.V., Vanhove, A.J., Herian, M.N., \& Lester, P.B. (2013) Stress and Emotional Well-Being in Military Organizations. The Role of Emotion and Emotion Regulation in Job Stress and Well Being, 11, 103-132. DOI: 10.1108/S1479-3555(2013)0000011008

8. O'Callaghan, J. P., Kelly, K. A., Locker, A. R., Miller, D. B., \& Lasley S. M. (2015) Corticosterone primes the neuroinflammatory response to DFP in mice: potential animal model of Gulf War Illness. J. Neurochem., 133(5), 708-721. DOI: 10.1111/jnc.13088

9. Locker, A.R., Michalovicz, L.T., Kelly, K.A., et al. (2017) Corticosterone primes the neuroinflammatory response to Gulf War Illness-relevant organophosphates independently of acetylcholinesterase inhibition. J. Neurochem., 142(3), 444-455. DOI: 10.1111/jnc.14071

10. Boyd, J.W. \& Neubig, R. (2019) Cellular Signal Transduction in Toxicology and Pharmacology: Data Collection, Analysis, and Interpretation, John Wiley \& Sons, Ltd, Hoboken.

11. Han, A.A., Currie, H.N., Loos, M.S., et al. (2016) Spatiotemporal phosphoprotein distribution and associated cytokine response of a traumatic injury. Cytokine, 79, 12-22. DOI: 10.1016/j.cyto.2017.10.027

12. Kaminska, B., Gozdz, A., Zawadzka, M., Ellert-Miklaszewska, A. \& Lipko, M. (2009) MAPK signal transduction underlying brain inflammation and gliosis as therapeutic target. Anat. Rec. (Hoboken), 292(12), 1902-1913. DOI: 10.1002/ar.21047 
13. Kotelnikova, E., Kiani, N.A., Messinis, D., et al. (2019) MAPK pathway and B cells overactivation in multiple sclerosis revealed by phosphoproteomics and genomic analysis. Proc. Natl. Acad. Sci. U.S.A., 116(19), 9671-9676. DOI: 10.1073/pnas.1818347116

14. Bedognetti, D., Roelands, J., Decock, J., Wang, E. \& Hendrickx, W. (2017) The MAPK hypothesis: immune-regulatory effects of MAPK-pathway genetic dysregulations and implications for breast cancer immunotherapy. Emerg. Top. Life Sci., 1(5), 429-445. DOI: 10.1042/ETLS20170142

15. Cianciulli, A., Porro, C., Calvello, R. et al. (2020) Microglia mediated neuroinflammation: Focus on PI3K modulation. Biomolecules, 10(1), 137. DOI: 10.3390/biom10010137

16. Zhang, J., Wang, L., Wang, H., Su, Z. \& Pang, X. (2019) Neuroinflammation and central $\mathrm{PI} 3 \mathrm{~K} / \mathrm{Akt} / \mathrm{mTOR}$ signal pathway contribute to bone cancer pain. Mol. Pain, 15, 1744806919830240. DOI: $10.1177 / 1744806919830240$

17. O'Callaghan, J.P., \& Sriram, K. (2004) Focused microwave irradiation of the brain preserves in vivo protein phosphorylation: Comparison with other methods of sacrifice and analysis of multiple phosphoproteins. J. Neurosci. Methods, 135(1-2), 159-168. DOI: 10.1016/j.jneumeth.2003.12.006

18. Kelly, K.A., Michalovicz, L.T., Miller, J.V., et al. (2018) Prior exposure to corticosterone markedly enhances and prolongs the neuroinflammatory response to systemic challenge with LPS. PLoS One, 13(1), e0190546. DOI: 10.1371/journal.pone.0190546

19. Zhu, H., O'Brien, J. J., O'Callaghan, J. P., et al. (2010) Nerve agent exposure elicits site-specific changes in protein phosphorylation in mouse brain. Brain Res., 1342, 11-23. DOI: 10.1016/j.brainres.2010.04.034

20. Hulse, R.E., Kunkler, P.E., Fedynyshyn, J.P., \& Kraig R.P. (2004) Optimization of multiplexed bead-based cytokine immunoassays for rat serum and brain tissue. J. Neurosci. Methods., 136(1), 87-98. DOI: 10.1016/j.jneumeth.2003.12.023

21. Deshpande, L.S., Carter, D.S., Blair, R.E., \& DeLorenzo, R.J. (2010) Development of a Prolonged Calcium Plateau in Hippocampal Neurons in Rats Surviving Status Epilepticus Induced by the Organophosphate Diisopropylfluorophosphate. Toxicol. Sci., 116(2), 623-631. DOI: $10.1093 /$ toxsci/kfq157

22. Tripathi, H.L., \& Dewey, W.L. (1987) Comparison of the effects of diisopropylfluorophosphate, sarin, soman, and tabun on toxicity and brain acetylcholinesterase activity in mice. J. Toxicol. Environ. Health, 26(4), 437-446. DOI: 10.1080/15287398909531267

23. Zhu, H., O'Brien, J.J., O'Callaghan, J.P., et al. (2010) Nerve Agent Exposure Elicits SiteSpecific Changes in Protein Phosphorylation in Mouse Brain. Brain Res., 1342, 11-23. DOI: 10.1016/j.brainres.2010.04.034

24. Michalovicz, L. T., Kelly, K. A., Sullivan, K., \& O'Callaghan, J. P. (2020). Acetylcholinesterase inhibitor exposures as an initiating factor in the development of Gulf War 
Illness, a chronic neuroimmune disorder in deployed veterans. Neuropharmacology, 171, 108073. DOI: $10.1016 /$ j.neuropharm.2020.108073

25. Miller, J.V., LeBouf, R.F., Kelly, K.A., et al. (2018) The Neuroinflammatory Phenotype in a Mouse Model of Gulf War Illness is Unrelated to Brain Regional Levels of Acetylcholine as Measured by Quantitative HILIC-UPLC-MS/MS. Toxicol. Sci., 165(2), 302-313. DOI: 10.1093/toxsci/kfy130

26. Hobson, B. A., Rowland, D. J., Supasai, S., et al. (2018). A magnetic resonance imaging study of early brain injury in a rat model of acute DFP intoxication. Neurotoxicology, 66, 170-178. DOI: 10.1016/j.neuro.2017.11.009

27. Sisó, S., Hobson, B.A., Harvey, D.J., et al. (2017) Editor's Highlight: Spatiotemporal Progression and Remission of Lesions in the Rat Brain Following Acute Intoxication With Diisopropylfluorophosphate. Toxicol. Sci., 157(2), 330-341. DOI: 10.1093/toxsci/kfx048

28. Vrana, J.A., Currie, H.N., Han, A.A., \& Boyd, J. (2014) Forecasting Cell Death Dose-Response from Early Signal Transduction Responses In Vitro. Toxicol. Sci., 140(2), 338-351. DOI: 10.1093/toxsci/kfu089

29. Farkhondeh, T., Mehrpour, O., Buhrmann, C., et al. (2020) Organophosphorus Compounds and MAPK Signaling Pathways. Int. J. Mol. Sci., 21(12), 4258. DOI: 10.3390/ijms21124258

30. Te, J.A., Spradling-Reeves, K.D., Dillman, J.F., \& Wallqvist, A. (2015) Neuroprotective mechanisms activated in non-seizing rats exposed to sarin. Brain Res., 1618, 136-148. DOI: 10.1016/j.brainres.2015.05.034

31. Cargnello, M. \& Roux, P.P. (2011) Activation and Function of the MAPKs and Their Substrates, the MAPK-Activated Protein Kinases. Microbiol. Mol. Biol. Rev., 75(1), 50-83. DOI: 10.1128/MMBR.00031-10

32. Li, L., Zhao, G.D., Shi, Z., et al. (2016) The Ras/Raf/MEK/ERK signaling pathway and its role in the occurrence and development of HCC. Oncol. Lett., 12(5), 3045-3050. DOI: 10.3892/ol.2016.5110

33. Busquets, O., Ettcheto, M., Cano, A., et al. (2020) Role of c-Jun N-Terminal Kinases (JNKs) in Epilepsy and Metabolic Cognitive Impairment. Int. J. Mol. Sci., 21(1), 255. DOI: 10.3390/ijms 21010255

34. Guma, M. \& Firestein, G.S. (2012) c-Jun N-Terminal Kinase in Inflammation and Rheumatic Diseases. Open Rheumatol. J., 6, 220-231. DOI: 10.2174/1874312901206010220

35. Caughlan, A., Newhouse, K., Namgung, U., \& Xia, Z. (2004) Chlorpyrifos induces apoptosis in rat cortical neurons that is regulated by a balance between p38 and ERK/JNK MAP kinases. Toxicol. Sci., 78(1), 125-134. DOI: 10.1093/toxsci/kfh038

36. Lee, J.E., Park, J.H., Jang, S.J., \& Koh, H.C. (2012) Reactive oxygen species regulated mitochondria-mediated apoptosis in PC12 cells exposed to chlorpyrifos. Toxicol. Appl. Pharm., 263(2), 148-162. DOI: 10.1016/j.taap.2012.06.005 
37. Lee, J.E., Park, J.H., Jang, S.J., \& Koh, H.C. (2014) Rosiglitazone inhibits chlorpyrifosinduced apoptosis via modulation of the oxidative stress and inflammatory response in SH-SY5Y cells. Toxicol. Appl. Pharm., 278(2), 159-171. DOI: 10.1016/j.taap.2014.04.021

38. Niijima, H., Nagao, M., Nakajima, M., et al. (2000) The effects of sarin-like and soman-like organophosphorus agents on MAPK and JNK in rat brains. Forensic Sci. Int., 112(2-3), 171-178. DOI: $10.1016 / \mathrm{s} 0379-0738(00) 00274-7$

39. Dhanasekaran, D.N. \& Reddy, E.P. (2008) JNK Signaling in Apoptosis. Oncogene, 27(48), 6245-6251. DOI: 10.1038/onc.2008.301

40. Pennington, K.L., Chan, T.Y, Torres, M.P., \& Andersen, J.L. (2018) The dynamic and stressadaptive signaling hub of 14-3-3: emerging mechanisms of regulation and context-dependent protein-protein interactions. Oncogene, 37(42), 5587-5604. DOI: 10.1038/s41388-018-0348-3

41. Sunayama, J., Tsuruta, F., Masuyama, N., \& Gotoh, Y. (2005) JNK antagonizes Akt-mediated survival signals by phosphorylating 14-3-3. J. Cell Biol., 170(2), 295-304. DOI: 10.1083/jcb.200409117

42. Mocsai, A., Ruland, J. \& Tybulewicz, V.L.J. (2016) The SYK tyrosine kinase: a crucial player in diverse biological functions. Nat. Rev. Immunol., 10(6), 387-402. DOI: 10.1038/nri2765

43. Geahlen, R.L. (2015) Getting SYK: spleen tyrosine kinase as a therapeutic target. Trends Pharmacol. Sci., 35(8), 414-422. DOI: 10.1016/j.tips.2014.05.007

44. Kanie, T., Abe, A., Matsuda, T., et al. (2004) TEL-Syk fusion constitutively activates PI3K/AKT, MAPK and JAK2-independent STAT5 signal pathways. Leukemia, 18, 548-555. DOI: 10.1038/sj.leu.2403266

45. Carnevale, J., Ross, L., Puissant, A., et al. (2013) SYK regulates mTOR signaling in AML. Leukemia, 27(11), 2118-2128. DOI: 10.1038/leu.2013.89

46. Parsons, S.J. \& Parsons, J.T. (2004) Src family kinases, key regulators of signal transduction. Oncogene, 23, 7906-7909. DOI: 10.1038/sj.onc.1208160

47. Liu, D., Zhang, X., Hu, B.L., \& Ander, B.P. (2016) Src Family Kinases in Brain Edema after Acute Brain Injury. Acta. Neurochir. Suppl., 121, 185-190. DOI: 10.1007/978-3-319-18497-5_33

48. Liu, D.Z., Sharp, F.R., Van, K.C., et al. (2014) Inhibition of Src Family Kinases Protects Hippocampal Neurons and Improves Cognitive Function after Traumatic Brain Injury. J. Neurotrauma, 31(14), 1268-1276. DOI: 10.1089/neu.2013.3250

49. Park, Y., Luo, T., Zhang, F., et al. (2013) Downregulation of Src-kinase and glutamate-receptor phosphorylation after traumatic brain injury. J. Cereb. Blood Flow Metab., 33(10), 1642-1649. DOI: $10.1038 / \mathrm{jcbfm} .2013 .121$

50. Salter, M.W. \& Kalia, L.V. (2004) Src kinases: a hub for NMDA receptor regulation. Nat. Rev. Neurosci., 5(4), 317-328. DOI: 10.1038/nrn1368 
51. Zhou, Q. \& Sheng, M. (2013) NMDA receptors in nervous system diseases. Neuropharmacology, 74, 69-75. DOI: 10.1038/nrn1368

52. Lowell, C.A. (2011) Src-family and Syk Kinases in Activating and Inhibitory Pathways in Innate Immune Cells: Signaling Cross Talk. Cold Spring Harb. Perspect. Biol., 3(3), a002352. DOI: $10.1101 /$ cshperspect.a002352 


\section{Chapter 4: Corticosterone and chlorpyrifos oxon exposure elicits spatiotemporal MAPK phosphoprotein signaling in a mouse brain}

Parts of this chapter have been published previously: Julia A. Penatzer, Nicole Prince, Julie V. Miller, Mackenzie Newman, Cayla Lynch, Gerald R. Hobbs, Jonathan W. Boyd (2021) Corticosterone and chlorpyrifos oxon exposure elicits spatiotemporal MAPK phosphoprotein signaling in a mouse brain. Food and Chemical Toxicology, 155, 112421. https://doi.org/10.1016/j.fct.2021.112421

Chlorpyrifos (CPF) is one of the most widely-used organophosphate pesticides globally for agricultural purposes. Certain occupations (e.g., field workers, farmers) are at an increased risk for high-dose exposure to CPF, which can lead to seizures and irreversible brain injury. Workers within these occupations also experience increased circulating cortisol levels, which is related to physiological stress. To better represent this exposure scenario, a mouse model utilized exogenous administration of corticosterone (CORT; high physiologic stress mimic) in combination with chlorpyrifos oxon (CPO; oxon metabolite of $\mathrm{CPF}$ ); this combination increases neuroinflammation and acetylcholine post-exposure. In the present study adult male C57BL/6J mice were given CORT $(200 \mu \mathrm{g} / \mathrm{mL})$ in drinking water for seven days followed by a single intraperitoneal injection of CPO $(8.0 \mathrm{mg} / \mathrm{kg})$ on day eight, and euthanized $0.5,2$, and $24 \mathrm{~h}$ post-injection. Ten posttranslationally modified proteins were measured in the cortex and striatum to evaluate brain region-specific effects. The spatiotemporal response to $\mathrm{CORT}+\mathrm{CPO}$ sequentially activated phosphoproteins (p-ERK1/2, p-MEK1/2, p-JNK) involved in mitogen-activated protein kinase (MAPK) signaling. Observed p-ZAP70 responses further integrated MAPK signaling and provided a spatiotemporal connection between protein phosphorylation and neuroinflammation. This study provides insight into the spatiotemporal cellular signaling cascade following CORT+CPO exposure that better represent these vulnerable populations. 


\subsection{Introduction}

Chlorpyrifos $(\mathrm{CPF})$ is one of the most widely used organophosphate insecticides, and approximately eight million pounds are applied annually to both agricultural (e.g., corn, soybeans, cotton, grain) and non-agricultural areas (e.g., golf courses, green houses) in the United States alone [1-3]. CPF is effective in eliminating pests, but poses serious human health risks [4-5]. Mild exposures can result in headaches, blurry vision, salivation, and muscle weakness, while highlevel exposures can lead to difficulty breathing, convulsions, irregular heartbeat, coma, and even death [6]. Prolonged exposure to CPF has been linked to chronic diseases (e.g., cancer, neurological disorders) and persistent neuroinflammation [7-9]. Despite the toxicity associated with $\mathrm{CPF}$, its use in agricultural applications has increased worldwide because of its low cost and easy access [10], which poses a concern for the wellbeing of agricultural workers. Approximately $25 \%$ of the world's population works in an agriculturally related field, which is predominated by individuals in lower socioeconomic classes [11-12]. These individuals are vulnerable to malnutrition and high levels of stress, both of which increase circulating cortisol [12-18]. However, few studies have addressed the mixed exposure profile of high physiological stress with CPF to better model vulnerable populations that are at an increased risk for exposure in combination with increased and prolonged cortisol levels.

In order to better understand the combinatorial exposure of CPF and stress, Locker et al. (2017) developed a mouse model involving pre-treatment with the rodent surrogate for cortisol, corticosterone (CORT), to mimic a high stress environment, followed by exposure to chlorpyrifos oxon (CPO), the active metabolite of CPF that inhibits acetylcholinesterase (AChE) [7]. This combination of CORT+CPO enhanced neuroinflammation; levels of cytokines and chemokines were higher within the first six hours in the cortex and striatum when compared to controls (CPO 
or CORT alone) [7, 19]. Miller et al. (2018), used the same model to display spatial differences in acetylcholine concentrations and found that CORT+CPO led to significant increases in the striatum, but no significant differences in the cortex [19]. However, several studies have suggested the toxicity associated with CPF is independent of AChE inhibition, but leads to multiple cellular signaling cascades that promotes apoptosis [20-23].

Previous research has shown increases in brain STAT3 phosphorylation relative to controls following CORT+CPO exposure [7]. STAT3 is crucial to the Janus kinases/signal transducers and activators of transcription (JAK/STAT) pathway, which is activated by numerous cytokines, but also impacts several downstream signaling cascades (e.g., mitogen-activated protein kinase; MAPKs) known to integrate cellular stress responses [24-25]. To build upon this previous work, this study investigated key post-translational modifications involved in these pathways to elucidate the cellular response mechanisms relevant to CPF exposure with stress. By specifically monitoring early phosphorylation events in the context of previous cytokine and acetylcholine data in the brain, we aim to disentangle the toxic mechanisms associated with neuroinflammation caused by hormones (i.e., CORT in this model) from those associated with organophosphate pesticide exposures (i.e., $\mathrm{CPO}$ in this model). In this study, adult male $\mathrm{C} 57 \mathrm{BL} / 6 \mathrm{~J}$ mice were exposed to CORT $(200 \mu \mathrm{g} / \mathrm{mL})$ in drinking water for seven days, then given a single intraperitoneal (i.p.) injection of $\mathrm{CPO}(8.0 \mathrm{mg} / \mathrm{kg})$ on day eight. Mice were euthanized at $0.5,2$, and $24 \mathrm{~h}$ post-exposure via focused microwave irradiation to preserve the steady-state levels of protein phosphorylation [26]. To fully understand the effects of CORT+CPO on specific regions of the brain, 10 posttranslationally modified protein targets involved in MAPK signaling were measured using a multiplex bead-based enzyme-linked immunosorbent assay (ELISA) for the cortex and striatum. 
This approach provided insight to the effects observed post-high stress and pesticide exposure based on the elucidation of relevant spatiotemporal phosphorylation responses.

\subsection{Materials and methods}

\subsubsection{Animals}

All procedures were performed under protocols approved by the Institutional Animal Care and Use Committee of the Centers for Disease Control and Prevention, National Institute for Occupational Safety and Health and the Animal Care and Use Review Office from the Department of Defense. The animal facility was certified by the American Association for Accreditation of Laboratory Animal Care. Adult male C57BL/6J mice (approximately 8-12 weeks of age) were purchased from Jackson Laboratory (Bar Harbor, ME, USA) and were individually housed in a temperature $\left(21 \pm 1^{\circ} \mathrm{C}\right)$ and humidity-controlled $(50 \pm 10 \%)$ colony room that was maintained under filtered positive-pressure ventilation on a $12 \mathrm{~h}$ light/12 h dark cycle. Mice were given ad libitum access to food and water.

\subsubsection{Dosing}

Mice ( $\mathrm{n}=\mathrm{at}$ least five/group) received CORT (Steraloids Inc., Newport, RI, USA) in the drinking water (200 $\mu \mathrm{g} / \mathrm{mL}$ in 1.2\% ethanol, Sigma-Aldrich Co.) for seven days (Figure 4.1) based on previous research shown to achieve high circulating levels of the hormone [27-28]. Control groups received $1.2 \%$ ethanol in the drinking water. On day eight, mice received a single i.p. injection of peanut oil or CPO $(8.0 \mathrm{mg} / \mathrm{kg}$, Chem Service, Inc., West Chester, PA, USA; Figure 4.1), which produced symptoms of cholinergic crisis (e.g., SLUD [salivation, lacrimation, urination, and defecation] and seizures) while displaying mortality below LD25, in accordance with Locker et al. (2017) [7]. CPO (the oxon metabolite of CPF) was used in place of CPF to avoid 
animal variability related to the bioactivation of CPF, in line with previous research [29-30]. Mice were then returned to their home cage prior to sacrifice.

Preliminary power analyses $(\alpha=0.05)$ revealed that an $\mathrm{N}=4$ was sufficient for this study to determine phosphoprotein significant differences between exposure groups. Following exposure, peanut oil $(\mathrm{N}=5)$, CORT $(\mathrm{N}=5)$, and $\mathrm{CPO}(\mathrm{N}=7)$ exposed mice survived all of the time points, but only 5 of 7 mice from the CORT+CPO exposure survived at the $2 \mathrm{~h}$ and $24 \mathrm{~h}$ time points $(71 \%$ survival rate). Mice that did not survive to study termination were excluded from analysis.

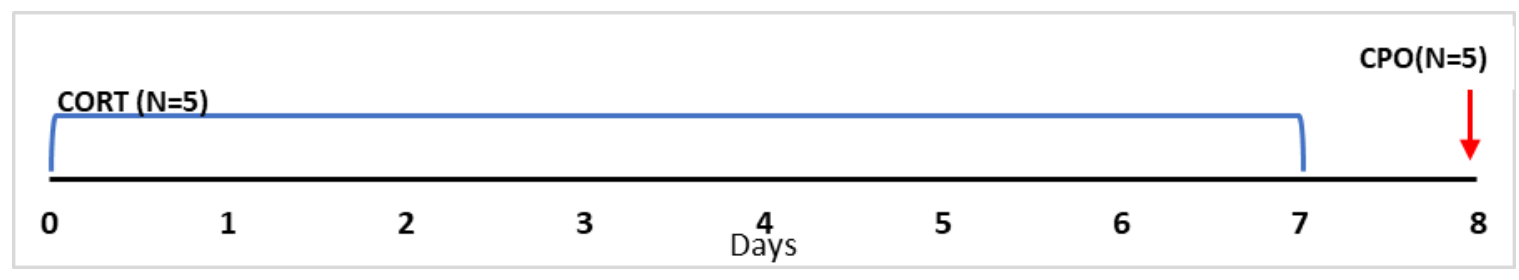

Figure 4.1. Dosing paradigm for corticosterone and chlorpyrifos oxon exposure. Timeline for administration of chlorpyrifos oxon (CPO $8.0 \mathrm{mg} / \mathrm{kg})$ after corticosterone (CORT) priming $(200 \mu \mathrm{g} / \mathrm{mL}$ in $1.2 \%$ ethanol) in drinking water.

\subsubsection{Brain dissection}

Mice were sacrificed at three time points $(0.5,2$, and $24 \mathrm{~h}$ post-exposure $)$ by focused microwave irradiation to preserve the steady-state levels of protein phosphorylation and provide a snapshot of the cell signaling alterations in response to these exposures [26]. Whole brains were removed from the skull and dissected free-hand with fine-curved forceps on a thermoelectric cold plate (Model TCP-2; Aldrich Chemical Co., Milwaukee WI) into the cortex and striatum. The cortex and striatum were chosen based on previous cytokine and acetylcholine data determined from this model [7, 19]. The striatum has a higher density of acetylcholine and AChE than the cortex, which may have discrete brain-region differences in the resulting phosphoprotein signaling [31-33]. Samples were immediately rinsed with ice cold phosphate buffered saline, snap frozen, and stored at $-80^{\circ} \mathrm{C}$ until analysis 


\subsubsection{Protein quantification}

Methods from Hulse et al. (2004) were adapted for protein extraction [34]. Specifically, one side of each brain region (e.g., left cortex) was placed into a tube with $500 \mu \mathrm{L}$ of Bio-Rad cell lysis buffer containing a protease inhibitor cocktail (Bio-Rad Factor 1 and 2; Bio-Rad, Hercules, CA) and $2 \mathrm{mM}$ phenylmethylsulfonyl fluoride (Sigma, St. Louis, MO). Samples were homogenized on ice with three rapid pulses for three seconds at 35\% amplitude (Fisher Scientific, Pittsburgh, PA). Samples were centrifuged at $4,500 \mathrm{x}$ g for $4 \mathrm{~min}$ at $4^{\circ} \mathrm{C}$, and then the supernatant was collected into a sterile tube and stored at $-80^{\circ} \mathrm{C}$. Total protein concentration was determined using the Pierce protein assay (Fisher Scientific, Pittsburgh, PA), according to the manufacturer's instructions. Absorbance values were determined at $562 \mathrm{~nm}$ using an Infinite M1000 plate reader (Tecan, Raleigh, NC).

\subsubsection{Phosphoprotein analysis in discrete brain regions}

For phosphoprotein analysis, sample homogenates were diluted to a total protein concentration of $700 \mu \mathrm{g} / \mathrm{mL}$ with sample diluent (Bio-Rad, Hercules, CA). Samples were assayed using a custom Bio-Plex Pro multiplexed magnetic bead-based immunoassay reagent kit for the following phosphorylated targets at the indicated amino acid residues: p-BAD (S136), p-p70 S6 Kinase (T389; p-p70S6K), p-S6 Ribosomal P (S235/S236; p-RPS6), p-ZAP70 (Y319), p-CREB (S133), p-ERK1/2 (T202/Y204, T185/Y187), p-GSK3 $\alpha / \beta$ (S21/S9), p-IкB $\alpha$ (S32/S36), p-JNK (T183/Y185), and p-MEK1/2 (S217/S221). All samples were analyzed with a Bio-Plex 200 suspension array system and Pro II Wash Station (Bio-Rad, Hercules, CA), according to the manufacturer's instructions. Biological replicates were at least in quadruplicate and all measurements were performed in duplicate (technical replicates). Bio-Rad lysate positive (EGFtreated HEK-293, UV-treated HEK-293, EGF-treated SK-BR-3, TNF- $\alpha$-treated HeLa, NGF- $\beta$ - 
treated PC12, H2O2-treated Jurkat, and PDGF-treated NIH3T3) and negative (phosphatase-treated HeLa) controls were used to ensure appropriate bead counts.

\subsubsection{Statistical analysis}

All data analyses were performed using GraphPad Prism (V5; San Diego, CA, USA). Averaged raw values were blank-subtracted and any targets which showed a negative phosphorylation response (target < blank) were removed before analysis. Relative phosphorylation was determined for each target by normalizing to the peanut oil controls. A Grubbs' test was performed to remove any significant outliers $(\alpha=0.05)$. As previously mentioned, any nonsurviving animals were also excluded from analysis. The normalized phosphorylation \pm the standard error of the mean (SEM) for each exposure and time point was used to identify significant targets. Two-way analysis of variance (ANOVA) with Bonferroni post-tests were performed to determine significance $(\mathrm{p}<0.05)$.

\subsection{Results}

\subsubsection{Relative phosphorylation responses in the cortex}

In the cortex, CORT $+\mathrm{CPO}$ exposure elicited a significant response $(\mathrm{p}<0.05)$ for all measured phosphoproteins compared to the controls for at least one time point (Figure 4.2). CORT+CPO caused a significant $(\mathrm{p}<0.05)$ increase in phosphorylation of RPS6 at $0.5 \mathrm{~h}$ postexposure relative to CORT alone. At $2 \mathrm{~h}, \mathrm{CORT}+\mathrm{CPO}$ resulted in significantly $(\mathrm{p}<0.05)$ increased phosphorylation of p-p70S6K compared to CORT alone. Phosphorylated CREB, ERK1/2, GSK $3 \alpha / \beta$, I $\kappa \mathrm{B} \alpha$, and MEK1/2 were also significantly $(\mathrm{p}<0.05)$ increased for CORT $+\mathrm{CPO}$ relative to $\mathrm{CPO}$ alone (Figure 4.2). Conversely, at $24 \mathrm{~h}$, p-ERK1/2 was significantly $(\mathrm{p}<0.05)$ decreased for CORT+CPO compared to peanut oil, while $\mathrm{p}-\mathrm{BAD}$ and $\mathrm{p}-\mathrm{MEK} 1 / 2$ were significantly $(\mathrm{p}<0.05)$ decreased in response to CORT+CPO relative to all of the controls: peanut oil, CORT, and CPO 
(Figure 4.2). At $24 \mathrm{~h}, \mathrm{p}-\mathrm{JNK}$ and p-ZAP70 were significantly ( $\mathrm{p}<0.05)$ increased post-CORT+CPO exposure (Figure 4.2).

Temporally, several phosphoproteins were significantly $(\mathrm{p}<0.05)$ increased in response to $\mathrm{CORT}+\mathrm{CPO}$ or CORT alone at $2 \mathrm{~h}$ or $24 \mathrm{~h}$ post-exposure (Figure 4.2). CORT $+\mathrm{CPO}$ resulted in significant $(\mathrm{p}<0.05)$ increases at $2 \mathrm{~h}$ relative to $24 \mathrm{~h}$ for $\mathrm{p}-\mathrm{p} 70 \mathrm{~S} 6 \mathrm{~K}$ and $\mathrm{p}-\mathrm{GSK} 3 \alpha / \beta$. Similarly, $\mathrm{p}$ CREB, p-ERK1/2, and p-MEK1/2 were significantly $(\mathrm{p}<0.05)$ increased for CORT+CPO exposure at $2 \mathrm{~h}$ relative to 0.5 and $24 \mathrm{~h}$ (Figure 4.2). Phosphorylated BAD and IkB $\alpha$ were significantly $(\mathrm{p}<0.05)$ decreased at $24 \mathrm{~h}$ compared to the early time points following CORT+CPO exposure, whereas JNK was significantly $(\mathrm{p}<0.05)$ phosphorylated (Figure 4.2). CORT alone also resulted in a significant $(\mathrm{p}<0.05)$ increase at the $2 \mathrm{~h}$ time point for $\mathrm{p}$-ERK1/2. 


\section{CORTEX}
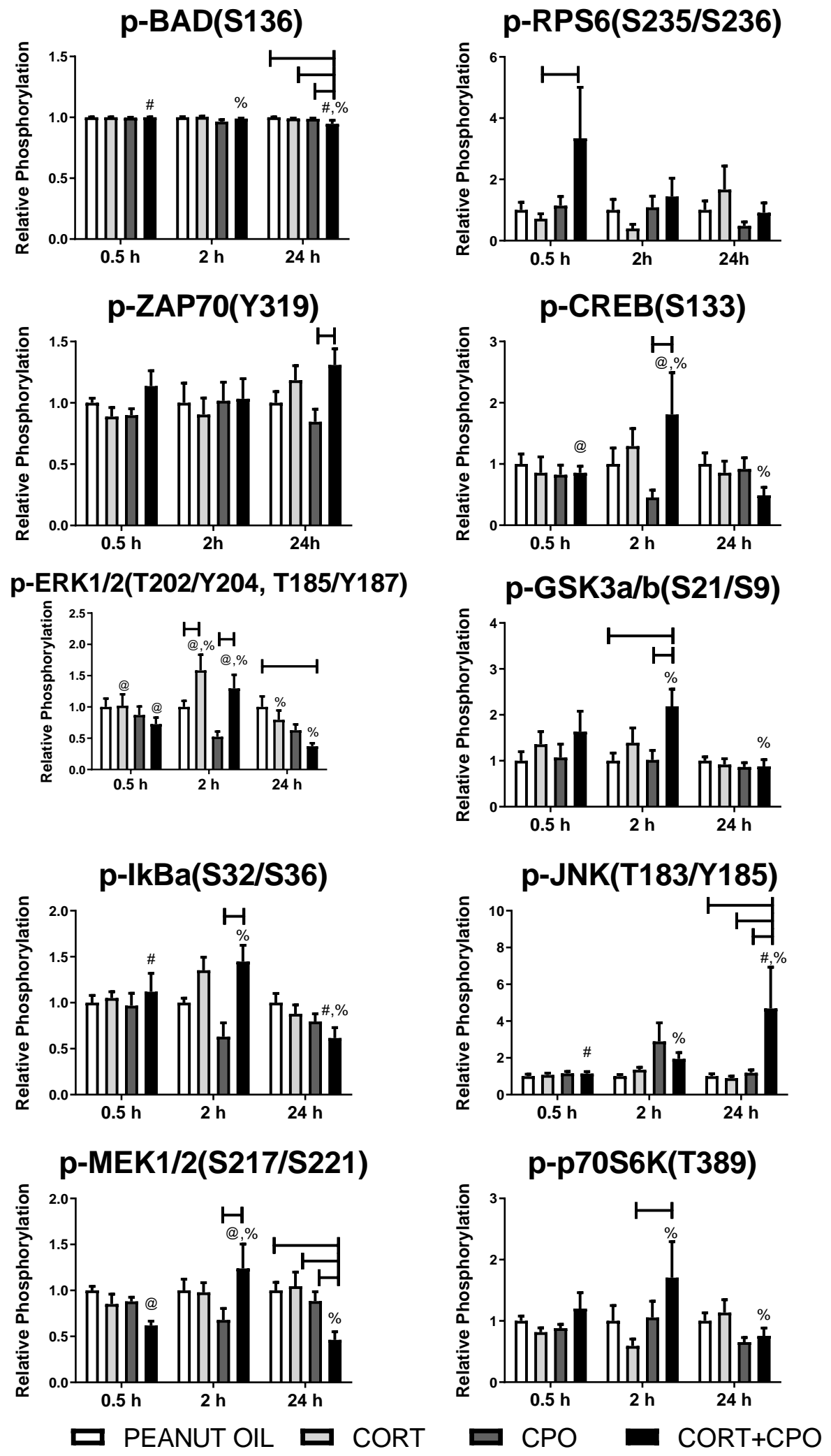
Figure 4.2. Significant phosphoprotein responses in the cortex via multiplex ELISA post-exposure to corticosterone and chlorpyrifos oxon. Mice were exposed to control or $200 \mu \mathrm{g} / \mathrm{mL}$ CORT in drinking water for seven days. On day eight, mice were given a single injection of CPO (8 mg/kg, i.p.) or peanut oil (Figure 4.1) and sacrificed via focused microwave irradiation at $0.5,2$, or $24 \mathrm{~h}$ post-exposure. Data are represented as mean $\pm \mathrm{SEM}$ with at least $\mathrm{N}=4$ for all phosphoproteins. Two-way ANOVA with Bonferroni post-test was used for determining significance, where @ indicates a significant difference $(\mathrm{p}<0.05)$ between $0.5 \mathrm{~h}$ vs. $2 \mathrm{~h}$, \# indicates a significant difference $(\mathrm{p}<0.05)$ between $0.5 \mathrm{~h}$ vs. $24 \mathrm{~h}$, and $\%$ indicates a significant difference $(\mathrm{p}<0.05)$ between $2 \mathrm{~h}$ vs. $24 \mathrm{~h}$ for a particular exposure. Horizontal bars represent significance $(\mathrm{p}<0.05)$ between exposures within a specific time.

\subsubsection{Relative phosphorylation responses in the striatum}

The striatum only had two phosphoproteins that were significantly $(\mathrm{p}<0.05)$ affected by an exposure (Figure 4.3). Phosphorylated BAD was significantly ( $\mathrm{p}<0.05)$ decreased for CORT+CPO exposure at $0.5 \mathrm{~h}$ relative to peanut oil and $\mathrm{CPO}$ (Figure 4.3). Similar to the cortex, CORT+CPO resulted in a significant $(\mathrm{p}<0.05)$ increase in phosphorylation of $\mathrm{p}-\mathrm{JNK}$ at $24 \mathrm{~h}$ (Figure 4.3 ) relative to peanut oil and CORT alone.

Temporally, CORT $+\mathrm{CPO}$ exposure significantly $(\mathrm{p}<0.05)$ increased MEK1/2 $(\mathrm{p}<0.05)$ phosphorylation at $0.5 \mathrm{~h}$ relative to $24 \mathrm{~h}$. At $2 \mathrm{~h}, \mathrm{p}-\mathrm{BAD}$ was significantly increased $(\mathrm{p}<0.05)$ for CORT+CPO compared to 0.5 and 24 h post-exposure (Figure 4.3). Phosphorylated ZAP70 and JNK were both significantly $(\mathrm{p}<0.05)$ increased at $24 \mathrm{~h}$ post-exposure for CORT $+\mathrm{CPO}$ (Figure 4.3). CORT alone significantly ( $\mathrm{p}<0.05)$ increased $\mathrm{p}$-p70S6K at the $24 \mathrm{~h}$ time point (Figure 4.3 ). $\mathrm{CPO}$ alone also led to significant $(\mathrm{p}<0.05)$ changes in phosphoprotein responses in the striatum: p-MEK1/2 and p-BAD were significantly $(\mathrm{p}<0.05)$ decreased at $24 \mathrm{~h}$ relative to $0.5 \mathrm{~h}$ and $2 \mathrm{~h}$, respectively, while p-p70S6K was significantly $(\mathrm{p}<0.05)$ increased at $24 \mathrm{~h}$ post-exposure compared to $2 \mathrm{~h}$ (Figure 4.3). 


\section{STRIATUM}
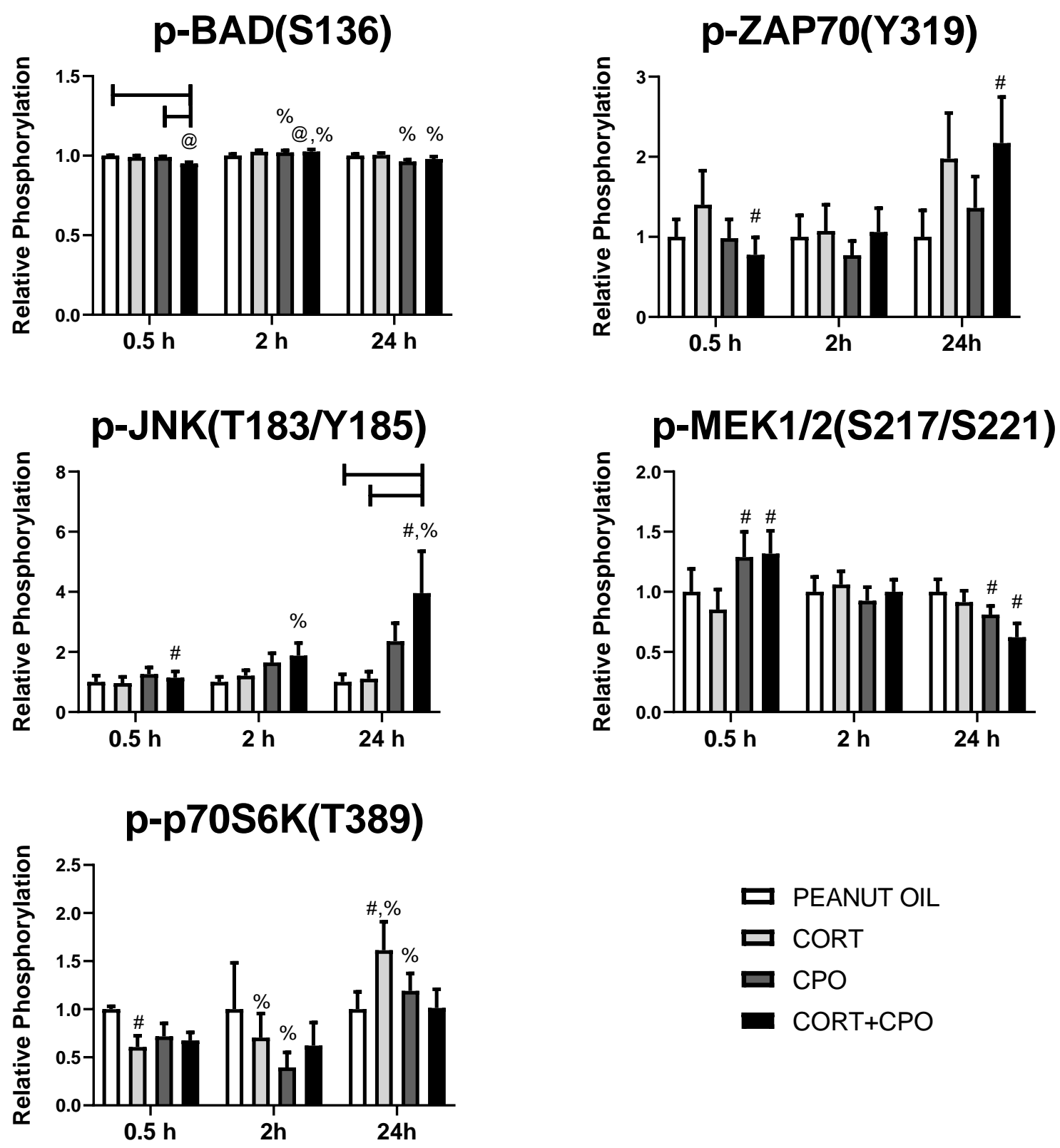

Figure 4.3. Significant phosphoprotein responses in the striatum via multiplex ELISA post-exposure to corticosterone and chlorpyrifos oxon. Mice were treated with the same dosing regimen and sacrifice as stated in Figure 4.1. Twoway ANOVA with Bonferroni post-test were used for determining significance, where the horizontal bars represent significance $(\mathrm{p}<0.05)$ between exposures within a specific time. Temporally, @ indicates a significant difference 
$(\mathrm{p}<0.05)$ between $0.5 \mathrm{~h}$ vs. $2 \mathrm{~h}$, \# indicates a significant difference $(\mathrm{p}<0.05)$ between $0.5 \mathrm{~h}$ vs. $24 \mathrm{~h}$, and \% indicates a significant difference $(\mathrm{p}<0.05)$ between $2 \mathrm{~h}$ vs. $24 \mathrm{~h}$ for a particular exposure. Data are represented as mean \pm SEM.

\subsection{Discussion}

CPF remains in the top 15 of the most-widely used pesticides worldwide, but presents serious health risks, especially to those employed in agriculture [35-37]. As such, numerous studies point to the detrimental effects of $\mathrm{CPF}$ alone. To better represent the vulnerable populations in agriculture who are most likely to be exposed to high concentrations of CPF and carry the burden of chronically increased cortisol, this combinatorial exposure scenario should be further explored [12-18]. Previous rodent studies using CORT+CPO exposure found increases in neuroinflammation post-exposure $[7,19]$. Thus, an investigation into early intracellular signaling responses via protein phosphorylation in discrete brain regions (i.e., cortex and striatum) was performed to discern the pathways that may reveal biomolecular drivers of neuroinflammation and toxicity associated with this exposure scenario involving a susceptible population.

Early cellular post-translational phosphorylation events regulate adaptive stress and inflammatory pathways that are dependent on both spatial location and time to initiate the appropriate cell signaling cascades [38-40]. By analyzing discrete tissue regions, we were able to discriminate local xenobiotic toxicity that may otherwise go undetected in pooled brain samples [31]. Moreover, the temporal response from the phosphoproteins tested in this study provided valuable insight into the changes in MAPK signaling cascades at earlier time points $(0.5$ and $2 \mathrm{~h}$ post-exposure) versus the delayed phosphoprotein response ( $24 \mathrm{~h})$. In the cortex, at the early time points post-exposure to $\mathrm{CORT}+\mathrm{CPO}$, there were significant $(\mathrm{p}<0.05)$ increases in the phosphorylation of proteins in the MEK/ERK cascade: p-RPS6 was significantly increased at 0.5 $\mathrm{h}$, while p-MEK1/2, p-ERK1/2, p-p70S6K, and p-CREB are all significantly increased at $2 \mathrm{~h}$ for $\mathrm{CORT}+\mathrm{CPO}$ relative to the controls (Figure 4.2). This cascade couples' signals from cell-surface 
cytokine (e.g., interleukin (IL)-3, granulocyte/macrophage colony stimulating factor [GM-CSF]) receptors to key downstream pathways involved in cell proliferation and differentiation [41-42]. These cytokines trigger the phosphorylation of MEK1/2 and ERK1/2, which, in turn, have been shown to phosphorylate p70S6K, RPS6, and CREB (Figure 4.4) [43-45]. Previous research has implicated the MEK/ERK cascade in vitro following CPF or CPO exposure alone, in agreement with the present study [20-21, 46-48].

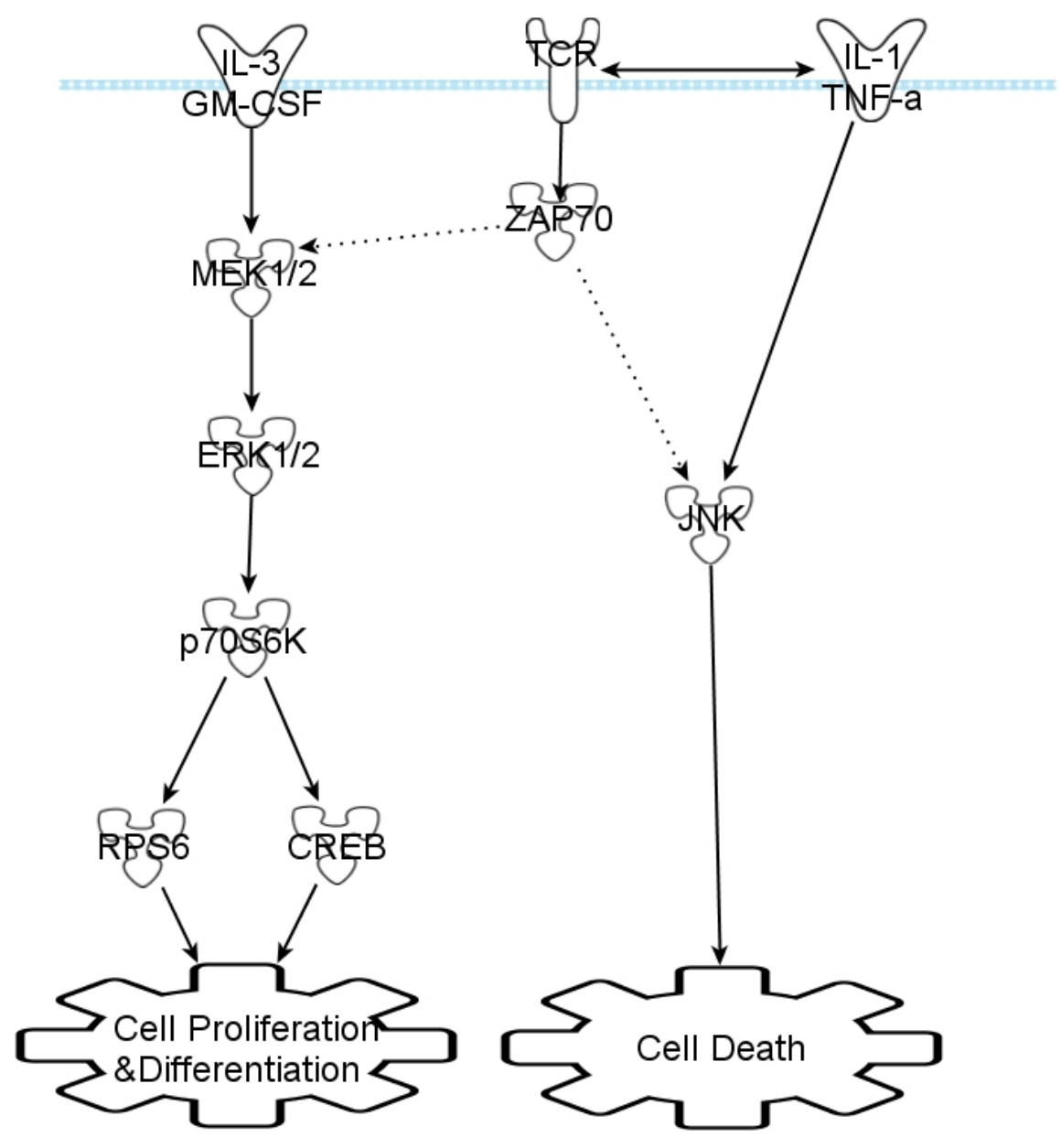

Figure 4.4. MAPK signaling pathway following corticosterone with chlorpyrifos oxon exposure. Activation of the Tcell receptor (TCR) increases activity of ZAP70, which promotes activation of the MAPK pathway (i.e., JNK, MEK/ERK). The MEK/ERK pathway can also be stimulated by GM-CSF and IL-3. Activation of this pathway results in phosphorylation of MEK1/2 and ERK1/2, which, in turn, phosphorylate p70S6K, RPS6, and CREB promoting cell 
proliferation and differentiation. In contrast, JNK phosphorylation eventually results in cell death. JNKs can also be activated by IL-1 and TNF- $\alpha$. The solid lines represent a direct effect on phosphorylation between proteins, whereas the dotted line indicates an indirect connection

By continuing to monitor the post-exposure time course to $24 \mathrm{~h}$, we can further evaluate how previously-reported cytokine responses (e.g., tumor necrosis factor- $\alpha$ (TNF- $\alpha$ ), interleukin (IL)-1) from $6 \mathrm{~h}$ post-exposure to CORT+CPO alter the intracellular signaling response [7, 19]. Our results demonstrated a shift in MAPK signaling in the cortex at $24 \mathrm{~h}$ post-exposure to $\mathrm{CORT}+\mathrm{CPO}$; $\mathrm{p}-\mathrm{JNK}$ is activated in response to $\mathrm{CORT}+\mathrm{CPO}$ at the later time point concurrent with MEK/ERK phosphorylation decreases (Figure 4.2). Phosphorylated JNK is also significantly increased in the striatum to CORT+CPO exposure at $24 \mathrm{~h}$ (Figure 4.3). The JNK pathway is activated by environmental stressors and inflammatory cytokines (e.g., TNF- $\alpha$, IL-1) and has been shown to have a central role in apoptosis (Figure 4.4) [48-51]. Therefore, these increases in TNF$\alpha$ and IL-1 in the cortex and striatum may be leading to the activation of p-JNK observed in the present study.

In a study by Lazar et al. (2016), exposure to sarin, which is an irreversible AChE inhibitor, resulted in a temporary increase in ERK1/2, but saw no differences with JNK response in a rat's cortex; however, at $24 \mathrm{~h}$, ERK1/2 was decreased and JNK was increased, similar to the present study (Figure 4.2) [52]. Moreover, JNKs have been implicated in several studies post-exposure to CPF or CPO to initiate apoptosis, which may lead to the development of neurodegenerative disorders [20-21, 53-54]. Interestingly, inhibition or decreases in p-ERK1/2 post-exposure to CPF has also been linked to delayed neurotoxicity [55]. These MAPK signaling related significant phosphoprotein findings have also been demonstrated with CPO or CPF alone, but, in the present study, there were no significant differences with CPO alone (Figures 4.2, 4.3). However, $\mathrm{CORT}+\mathrm{CPO}$ initiates these pathways, indicating that $\mathrm{CPO}$ exposure with CORT priming is more 
sensitive to neuroinflammation and results in activation of this apoptotic pathway. Therefore, in the work presented here, the initial response to CORT+CPO may inhibit apoptosis (e.g., increased p-ERK1/2, p-MEK1/2), but by $24 \mathrm{~h}$ this phosphoprotein signaling is indicative of cell death (e.g., decreased p-ERK1/2 and increased p-JNK).

To further evaluate the relationship between neuroinflammation, immune responses, MAPK signaling, and CORT+CPO exposure p-ZAP70 was measured. ZAP70 plays a crucial role in regulating downstream signaling events involved in MAPK pathways (Figure 4.4), and is linked to the release of inflammatory mediators (e.g., TNF- $\alpha$, IL-6), protein tyrosine phosphorylation, and activation of $\mathrm{T}$ cells as phosphorylation is required in order to initiate $\mathrm{T}$ cell signaling [56-61]. In the present study, p-ZAP70 was found to be significantly $(\mathrm{p}<0.05)$ increased at $24 \mathrm{~h}$ postexposure to $\mathrm{CORT}+\mathrm{CPO}$ in the cortex (Figure 4.2) and the striatum (Figure 4.3). Therefore, ZAP70 provides an interesting link to previously observed inflammation through T cell signaling and the activation of JNK.

\subsection{Conclusion}

In summary, a mouse model was used to investigate the early cellular signaling cascade post-exposure to CORT and $\mathrm{CPO}$ to better represent the exposure scenario associated with a vulnerable population with chronically elevated cortisol who are exposed to CPF. Within this study it was determined that $\mathrm{CORT}+\mathrm{CPO}$ initiates distinct spatiotemporal phosphoprotein signaling responses. The cortex appears to be sequentially activating phosphoproteins involved in MAPK signaling: the MEK/ERK cascade is first phosphorylated in response to this combined exposure, but at $24 \mathrm{~h}$ these phosphoproteins are decreased. Phosphorylated JNK is increased at $24 \mathrm{~h}$ for both brain region regions, suggesting the initiation of cell death via apoptosis. ZAP-70, which functions upstream of MAPK signaling, provides an interesting biomarker in regard to the connection 
between $\mathrm{CORT}+\mathrm{CPO}$ exposure and neuroinflammation via $\mathrm{T}$-cell stimulation, which may be related to the increased toxicity observed in response to the combination of both exogenous (i.e., CPO) and endogenous (i.e., CORT) stressors. Future studies will further probe these proteins and their post-translational modifications at later time points and different doses of CPO to better understand the long-term effects associated with this exposure scenario involving a vulnerable population.

\subsection{Acknowledgements}

The authors gratefully acknowledge James P. O'Callaghan, Kimberly A. Kelly, Lindsay T. Michalovicz, Brenda K. Billig, Christopher M. Felton, Ali Yilmaz, and Fang Ma for assistance with dosing the mice and dissecting the brains. The authors would also like to thank Suzanne Danley for her editorial suggestions. This work was supported by Congressionally Directed Medical Research Programs: Gulf War Illness Research Program Grants (GW170081). Opinions, interpretations, conclusions, and recommendations are those of the author and are not necessarily endorsed by the Department of Defense. 


\subsection{References}

1. Robb, E.L. \& Baker, M.B. (2020) Organophosphate Toxicity. In: StatPearls [Internet]. Treasure Island (FL): StatPearls Publishing. Available from: https://www.ncbi.nlm.nih.gov/books/NBK470430/

2. Environmental Protection Agency (2020). Chlorpyrifos. Available from: https://www.epa.gov/ingredients-used-pesticide-products/chlorpyrifos

3. Sasikala, C., Jiwal, S., Rout, P., \& Ramya, M. (2012) Biodegradation of chlorpyrifos by bacterial consortium isolated from agriculture soil. World J. Microbiol. Biotechnol., 28, 1301-1308. DOI: $10.1007 / \mathrm{s} 11274-011-0879-\mathrm{z}$

4. Rahman, H.U., Asghar, W., Nazir, W., et al. (2021) A comprehensive review on chlorpyrifos toxicity with special reference to endocrine disruption: Evidence of mechanisms, exposures and mitigation strategies. Science of the Total Environment, 755(Part 2), 142649. DOI: 10.1016/j.scitotenv.2020.142649

5. Smegal, D.C. (2000) Human Health Risk Assessment: Chlorpyrifos. US Environmental Protection Agency. Available from: https://archive.epa.gov/scipoly/sap/meetings/web/pdf/hed_ra.pdf

6. Agency for Toxic Substances and Disease Registry (2011) ToxFAQs for Chlorpyrifos. Available from: https://wwwn.cdc.gov/TSP/ToxFAQs/ToxFAQsDetails.aspx?faqid=494\&toxid=88\#: :text=Exp osure $\% 20$ to $\% 20$ high $\% 20$ levels $\% 20$ may,or\%20causes $\% 20$ human $\% 20$ birth $\% 20$ defects.

7. Locker, A.R., Michalovicz, L.T., Kelly, K.A., et al. (2017) Corticosterone primes the neuroinflammatory response to Gulf War Illness-relevant organophosphates independently of acetylcholinesterase inhibition. J. Neurochem., 142(3), 444-455. DOI: 10.1111/jnc.14071

8. Seiber, J.N. \& Kleinschmidt, L.A. (2011) Contributions of pesticide residue chemistry to improving food and environmental safety: past and present accomplishments and future challenges. J. Agric. Food Chem., 59(14), 7536-7543. DOI: 10.1021/jf103902t

9. Talan, A., Mishra, A., Eremin, S.A., et al. (2018) Ultrasensitive electrochemical immunosensing platform based on gold nanoparticles triggering chlorpyrifos detection in fruits and vegetables. Biosensors and Bioelectronics, 105, 14-21. DOI: 10.1016/j.bios.2018.01.013

10. John, E.M. \& Shaike, J.M. (2015) Chlorpyrifos: pollution and remediation. Environ. Chem. Lett., 13, 269-291. DOI: 10.1007/s10311-015-0513-7

11. The World Bank (2021) Employment in agriculture (\% of total employment) (modeled ILO estimate).

Available

from:

https://data.worldbank.org/indicator/SL.AGR.EMPL.ZS?end=2020\&start=2018

12. Gillespie, S. \& van den Bold, M. (2017) Agriculture, Food Systems, and Nutrition: Meeting the Challenge. Glob. Chall., 1(3), 1600002. DOI: $10.1002 / \mathrm{gch} 2.201600002$ 
13. Carvajal, S.C., Kibor, C., McClelland, D.J., et al. (2014) Stress and sociocultural factors related to health status among US-Mexico border farmworkers. J. Immigr. Minor. Health, 16(6), 11761182. DOI: $10.1007 / \mathrm{s} 10903-013-9853-1$

14. Castaneda, A., Doan, D., Newhouse, D., et al. (2016) Who are the poor in the developing world? Policy Research Working Paper, 7844. World Bank, Washington, DC.

15. Manary, M.J., Muglia, L.J., Vogt, S.K., \& Yarasheski, K.E. (2006) Cortisol and its action on the glucocorticoid receptor in malnutrition and acute infection. Metabolism, 55(4), 550-554. DOI: 10.1016/j.metabol.2005.11.009

16. O'Connor, K. Stoecklin-Marois, M. and Schenker, M.B. (2015) Examining nervios among immigrant male farmworkers in the MICASA Study: sociodemographics, housing conditions and psychosocial factors. J. Immigr. Minor. Health, 17(1), 198-207. DOI: 10.1007/s10903-013-98598

17. Pulgar, C. A., Trejo, G., Suerken, C., et al. (2016). Economic Hardship and Depression Among Women in Latino Farmworker Families. Journal of immigrant and minority health, 18(3), 497504. DOI: $10.1007 / \mathrm{s} 10903-015-0229-6$

18. Tomiyama, A. J., Mann, T., Vinas, D., et al. (2010). Low calorie dieting increases cortisol. Psychosomatic medicine, 72(4), 357-364. DOI: 10.1097/PSY.0b013e3181d9523c

19. Miller, J.V., LeBouf, R.F., Kelly, K.A., et al. (2018) The Neuroinflammatory Phenotype in a Mouse Model of Gulf War Illness is Unrelated to Brain Regional Levels of Acetylcholine as Measured by Quantitative HILIC-UPLC-MS/MS. Toxicol. Sci., 165(2), 302-313. DOI: 10.1093/toxsci/kfy130

20. Caughlan, A., Newhouse, K., Namgung, U., \& Xia, Z. (2004) Chlorpyrifos induces apoptosis in rat cortical neurons that is regulated by a balance between p38 and ERK/JNK MAP kinases. Toxicol. Sci., 78(1), 125-134. DOI: 10.1093/toxsci/kfh038

21. Farkhondeh, T., Mehrpour, O., Buhrmann, C., et al. (2020) Organophosphorus Compounds and MAPK Signaling Pathways. Int. J. Mol. Sci., 21(12), 4258. DOI: 10.3390/ijms21124258

22. Gao, J., Naughton, S. X., Beck, W. D., et al. (2017a) Chlorpyrifos and chlorpyrifos oxon impair the transport of membrane bound organelles in rat cortical axons. Neurotoxicology, 62, 111-123. DOI: $10.1016 /$ j.neuro.2017.06.003

23. Yang, D., Howard, A., Bruun, D., et al. (2008) Chlorpyrifos and chlorpyrifos-oxon inhibit axonal growth by interfering with the morphogenic activity of acetylcholinesterase. Toxicol. Appl. Pharmacol., 228(1), 32-41. DOI: 10.1016/j.taap.2007.11.005

24. Hillmer, E. J., Zhang, H., Li, H. S., \& Watowich, S. S. (2016) STAT3 signaling in immunity. Cytokine Growth Factor Rev., 31, 1-15. DOI: 10.1016/j.cytogfr.2016.05.001

25. Sengupta, T. K., Talbot, E. S., Scherle, P. A., and Ivashkiv, L. B. (1998) Rapid inhibition of interleukin-6 signaling and Stat 3 activation mediated by mitogen-activated protein kinases. PNAS USA, 95(19), 11107-11112. DOI: 10.1073/pnas.95.19.11107 
26. O'Callaghan, J.P. \& Sriram, K. (2004) Focused microwave irradiation of the brain preserves in vivo protein phosphorylation: Comparison with other methods of sacrifice and analysis of multiple phosphoproteins. J. Neurosci. Methods, 135(1-2), 159-168. DOI: 10.1016/j.jneumeth.2003.12.006

27. O'Callaghan, J. P., Brinton, R. E., \& McEwen B. S. (1991) Glucocorticoids regulate the synthesis of glial fibrillary acidic protein in intact and adrenalectomized rats but do not affect its expression following brain injury. J. Neurochem. 57(3), 860-869. DOI: 10.1111/j.14714159.1991.tb08230.x

28. O'Callaghan, J. P., Kelly, K. A., Locker, A. R., Miller, D. B., \& Lasley S. M. (2015) Corticosterone primes the neuroinflammatory response to DFP in mice: potential animal model of Gulf War Illness. J. Neurochem. 133(5), 708-721. DOI: 10.1111/jnc.13088

29. Jiang, W., Duysen, E. G., \& Lockridge, O. (2012) Mice treated with a nontoxic dose of chlorpyrifos oxon have diethoxyphosphotyrosine labeled proteins in blood up to 4 days post exposure, detected by mass spectrometry. Toxicology, 295(1-3), 15-22. DOI: 10.1016/j.tox.2012.03.001

30. Cole, T. B., Walter, B. J., Shih, D. M., et al. (2005) Toxicity of chlorpyrifos and chlorpyrifos oxon in a transgenic mouse model of the human paraoxonase (PON1) Q192R polymorphism. Pharmacogenet. Genomics, 15(8), 589-598. DOI: 10.1097/01.fpc.0000167327.08034.d2

31. Gupta R. C. (2004). Brain regional heterogeneity and toxicological mechanisms of organophosphates and carbamates. Toxicol. Mech. Methods, 14(3), 103-1080. DOI: $10.1080 / 15376520490429175$

32. Stavinoha W. B., Modak A. T., and Weintraub S. T. (1976) Rate of accumulation of acetylcholine in discrete regions of the rat brain after dichlorvos treatment. J. Neurochem., 27(6), 1375-1378. DOI: 10.1111/j.1471-4159.1976.tb02618.x

33. Won Y. K., Liu J., Olivier K.Jr., Zheng Q., and Pope C. N. (2001) Age-related effects of chlorpyrifos on acetylcholine release in rat brain. Neurotoxicology, 22(1), 39-48. DOI: $10.1016 / \mathrm{s} 0161-813 \mathrm{x}(00) 00009-7$

34. Hulse, R.E., Kunkler, P.E., Fedynyshyn, J.P., and Kraig R.P. (2004) Optimization of multiplexed bead-based cytokine immunoassays for rat serum and brain tissue. J. Neurosci. Methods., 136(1), 87-98. DOI: 10.1016/j.jneumeth.2003.12.023

35. Alavanja, M.C., Sprince, N.L., Oliver, E., et al. (2001) Nested case-control analysis of high pesticide exposure events from the Agricultural Health Study. Am. J. Ind. Med., 39(6), 557-563. DOI: $10.1002 /$ ajim. 1054

36. Alavanja, M.C.R. (2010) Pesticides Use and Exposure Extensive Worldwide. Rev. Environ. Health, 24(4), 303-309. DOI: 10.1515/reveh.2009.24.4.303

37. Atwood, D. and Paisley-Jones, C. (2017) Pesticides Industry Sales and Usage 2008-2012 Market Estimates. US Environmental Protection Agency; Washington (DC). Available from: 
https://www.epa.gov/sites/production/files/2017-01/documents/pesticides-industry-sales-usage2016_0.pdf

38. Berridge, M.J., Cobbold, P.H., and Cuthbertson, K.S. (1998) Spatial and Temporal Aspects of Cell Signaling. Philos. Trans. R. Soc. Lond. B. Biol. Sci., 320(1199), 325-343. DOI: 10.1098/rstb.1988.0080

39. Boyd, J.W. \& Neubig, R. (2019) Cellular Signal Transduction in Toxicology and Pharmacology: Data Collection, Analysis, and Interpretation, John Wiley \& Sons, Ltd, Hoboken. ISBN: 978-1-119-06026-0

40. Loo, L.H., Bougen-Zhukov, N.M., \& Tan, W.C. (2017) Early spatiotemporal-specific changes in intermediate signals are predictive of cytotoxic sensitivity to TNF $\alpha$ and co-treatments. Sci. Rep., 7, 43541. DOI: 10.1038/srep43541

41. Chang, F., Steelman, L.S., Lee, J.T., et al. (2003) Signal transduction mediated by the Ras/Raf/MEK/ERK pathway from cytokine receptors to transcription factors: potential targeting for therapeutic intervention. Leukemia, 17(7), 1263-1293. DOI: 10.1038/sj.leu.2402945

42. Geest, C.R., Buitenhuis, M., Groot Koerkamp, M.J., et al. (2009) Tight control of MEK-ERK activation is essential in regulating proliferation, survival, and cytokine production of CD34+derived neutrophil progenitors. Blood, 114(16), 3402-3412. DOI: 10.1182/blood-2008-08-175141

43. Gao, F., Li, F., Miao, Y., et al. (2017b) Involvement of the MEK-ERK/p38-CREB/c-fos signaling pathway in Kir channel inhibition-induced rat retinal Müller cell gliosis. Sci. Rep., 7, 1480. DOI: $10.1038 / \mathrm{s} 41598-017-01557-\mathrm{y}$

44. Steelman, L. S., Chappell, W. H., Abrams, S. L., et al. (2011). Roles of the Raf/MEK/ERK and PI3K/PTEN/Akt/mTOR pathways in controlling growth and sensitivity to therapyimplications for cancer and aging. Aging, 3(3), 192-222. DOI: 10.18632/aging.100296

45. Xing, J., Kornhauser, J. M., Xia, Z., Thiele, E. A., \& Greenberg, M. E. (1998). Nerve growth factor activates extracellular signal-regulated kinase and p38 mitogen-activated protein kinase pathways to stimulate CREB serine 133 phosphorylation. Mol Cell. Biol., 18(4), 1946-1955. DOI: 10.1128/mcb.18.4.1946

46. Biever, A., Valjent, E., \& Puighermanal, E. (2015) Ribosomal Protein S6 Phosphorylation in the Nervous System: From Regulation to Function. Front. Mol. Neurosci., 8, 75. DOI: 10.3389/fnmol.2015.00075

47. McCubrey, J.A., Steelman, L.S., Chappell, W.H., et al. (2007). Roles of the Raf/MEK/ERK pathway in cell growth, malignant transformation and drug resistance. Biochim. Biophys. Acta., 1773(8), 1263-1284. DOI: 10.1016/j.bbamcr.2006.10.001

48. Zhu, X., Dubey, D., Bermudez, C., and Porter, B.E. (2015) Suppressing cAMP response element-binding protein transcription shortens the duration of status epilepticus and decreases the number of spontaneous seizures in the pilocarpine model of epilepsy. Epilepsia, 56(12), 18701878. DOI: $10.1111 /$ epi.13211 
49. Busquets, O., Ettcheto, M., Cano, A., et al. (2020) Role of c-Jun N-Terminal Kinases (JNKs) in Epilepsy and Metabolic Cognitive Impairment. Int. J. Mol. Sci., 21(1), 255. DOI: 10.3390/ijms 21010255

50. Dhanasekaran, D. N. \& Reddy, E. P. (2008). JNK signaling in apoptosis. Oncogene, 27(48), 6245-6251. DOI: 10.1038/onc.2008.301

51. Guma, M. \& Firestein, G.S. (2012) c-Jun N-Terminal Kinase in Inflammation and Rheumatic Diseases. Open Rheumatol. J., 6, 220-231. DOI: 10.2174/1874312901206010220

52. Lazar, S., Egoz, I., Brandeis, R., et al. (2016) Propagation of damage in the rat brain following sarin exposure: Differential progression of early processes. Toxicol. Appl. Pharm. 310, 87-97. DOI: 10.1016/j.taap.2016.09.008.

53. Batista, J. E., Sousa, L. R., Martins, I. K., et al. (2016) Data on the phosphorylation of p38MAPK and JNK induced by chlorpyrifos in Drosophila melanogaster. Data Brief, 9, 32-34. DOI: $10.1016 /$ j.dib.2016.08.033

54. Lee, J.E., Park, J.H., Shin, I.C., and Koh, H.C. (2012) Reactive oxygen species regulated mitochondria-mediated apoptosis in PC12 cells exposed to chlorpyrifos. Toxicol. Appl. Pharm., 263(2), 148-162. DOI: 10.1016/j.taap.2012.06.005

55. Tan, D.H., Peng, S.Q., Wu, Y.L., et al. (2009) Chlorpyrifos induces delayed cytotoxicity after withdrawal in primary hippocampal neurons through extracellular signal-regulated kinase inhibition. Biol. Pharm. Bull., 32(10), 1649-1655. DOI: 10.1248/bpb.32.1649.

56. Salojin, K.V., Zhang, J., \& Delovitch, T.L. (1999) TCR and CD28 are coupled via ZAP-70 to the activation of the Vav/Rac-1-/PAK-1/p38 MAPK signaling pathway. J Immunol., 163(2), 844853.

57. Au-Yeung, B.B., Deindl, S., Hsu, L., et al. (2009) The structure, regulation, and function of ZAP-70. Immunol. Rev., 228(1), 41-57. DOI: 10.1111/j.1600-065X.2008.00753.X

58. Kadlecek, T.A., van Oers, N.S., Lefrancois, L., et al. (1998) Differential Requirements for ZAP-70 in TCR Signaling and T Cell Development. J. Immunol., 161(9), 4688-4694.

59. Kugyelka, R., Prenek, L., Olasz, K., et al. (2019) ZAP-70 Regulates Autoimmune Arthritis via Alterations in T Cell Activation and Apoptosis. Cells, 8(5), 504. DOI: 10.3390/cells8050504.

60. Liu, J., Guo, K., Hu, L., et al. (2019) ZAP70 deficiency promotes reverse cholesterol transport through MAPK/ERK pathway in Jurkat cell. Mol. Immunol., 107, 21-28. DOI: 10.1016/j.molimm.2019.01.001

61. Wang, H., Kadlecek, T.A., Au-Yeung, B.B., et al. (2010) ZAP-70: An Essential Kinase in TCell Signaling. Cold Spring Harb. Perspect. Biol., 2(5), a002279. DOI: 10.1101/cshperspect.a002279 


\section{Chapter 5: Differential phosphoprotein signaling in the cortex in mouse models of Gulf War Illness using corticosterone and acetylcholinesterase inhibitors}

Parts of this chapter have been published previously: Julia A. Penatzer, Julie V. Miller, Nicole Prince, Misa Shaw, Cayla Lynch, Mackenzie Newman, Gerald R. Hobbs, Jonathan W. Boyd (2021) Differential phosphoprotein signaling in the cortex in mouse models of Gulf War Illness using corticosterone and acetylcholinesterase inhibitors. Heliyon, 7, e07552. https://doi.org/10.1016/j.heliyon.2021.e07552

Veterans from the 1990-91 Gulf War were exposed to organophosphate (OP) acetylcholinesterase inhibitors (AChEIs), and, following service, an estimated one-third began suffering from a medically unexplained, multi-symptom illness termed Gulf War Illness (GWI). However, even 30 years after deployment, these veterans continue to suffer from this symptomology and neither the exact etiology, nor a cure has been found. The work presented in the previous two chapters of this dissertation introduce two OP AChEIs, with and without corticosterone (CORT) exposure, that have been validated for this specific disease state of GWI. To further elucidate the mechanisms associated with these mouse models and how they relate to the diverse symptomology of GWI, a deeper investigation into these intracellular responses in the cortex were performed to characterize the early cellular signaling changes associated with this exposure-initiated neuroinflammation. Eleven of the post-translationally modified protein targets from Chapters 3 and 4 were chosen to be further examined. Phosphoprotein responses were found to be exposure specific following AChEI insult, with and without CORT. Specifically, a 3-way interaction displayed a significant interaction (cross) between CORT and AChEI for p-BAD, p-RPS6, p-SRC, p-SYK, and p-JNK (Table 3), indicating that this subset of phosphoproteins play an important role in differentiating between all four exposures. Ingenuity Pathway Analysis further presented the divergence between all exposure groups, especially at the later time points ( 2 and $24 \mathrm{~h}$ post-exposure). The results of 
this study provide insight into differentially activated pathways depending on AChEI in these GWI models.

\subsection{Introduction}

Gulf War Illness (GWI) is a chronic, multi-symptom disorder that has afflicted veterans of the 1990-91 Persian Gulf War (GW) since they left theater. Approximately 30\% of deployed veterans exhibit a variety of symptoms including cognitive and memory impairments, depression, and gastrointestinal disorders [1-7]. The symptoms are consistent with features of "sickness behavior," the basis of which is elaboration of inflammatory mediators in the brain, i.e., neuroinflammation [8-9]. Studies have demonstrated that neuroinflammation is common among GW veterans, and is not confounded by sex, age, or body mass index [10-13], leading to a consensus that neuroinflammation is a hallmark of GWI [7-8].

The exact etiology of GWI remains unknown, but the Research Advisory Committee on Gulf War Veterans' Illnesses concluded that exposure to acetylcholinesterase inhibitors (AChEIs) are the most likely cause of the symptoms of GWI [14-15]; these AChEI exposures include the chemical warfare agent sarin [16], or pesticides, such as chlorpyrifos [17]. Veterans were also exposed to a variety of mental/physical stressors in theater [18]. Therefore, O'Callaghan et al. (2015), established a GWI mouse model using corticosterone (CORT; the rodent surrogate for cortisol) pre-treatment at levels that would be associated with high stress in combination with either diisopropyl fluorophosphate (DFP; sarin surrogate) or chlorpyrifos oxon (CPO; oxon metabolite of chlorpyrifos) [1-2]; these models resulted in marked neuroinflammation characterized as increases in a panel of cytokines and chemokines measured by qPCR. In an effort to elucidate the underlying mechanisms for these observations, Miller et al. (2018), measured acetylcholine concentrations for each model and found that acetylcholinesterase (AChE) inhibition 
is compound specific when pretreated with CORT: CORT ameliorated acetylcholine increase induced by exposure to DFP, but inhibition caused by CPO was not ameliorated by CORT priming [19]. However, both models still resulted in inflammation suggesting an AChE-independent pathway may be the driving force behind the exacerbated neuroinflammation [19].

Cytokine-initiated inflammation, like that observed with these models of neuroinflammation [1-2], directly initiates cellular signaling changes in impacted tissues, which can be measured through post-translational modifications (e.g., protein phosphorylation) at early time points postexposure [20-21]. Protein phosphorylation is vital to the coordination of cellular functions and leads to a cascade of cellular signals; however, abnormal or prolonged phosphorylations can lead to dysregulation of signaling pathways, which is the basis of a number of disease states [22-26]. This study was conducted to distinguish key phosphorylation events that are involved in these pathways to better elucidate the cellular response mechanisms relevant to these acute exposure models of GWI. Moreover, this study was performed to probe the differences in the signaling cascades for these AChEIs, with and without CORT. Using this knowledge, we attempt to understand how these responses ultimately lead to the diverse symptomology of GWI.

In this study, adult male C57BL/6J mice were exposed to CORT $(200 \mu \mathrm{g} / \mathrm{mL})$ in the drinking water for seven days, and given a single intraperitoneal (i.p.) injection of either DFP (4.0 mg/kg) or CPO $(8.0 \mathrm{mg} / \mathrm{kg})$ on the eighth day. Mice were euthanized at $0.5,2$, and $24 \mathrm{~h}$ post-exposure, and 11 post-translational modifications protein targets were measured in the cortex to understand the temporality of phosphoprotein responses in these validated mouse models of GWI.

\subsection{Materials and methods}

\subsubsection{Data description}


Relative phosphoprotein concentrations were measured after exposure to DFP or CPO as described in chapters 3 and 4, respectively. Briefly, mice received CORT in the drinking water (200 $\mu \mathrm{g} / \mathrm{mL}$ in $1.2 \%$ ethanol) for seven days prior to exposure. Control groups received $1.2 \%$ ethanol water. On day eight, mice were given a single i.p. injection of saline (0.9\%), peanut oil (CPO vehicle), DFP $(4.0 \mathrm{mg} / \mathrm{kg})$, or CPO $(8.0 \mathrm{mg} / \mathrm{kg})$ in the morning and returned to their home cage. Mice were euthanized at three time points $(0.5,2$, and $24 \mathrm{~h}$ post-exposure) by focused microwave irradiation to provide a snapshot of the early cell signaling alterations that result in long-term impacts using a high-throughput approach. All procedures were performed under protocols approved by the Institutional Animal Care and Use Committee of the Centers for Disease Control and Prevention, National Institute for Occupational Safety and Health, and the animal facility was certified by the American Association for Accreditation of Laboratory Animal Care.

Samples were assayed using the Bio-Plex Pro multiplexed magnetic bead-based immunoassay reagent kit for the following 11 targets phosphorylated at the indicated amino acid residues: p-BAD (S136), p-S6 Ribosomal P (S235/S236; p-RPS6), p-SRC (Y416), p-SYK (Y352), p-CREB (S133), p-ERK1/2 (T202/Y204, T185/Y187), p-GSK3 $\alpha / \beta$ (S21/S9), p-IкB $\alpha$ (S32/S36), p-JNK (T183/Y185), p-MEK1/2 (S217/S221), and p-p90RSK (S380). Samples were analyzed using a Bio-Plex 200 suspension array system and Pro II Wash Station (Bio-Rad), according to the manufacturer's instructions.

\subsubsection{Data processing}

Relative phosphorylation was determined for each target. The averaged raw values were blank-subtracted, and any phosphoprotein non-detects (response was less than blank) were removed before analysis. The blank-subtracted values were then normalized to the respective controls (saline for DFP, peanut oil for CPO) at each time point. A Grubbs' test was performed to 
remove any significant outliers $(\alpha=0.05)$. As previously mentioned, any non-surviving animals were also excluded from analysis. Thus, these analyses resulted in at least $\mathrm{N}=4$ animals for all phosphoproteins. Data analyses were performed using GraphPad Prism (V5; San Diego, CA, USA) or SAS JMP (V14; Cary, NC, USA). The normalized phosphorylation \pm the standard error of the mean (SEM) for each exposure and time point were used to identify significant targets.

\subsubsection{3-way interaction}

A 3-way interaction was conducted using SAS JMP to determine the main effects and interactions for each phosphoprotein [27]. The factors for the 3-way interaction were time $(0.5,2$, $24 \mathrm{~h}$ ), and exposure (CORT effect or AChEI effect). A threshold of $\mathrm{p}<0.05$ was chosen, and a Bonferroni correction (for the seven tests) was applied to omit a type I error [28-29], resulting in a significance level of $\mathrm{p}<0.007$ representing findings that were statistically significant. A 3-way interaction splits the exposures into binary effects of CORT (CORT+DFP and CORT+CPO versus DFP and CPO alone) or AChEI (CPO alone and CORT+CPO versus DFP alone and CORT+DFP), which allows for differences to be easily detected between groups. Cube plots were used to understand the nature of the relationships between the 3-way interactions [30].

\subsubsection{Ingenuity Pathway Analysis (IPA)}

The relative phosphorylation responses were further evaluated using QIAGEN's Ingenuity Pathway Analysis (IPA; Redwood City, CA, USA) to understand the network and cellular pathway interactions of the selected phosphoproteins. The proposed networks were generated for all three time points $(0.5,2$, and $24 \mathrm{~h})$ following each AChEI exposure with and without CORT (DFP, CPO, CORT+DFP, CORT+CPO). All of the networks were comprised of the experimental dataset in addition to other projected nodes likely to be involved in the network based on the observations from the experimental dataset and Ingenuity's knowledge base. IPA also assigned a p-score 
(negative $\log 10$ (p-value of Fisher's exact test)) to each of the networks. For more information

$\begin{array}{llll}\text { refer } & \text { to } & \text { Ingenuity's }\end{array}$

(http://pages.ingenuity.com/rs/ingenuity/images/0812\%20upstream_regulator_analysis_whitepap

er.pdf).

\subsection{Results}

A panel of phosphoproteins involved in regulatory stress and inflammatory pathways associated with early responses of neuroinflammation were assayed via multiplex bead-based ELISA. The phosphoprotein responses were normalized to controls (saline or peanut oil for DFP and $\mathrm{CPO}$, respectively) in the cortex at $0.5,2$, and $24 \mathrm{~h}$ post-exposure. As previously mentioned, the DFP-exposure cohort resulted in all saline $(\mathrm{N}=5)$, CORT alone $(\mathrm{N}=5)$, and CORT+DFP $(\mathrm{N}=7)$ exposed mice surviving at the $24 \mathrm{~h}$ time point, but only 5 of 14 mice from the DFP exposure alone group surviving (36\% survival rate). Conversely, for the CPO exposure cohort: all of the peanut oil $(\mathrm{N}=5)$, CORT $(\mathrm{N}=5)$, and $\mathrm{CPO}(\mathrm{N}=7)$ exposed mice survived all the time points, but only 5 of 7 mice from the CORT+CPO exposure survived at the $2 \mathrm{~h}$ and $24 \mathrm{~h}$ time points $(71 \%$ survival rate).

\subsubsection{3-way interactions revealed distinct phosphorylation responses}

To explore the potential interactions between CORT and AChEIs and how time may affect these interactions, a 3-way interaction was performed on all of the phosphoprotein targets. A 3way interaction allows us to further determine if the significant effects associated with each target are driven by the different exposures (AChEI or CORT), time, or both. The factors tested were time $(0.5,2,24 \mathrm{~h})$, and exposure (CORT effect or AChEI effect), and a level of $\mathrm{p}<0.007$ was used to determine significance after a Bonferroni correction. The 3-way interaction revealed p-RPS6, 
p-CREB, p-ERK1/2, p-IкB $\alpha$, and p-JNK all were significant for time alone, demonstrating the importance of monitoring the phosphoprotein responses over time (Table 5.1).

Table 5.1. 3-way interaction revealed distinct phosphoprotein responses in mouse models of Gulf War Illness. A 3way interaction was conducted using SAS JMP to determine significant interactions for all 11 phosphoproteins. The factors were time $(0.5,2,24 \mathrm{~h})$ and exposure (CORT effect or AChEI effect). A significance level of $\mathrm{p}<0.007$ was used to determine statistical significance. Only interactions with statistical significance are shown for each phosphoprotein. A " $\mathrm{x}$ " is used to display a cross between two factors.

\begin{tabular}{|c|c|c|}
\hline Phosphoprotein & Interaction & p-value \\
\hline \multirow{3}{*}{ p-BAD (S136) } & CORT x AChEI & 0.00007 \\
\hline & CORT & 0.00010 \\
\hline & Time & 0.00545 \\
\hline \multirow{2}{*}{ p-RPS6 (S235/S236) } & Time & 0.00066 \\
\hline & CORT x AChEI & 0.00498 \\
\hline \multirow{4}{*}{ p-SRC (Y416) } & Time $\mathrm{x}$ AChEI & 0.00001 \\
\hline & CORT x AChEI & 0.00021 \\
\hline & AChEI & 0.00379 \\
\hline & Time $x$ CORT x AChEI & 0.00642 \\
\hline p-SYK (Y352) & CORT x AChEI & 0.00247 \\
\hline p-CREB (S133) & Time & 0.00058 \\
\hline \multirow{4}{*}{$\begin{array}{l}\text { p-ERK1/2 } \\
\text { T185/Y187) }\end{array}$} & Time & 0.00000 \\
\hline & AChEI & 0.00000 \\
\hline & Time $\mathrm{x}$ AChEI & 0.00001 \\
\hline & Time $\mathrm{x}$ CORT & 0.00017 \\
\hline
\end{tabular}




\begin{tabular}{|l|l|l|}
\hline \multirow{2}{*}{$\mathbf{p - G S K 3} \boldsymbol{\alpha} / \mathbf{\beta}(\mathbf{S 2 1} / \mathbf{S 9})$} & AChEI & 0.00004 \\
\cline { 2 - 3 } & CORT & 0.00117 \\
\hline $\mathbf{p}-$ IKB $\boldsymbol{\alpha}(\mathbf{S 3 2} / \mathbf{S 3 6})$ & Time & 0.00006 \\
\hline \multirow{2}{*}{$\mathbf{p - J N K ~ ( T 1 8 3 / Y 1 8 5 ) ~}$} & Time x CORT x AChEI & 0.00109 \\
\cline { 2 - 3 } & Time & 0.00440 \\
\hline
\end{tabular}

Of the 11 phosphoproteins tested, 6 of the targets (p-BAD, p-RPS6, p-SRC, p-SYK, pERK1/2, and p-GSK3 $\alpha / \beta)$ displayed a significant $(\mathrm{p}<0.007)$ interaction between CORT, AChEI, or the cross (CORT x AChEI) (Table 5.1). A significant interaction for CORT represents a difference between the primed with CORT AChEI exposures (CORT+DFP, CORT+CPO) versus those without CORT (DFP, CPO). A significant AChEI interaction differentiates between the AChEI exposures regardless of CORT (CORT+DFP and DFP versus CORT+CPO and CPO). Our results indicate $\mathrm{p}-\mathrm{BAD}$ and $\mathrm{p}-\mathrm{GSK} 3 \alpha / \beta$ were significant for the CORT effect demonstrating a significant difference $(\mathrm{p}<0.007)$ between the CORT primed exposures (i.e., CORT+DFP and CORT+CPO) from the AChEIs alone (DFP and CPO) (Table 5.1). The significant CORT interaction provides further evidence of how the mixtures of CORT+AChEIs impact the phosphorylation of specific proteins, and therefore distinguishes from the pathways that are affected by AChEIs alone. Phosphorylated SRC, ERK1/2, and GSK $3 \alpha / \beta$ were significant $(p<0.007)$ for the AChEI interaction, which indicates these specific phosphoproteins are important to differentiate between the inhibitor exposures regardless of the addition of CORT. A significant cross $(\mathrm{p}<0.007)$ between CORT and AChEI was observed for $\mathrm{p}-\mathrm{BAD}, \mathrm{p}-\mathrm{RPS6}$, $\mathrm{p}-\mathrm{SRC}$, and $\mathrm{p}$ SYK (Table 5.1), indicating there was a significant difference between each of the exposures and both interactions attribute to the differences between the four exposures (DFP, CORT+DFP, CPO, 
CORT+CPO). Thus, these phosphoproteins may be good indicators to differentiate between all the exposures. Phosphorylated JNK and SRC demonstrated a significant cross $(\mathrm{p}<0.007)$ between all factors (Time x CORT x AChEI) (Table 5.1; Figure 5.1), displaying that both p-SRC and p-JNK can also differentiate between all the exposures, but in a time dependent manner.
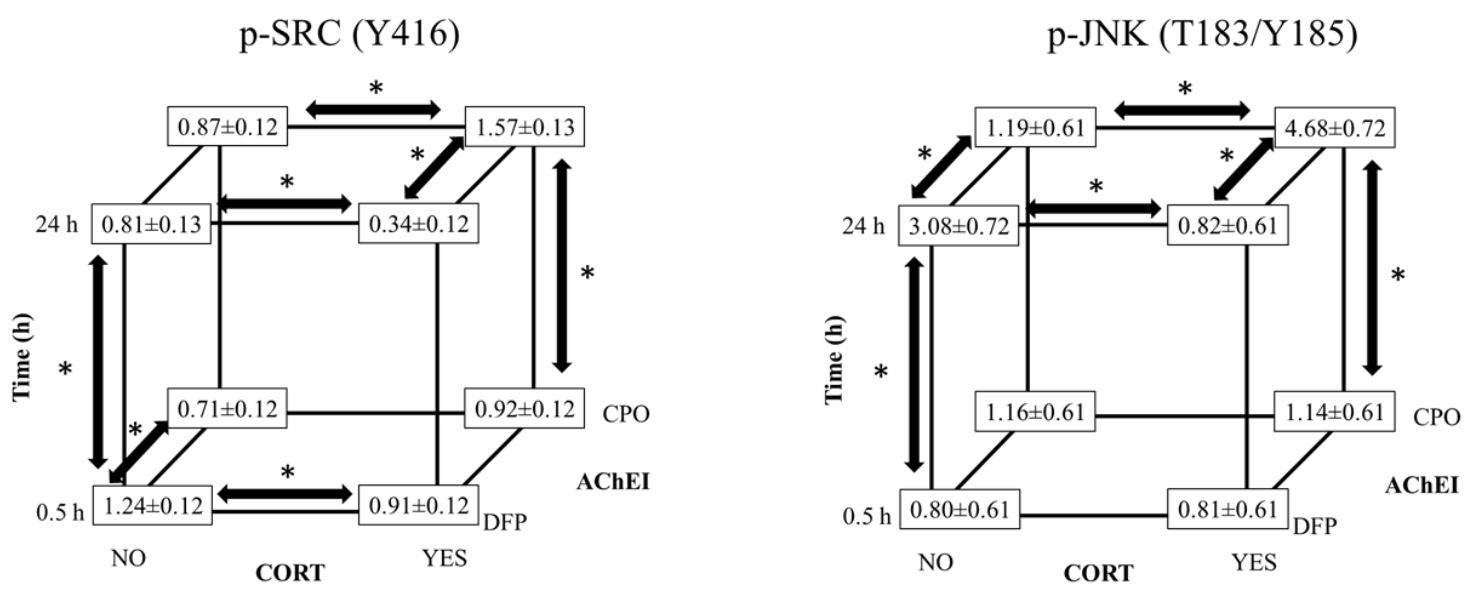

Figure 5.1. Cube plots for p-SRC (Y416) and p-JNK (T183/Y185). A 3-way interaction was conducted using SAS JMP to determine the main effects and interactions for each phosphoprotein. The factors were time $(0.5,2,24 \mathrm{~h})$ and exposure (CORT effect or AChEI effect). Cube plots were used to understand the nature of the relationships between the three-factor interactions. The vertices display the fitted mean of the logarithm transformed data \pm standard error. Only interactions with a statistically significant 3 -way cross are shown $(\mathrm{p}<0.007)$, where arrows with * represent the significant differences between the interactions.

\subsubsection{Ingenuity Pathway Analysis (IPA) generated networks}

IPA was performed to further explore the pathways and biological processes associated with the tested phosphoprotein responses, while identifying additional nodes of interest for future studies. The normalized phosphorylation responses measured in this study were used to generate a network with a total of 35 nodes, 11 of which are from the dataset, but the remaining 24 are based on connectivity with the dataset and known pathways from literature (Table 5.2; Supplemental Tables 1-3) [31]. At $0.5 \mathrm{~h}$, over half of the nodes generated were the same regardless 
of exposure (Table 5.2). However, at 2 and $24 \mathrm{~h}$ there were only six and four nodes, respectively, that were similar between exposures demonstrating divergence of these networks at later time points. Specifically, at $2 \mathrm{~h}$ post-exposure to DFP alone, 14 of the generated nodes were not found in the other networks (Supplemental Table 2). At $24 \mathrm{~h}$, the CORT+DFP network generated 20 unique nodes to that exposure (Supplemental Table 3). Amongst all of the generated networks, two cytokines, tumor necrosis factor (TNF) and interferon gamma (IFN- $\gamma$ ), a kinase, PDPK1, and a phosphatase, PTEN, were observed across all of the time points and exposures (Table 5.2). Therefore, these time-independent common nodes are highly connected to the dataset, and may be shared links to the observed neuroinflammation with both of these AChEIs [1-2, 19, 32].

Table 5.2: IPA projected nodes. IPA networks were generated from the experimental dataset and the top molecules in these pathways were reported. The table represents the nodes that were similar across all exposures at each time point. The network p-score (negative $\log 10$ (p-value of Fisher's exact test)) for all networks was 28.

\begin{tabular}{|l|l|l|}
\hline $0.5 \mathrm{~h}$ & $2 \mathrm{~h}$ & $24 \mathrm{~h}$ \\
\hline TNF, PDPK1, IFN- $\gamma$, PTEN, & & \\
ALDH1L1, FGR, BTG1, CRK, & TNF, PDPK1, IFN- $\gamma$, PTEN, & TNF, PDPK1, IFN- $\gamma$, PTEN \\
HAVCR2, RPS6KA3, RASGRF2, & ALDH1L1, FGR & \\
ATE1, GPR19, TRAFD1, & & \\
PPP2R1A & & \\
\hline
\end{tabular}

\subsection{Discussion}

A complete understanding of the exposures that led to GWI, as well as viable treatments, have yet to be determined 30 years after the 1990-91 GW. Previous research suggests that exposure to AChEIs, such as sarin or pesticides (e.g., chlorpyrifos), are one of the probable causes of GWI $[8,17,33]$. With this in mind, several rodent models have investigated the role of AChEI exposure, specifically DFP or CPO, in GWI, and related this to significant neurological effects [34-39]. To build upon the likely exposure to high stress, and better replicate in-theater conditions, rodent 
models have included exogenous CORT priming in combination with these AChEIs [1-2, 19]. Studies utilizing these rodent models of GWI have demonstrated exacerbated neuroinflammation in CORT pretreated animals compared to the controls, which has been related to the sickness behavior observed in veterans from the GW [1-2]. Conversely, Miller et al. (2018), displayed differences in AChE inhibition depending on the AChEI (CORT ameliorated acetylcholine increase caused by DFP, but AChE inhibition caused by CPO was not impacted by CORT) [19]. These results suggest a potential AChE-independent route of GWI-related neuroinflammation. To better understand the neurological responses associated with these mouse models of GWI, this study investigated early intracellular signaling responses via phosphoprotein analysis in the cortex.

As previously stated in Chapter 4, our results indicated, CORT+CPO led to significant increases $(\mathrm{p}<0.05)$ in phosphorylation of proteins in the MEK/ERK cascade at the early time points (0.5 and $2 \mathrm{~h}$ ) (Figure 4.2). Conversely, at $24 \mathrm{~h}$ post-exposure to CORT+CPO, the shift in MAPK signaling occurs with $\mathrm{p}-\mathrm{JNK}$ increasing in response to CORT+CPO, while $\mathrm{p}-\mathrm{MEK} 1 / 2$ and $\mathrm{p}$ ERK1/2 phosphorylation decreases (Figure 4.2). Within the present study, the 3-way interaction analysis showed a significant $(\mathrm{p}<0.007)$ response for time alone for several proteins involved in MAPK signaling (p-RPS6, p-CREB, p-ERK1/2, and p-JNK; Table 5.1), further indicating the temporal differences in this pathway. All of the temporal responses taken together indicate that early inflammation associated with $\mathrm{CORT}+\mathrm{CPO}$ involves MAPK proteins that transition from MEK/ERK (pro-survival) to JNK (proapoptotic) in less than $24 \mathrm{~h}$ post-exposure. Similar to CORT+CPO, JNK was also significantly phosphorylated $(\mathrm{p}<0.05)$ at $24 \mathrm{~h}$ following DFP alone exposure (Figure 3.2), but the combination of CORT+DFP ameliorated this significant phosphoprotein response as shown in Chapter 3. 
To expand on the differences in phosphoprotein responses observed in the two-way analysis of variances (ANOVAs) to these AChEIs, with and without CORT, we observed significant crosses $(\mathrm{p}<0.007)$ from the 3-way interaction. Interestingly, p-BAD, p-RPS6, p-SRC, and p-SYK (Table 5.1; Figure 5.1) all displayed a significant cross between CORT and AChEI, indicating that this subset phosphoproteins also play an important role in differentiating between all four exposures (DFP, CPO, CORT+DFP, CORT+CPO). Importantly, these phosphoproteins connect previous discussions of $\mathrm{p}-\mathrm{BAD}, \mathrm{p}-\mathrm{SRC}$, and $\mathrm{p}-\mathrm{SYK}$ that have crucial relationships that link to the MAPK signaling pathways explored in this study [40-41]. Additionally, p-JNK, which has been extensively discussed throughout this dissertation, displayed a significant cross between all three factors (Figure 5.1) indicating the ability to differentiate between all four exposures, but in a time-dependent manner. Previously-reported increases in cytokine responses (e.g., TNF- $\alpha$, IL1) from $6 \mathrm{~h}$ post-exposure to CORT+AChEIs may be leading to the significant differences associated with p-JNK appear at 24 h post-exposure [2]. Moreover, these significant increases in p-JNK at $24 \mathrm{~h}$ aligns with the animal deaths observed at the $24 \mathrm{~h}$ time points for DFP alone and CORT+CPO. Overall, these proteins give us a framework for a better understanding of the pathway divergence associated with each exposure and may offer insight into an AChEindependent neuroimmune mechanism of GWI.

IPA was performed to further explore the pathways associated with the tested phosphoprotein responses. IPA analysis also displayed the divergence between the exposure groups, especially at the later time points ( 2 and $24 \mathrm{~h}$ post-exposure; Table 5.2 ). These distinctions in phosphoprotein responses, depending on AChEI, may be related to the differences Miller et al. (2018) observed in ACh concentrations and relate to the differences observed in the two-way ANOVAs [19]. Importantly, IPA analysis also indicated TNF and IFN- $\gamma$ are common nodes 
between all the exposure networks, independent of time (Table 5.2). Therefore, these timeindependent nodes are highly connected to the dataset, and may be shared links to the observed neuroinflammation with both of these AChEIs [2, 19, 32].

\subsection{Conclusion}

Mouse models of GWI were used to explore early cellular signaling changes in the cortex post-exposure to two AChEIs, with and without CORT. These exposures have been shown to cause neuroinflammation [1-2], but AChE inhibition was AChEI-specific [19]. Here we build upon the previously mentioned work in which we found $\mathrm{CORT}+\mathrm{CPO}$ appears to be initiating a cell death response via MAPK signaling, indicated by increases in p-JNK, and decreases in p-ERK1/2 and p-MEK1/2. Similarly, DFP activates p-JNK, but CORT priming ameliorates this response. In the present chapter, we report a subset of phosphoproteins are also activated in an AChEI-dependent manner. p-BAD, p-RPS6, p-SRC, p-JNK, and p-SYK all displayed a significant interaction between CORT and AChEI, indicating these phosphoproteins are key in differentiating between all four exposures (DFP, CPO, CORT+DFP, CORT+CPO). Further, IPA analysis verified a divergence of network signaling associated with each exposure by 2 and $24 \mathrm{~h}$ post-exposure. Finally, TNF and IFN- $\gamma$ were found to be crucial nodes for all networks, independent of time or exposure linking to observed inflammation. Overall, this study found an array of inflammationactivated pathways that may provide insight into the very diverse symptomology observed with GWI. Further investigation of the long-term effects of these signal transduction pathways should be conducted to understand the interrelationship of neuroinflammation, acetylcholinesterase inhibition, and phosphoprotein pathways in the disease progression of GWI.

\subsection{Acknowledgements}


The authors gratefully acknowledge James P. O'Callaghan, Kimberly A. Kelly, Lindsay T. Michalovicz, Brenda K. Billig, Christopher M. Felton, Ali Yilmaz, and Fang Ma for assistance with dosing the mice and dissecting the brains. The authors would also like to thank Suzanne Danley for her editorial suggestions. Opinions, interpretations, conclusions, and recommendations are those of the author and are not necessarily endorsed by the Department of Defense. This work was supported by Congressionally Directed Medical Research Programs: Gulf War Illness Research Program Grants (GW170081). 


\subsection{References}

1. O’Callaghan, J.P., Kelly, K.A., Locker, A.R., et al. (2015) Corticosterone primes the neuroinflammatory response to DFP in mice: potential animal model of Gulf War Illness. J. Neurochem., 133(5), 708-21. DOI: 10.1111/jnc.13088

2. Locker, A.R., Michalovicz, L.T., Kelly, K.A., et al. (2017) Corticosterone primes the neuroinflammatory response to Gulf War Illness-relevant organophosphates independently of acetylcholinesterase inhibition. J. Neurochem., 142(3), 444-55. DOI: 10.1111/jnc.14071

3. White, R.F., Steele, L., O'Callaghan, J.P., et al. (2016) Recent research on Gulf War illness and other health problems in veterans of the 1991 Gulf War: Effects of toxicant exposures during deployment. Cortex, 74, 449-475. DOI: 10.1016/j.cortex.2015.08.022

4. Haley, R.W., Kurt, T.L., \& Hom, J. (1997) Is there a Gulf War Syndrome? Searching for syndromes by factor analysis of symptoms. JAMA, 277(3), 215-22.

5. O’Callaghan, J.P., Michalovicz, L.T., \& Kelly, K.A. (2016) Supporting a neuroimmune basis of Gulf War Illness. E. Bio. Medicine, 13, 5-6. DOI: 10.1016/j.ebiom.2016.10.037

6. U.S. Department of Veterans Affairs Public Health (2017) Gulf War Veterans' Medically Unexplained Illnesses, https://www.publichealth.va.gov/exposures/gulfwar/medicallyunexplained-illness.asp (accessed 26 February 2018).

7. Department of Veterans Affairs (2015) Annual Summary of Federally Sponsored Research on Gulf War Veterans' Illnesses for 2015, Washington, D.C.

8. Michalovicz, L. T., Kelly, K. A., Sullivan, K., \& O'Callaghan, J. P. (2020). Acetylcholinesterase inhibitor exposures as an initiating factor in the development of Gulf War Illness, a chronic neuroimmune disorder in deployed veterans. Neuropharmacology, 171, 108073. DOI: 10.1016/j.neuropharm.2020.108073

9. Steele, L., Sastre, A., Gerkovich, M.M., \& Cook, M.R., (2012) Complex factors in the etiology of Gulf War illness: wartime exposures and risk factors in veteran subgroups. Environ. Health Perspect. 120, 112-118. DOI: 10.1289/ehp.1003399

10. Johnson, G. J., Slater, B. C., Leis, L. A., Rector, T. S., \& Bach, R. R. (2016). Blood Biomarkers of Chronic Inflammation in Gulf War Illness. PloS one, 11(6), e0157855. DOI: 10.1371/journal.pone.0157855

11. Whistler, T., Fletcher, M. A., Lonergan, W., et al. (2009). Impaired immune function in Gulf War Illness. BMC Med. Genomics, 2, 12. DOI: 10.1186/1755-8794-2-12

12. Alshelh, Z., Albrecht, D.S., Bergan, C., et al. (2020) In-vivo imaging of neuroinflammation in veterans with Gulf War illness. Brain Behav. Immun., 87, 498-507. DOI: 10.1016/j.bbi.2020.01.020

13. Helmer, D. A., Van Doren, W. W., Litke, D. R., et al. (2020). Safety, Tolerability and Efficacy of Dietary Supplementation with Concord Grape Juice in Gulf War Veterans with Gulf War Illness: 
A Phase I/IIA, Randomized, Double-Blind, Placebo-Controlled Trial. Int. J. Environ. Res. Public Health, 17(10), 3546. DOI: 10.3390/ijerph17103546

14. J. Research Advisory Committee on Gulf War Veterans' Illnesses (2008) Gulf War Illness and the Health of Gulf War Veterans: Scientific Findings and Recommendations, Washington, D.C.

15. Brimfield, A.A. (2012) Chapter Seven - Chemicals of Military Deployments: Revisiting Gulf War Syndrome in Light of New Information. Prog. Mol. Biol. Transl., 112, 209-30. DOI: 10.1016/B978-0-12-415813-9.00007-6

16. Smith, T.C., Gray, G.C., Weir, J.C., et al. (2003) Gulf War veterans and Iraqi nerve agents at Khamisiyah: postwar hospitalization data revisited. Am. J. Epidemiol., 158(5), 457-67. DOI: 10.1093/aje/kwg178

17. Choi, K., Joo, H., Rose, R.L., \& Hodgson, E. (2006) Metabolism of Chlorpyrifos and Chlorpyrifos Oxon by human hepatocytes. J. Biochem. Mol. Toxicol., 20(6), 279-291. DOI: $10.1002 /$ jbt.20145

18. Sapolsky, R.M., Krey, L.C., \& McEwen, B.S. (1985) Prolonged glucocorticoid exposure reduces hippocampal neuron number: implications for aging. J. Neurosci., 5(5), 1222-1227. DOI: 10.1523/JNEUROSCI.05-05-01222.1985

19. Miller, J.V., LeBouf, R.F., Kelly, K.A., et al. (2018) The Neuroinflammatory Phenotype in a Mouse Model of Gulf War Illness is Unrelated to Brain Regional Levels of Acetylcholine as Measured by Quantitative HILIC-UPLC-MS/MS. Toxicol. Sci., 165(2), 302-313. DOI: 10.1093/toxsci/kfy130

20. Han, A.A., Currie, H.N., Loos, M.S., et al. (2016) Spatiotemporal phosphoprotein distribution and associated cytokine response of a traumatic injury. Cytokine, 79, 12-22. DOI: 10.1016/j.cyto.2015.12.006

21. Boyd, J.W. \& Neubig, R. (2019) Cellular Signal Transduction in Toxicology and Pharmacology: Data Collection, Analysis, and Interpretation, John Wiley \& Sons, Ltd, Hoboken.

22. Ardito, F., Giuliani, M., Perrone, D., Troiano, G., \& Muzio, L.L. (2017) The crucial role of protein phosphorylation in cell signaling and its use as targeted therapy. Int. J. Mol. Med., 40(2), 271-280. DOI: 10.3892/ijmm.2017.3036

23. Dhillon, A.S., Hagan, S., Rath, O. \& Kolch, W. (2007) MAP kinase signalling pathways. Oncogene, 26, 3279-3290. DOI: 10.1038/sj.onc.1210421

24. Cohen, P. (2001) The role of protein phosphorylation in human health and disease. Eur. J. Biochem., 268(19), 5001-5010. DOI: 10.1046/j.0014-2956.2001.02473.x

25. Takeuchi, T. \& Abe, T. (1998) Tyrosine phosphorylated proteins in synovial cells of rheumatoid arthritis. Int. Rev. Immunol., 17(5-6), 365-381. DOI: 10.3109/08830189809054411 
26. Lee, J.K. \& Kim, N.J. (2017) Recent Advances in the Inhibition of p38 MAPK as a Potential Strategy for the Treatment of Alzheimer's Disease. Molecules, 22(8), 1287. DOI: $10.3390 /$ molecules 22081287

27. Tavakkolkhah, P., Zimmer, R., \& Kuffner, R. (2018) Detection of network motifs using threeway ANOVA. PLOS One, 13(8), e0201382. DOI: 10.1371/journal.pone.0201382

28. Armstrong, R.A. (2014) When to use the Bonferroni correction. Ophthalmic. Physiol. Opt., 34(5), 502-508. DOI: 10.1111/opo.12131

29. Perneger, T.V. (1998) What's wrong with Bonferroni adjustments. BMJ, 316(7139), 12361238. DOI: $10.1136 / \mathrm{bmj} .316 .7139 .1236$

30. Box, G.E.P., Hunter, J.S., \& Hunter, W.G. (1978) Statistics for Experimenters: Design, Innovation, and Discovery, 2nd Edition, John Wiley \& Sons, Ltd, Hoboken.

31. Ingenuity Pathway Analysis. (October 2015) IPA Training: Maximizing the Biological Interaction of Gene, Transcript, \& Protein Expression Data with IPA. https://chhe.research.ncsu.edu/wordpress/wp-content/uploads/2015/10/IPA-Data-Analysistraining-slides-2016_04.pdf

32. Trageser, K.J., Sebastian-Valverde, M., Naughton, S.X., \& Pasinetti, G.M. (2020) The Innate Immune System and Inflammatory Priming: Potential Mechanistic Factors in Mood Disorders and Gulf War Illness. Front. Psychiatry, 11, 704. DOI: 10.3389/fpsyt.2020.00704

33. Cao, J., Varnell, A., \& Cooper, D. (2011) Gulf War Syndrome: A role for organophosphate induced plasticity of locus coeruleus neurons. Nat. Prec. https://doi.org/10.1038/npre.2011.6057.1

34. Ojo, J.O., Abdullah, L., Evans, J., et al. (2014) Exposure to an organophosphate pesticide, individually or in combination with other Gulf War agents, impairs synaptic integrity and neuronal differentiation, and is accompanied by subtle microvascular injury in a mouse model of Gulf War agent exposure. Neuropathology, 34 (2), 109-127. DOI: 10.1111/neup.12061

35. Hernandez, C.M., Beck, W.D., Naughton, S.X., et al. (2015) Repeated exposure to chlorpyrifos leads to prolonged impairments of axonal transport in the living rodent brain. Neurotoxicology, 47, 17-26. DOI: 10.1016/j.neuro.2015.01.002

36. Nutter, T.J., Johnson, R.D., \& Cooper, B.Y., (2015) A delayed chronic pain like condition with decreased Kv channel activity in a rat model of Gulf War Illness pain syndrome. Neurotoxicology, 51, 67-79. DOI: 10.1016/j.neuro.2015.09.010

37. Cooper, B.Y., Johnson, R.D., \& Nutter, T.J. (2016) Exposure to Gulf War Illness chemicals induces functional muscarinic receptor maladaptations in muscle nociceptors. Neurotoxicology, 54, 99-110. DOI: 10.1016/j.neuro.2016.04.001

38. Cooper, B.Y., Flunker, L.D., Johnson, R.D., \& Nutter, T.J. (2018) Behavioral, cellular and molecular maladaptations covary with exposure to pyridostigmine bromide in a rat model of gulf war illness pain. Toxicol. Appl. Pharamcol., 352, 119-131. DOI: 10.1016/j.taap.2018.05.023 
39. Flunker, L.K., Nutter, T.J., Johnson, R.D., \& Cooper, B.Y. (2017) DEET potentiates the development and persistence of anticholinesterase dependent chronic pain signs in a rat model of Gulf War Illness pain. Toxicol. Appl. Pharmacol., 316, 48-62. DOI: 10.1016/j.taap.2016.12.014

40. Kanie, T., Abe, A., Matsuda, T., et al. (2004) TEL-Syk fusion constitutively activates PI3K/AKT, MAPK and JAK2-independent STAT5 signal pathways. Leukemia, 18, 548-555. DOI: 10.1038/sj.leu.2403266

41. Carnevale, J., Ross, L., Puissant, A., et al. (2013) SYK regulates mTOR signaling in AML. Leukemia, 27(11), 2118-2128. DOI: 10.1038/leu.2013.89 


\section{Chapter 6: Conclusions and Future Directions}

There are several topics addressed within this dissertation with a focus on early signaling that take place to protect and maintain optimal function within the body following exposure to stressors. Further this dissertation displays systemic (Chapter 2) versus localized (Chapters 3-5) responses, as well as different biological samples (saliva vs ex vivo tissue) to analyze various biomarkers of stress. The knowledge obtained from this work provides an opportunity to enhance our understanding of the biochemical adaptations that take place in response to both social and occupational stressors in complex situations. The first study involves the complexity associated with human work, especially those contributing to team dynamics, while the in vivo work provides an insight into a more realistic mixture exposure that may occur in humans and further relates this model to a potential disease state. Despite the information obtained from each of the studies, there are limitations and future work that needs to be completed to further each project.

\subsection{Salivary cytokines and social stress}

Chapter 2 demonstrates that stressors can originate from social or physical situations and can be measured systemically using noninvasive techniques. The $\mathrm{Zephyr}^{\mathrm{TM}}$ bioharness provides real-time measurements of the physiological response experienced during the missions. Importantly, saliva can be used as a biomonitoring technique to measure cytokine response. This

study, discussed in Chapter 2, provides a better understanding of social stress experienced during a physical exercise, especially for military personnel in a naturalistic setting.

\subsubsection{Sample size}

As previously mentioned, a major limitation of this study involves the limited sample size. Despite meeting statistical power, increased samples would provide more accurate averages and 
help determine any potential outliers that may be skewing the data. Furthermore, the subjects in this study were a primarily homogenous samples in terms of age, body mass index, and gender (Table 2.1). While homogenous samples do have advantages when focusing on a specific demographic, these samples often fail to extrapolate the results to the general population. Therefore, to further this work, potential differences observed among genders, socioeconomic, groups, etc. should be included in future studies. Additionally, further investigations would expand to include other teams (e.g., athletics) to further understand the cytokine responses in different physical activity settings.

\subsubsection{Order of missions}

An additional limitation to highlight includes the order of the missions. Again, due to the small sample size, we only performed the experiments in a single manner/order (baseline, success, failure). To help further demonstrate the findings in the study are representative of cytokine increases in response to failure, further studies should randomly assign the order of success and failure between individual missions. Moreover, the team missions followed the individual missions so it may be suggested the cytokine responses are not seen in the team missions because the individuals were able to forecast what may be happening. Therefore, with an increased sample size, future work should also randomly assign whether individuals complete the mission in a team or as individuals first to address this question.

\subsubsection{Correlation to blood}

Another limitation to Chapter 2 involves the lack of correlation to blood cytokine levels. Blood continues to be the standard diagnostic fluid, but there are limitations in the amount of blood that can be drawn from an individual in a single day. In the present study, samples could only be 
drawn from the subjects before the baseline mission and after the failed mission. Therefore, the current studied lacked the ability to perform statistical analysis to determine if blood cytokine levels increased (or decreased) in response to success/failure or significantly correlated to the salivary cytokines. Previous researchers have observed significant correlations between blood and salivary biomarkers during both physical and psychological stress activities, but to further the present work, future studies should be completed to determine if there are any significant correlations to blood biomarkers.

\subsection{Phosphoprotein signaling post-corticosterone and organophosphates exposure}

Chapters 3-4 involve the localized spatiotemporal response to the combination of stress (CORT) with occupational stressors (OPs) following a validated mouse model. It was determined that CORT serves a partially protective role in phosphoprotein signaling post-exposure to DFP; conversely, the combination of CORT with CPO ultimately enhanced pro-apoptotic activity. As determined in Chapter 5, there was an array of inflammation-activated pathways that may provide insight into the diverse symptomology observed with GWI.

\subsubsection{Experimental time points}

As timing is key in many studies, one limitation of these studies was the significant gap between time points ( 2 to $24 \mathrm{~h}$ ). Ideally, adding more time points would help provide additional information that leads to the apoptotic nature observed for both DFP alone and CORT+CPO, but accomplishing this can be difficult due to the large number of animals needed. Therefore, future studies must expand on this time course to further understand the progression of the phosphoprotein signaling. As this animal model also relates to the GWI disease state, it is crucial to investigate the permanency of these altered signal transduction pathways. Additional studies 
will also further probe these proteins and their post-translational modifications at later time points to better understand the long-term effects of these OPs.

\subsubsection{Total protein}

Another limitation of these studies was the lack of quantification of total protein expression of proteins that were measured for phosphorylation. Without total protein expression measurements of these phosphoproteins, it is unclear if the differences observed were due to changes in the underlying protein content or their specific phosphorylation states. Since the time course used in the present study is $24 \mathrm{~h}$ or less, it is expected that there would be little differences in total protein concentration. However, to further validate the findings observed, future studies would benefit from including a phosphoprotein to total ratio to help draw conclusions.

\subsubsection{Brain region}

The present studies examine several brain regions: the cortex and striatum postchlorpyrifos exposure, and the cortex and hippocampus post-diisopropyl fluorophosphate exposure. These regions were chosen in order to relate previous findings involving cytokine and acetylcholinesterase inhibition to the observed phosphoprotein signaling. The cortex plays a key role in attention, perception, thought, language and voluntary action, while the hippocampus plays a major role in learning and memory. The striatum is critical for the motor and reward systems (e.g., decision making, motivation, reinforcement, etc.). Therefore, all of these brain regions play into potential areas of focus for the symptoms associated with GWI. However, other regions of the central nervous system could be crucial to get a complete understanding of the brain response to these toxins. For example, future investigations of the cerebellum, which is involved in coordinating voluntary movements and motor skills (e.g., balance, coordination, and posture), 
could help link the phosphoprotein signaling responses to additional GWI symptomology. Additionally, while it is well established that the primary mode of action for these organophosphates is the brain, it may be beneficial to observe other major organs, such as the liver or kidneys, to determine the whole organismal response. 


\section{APPENDIX A: Chapter 5 Supplemental}

Table A.1. Proposed signaling nodes for a network generated by IPA at $0.5 \mathrm{~h}$ using the experimental dataset. The table represents the nodes that were found to be different in at least one exposure.

\begin{tabular}{|l|l|l|l|}
\hline DFP & CPO & CORT+DFP & CORT+CPO \\
\hline ARID5A & NCF4 & CARD6 & CARD6 \\
\hline CLEC6A & ARID5A & SH2D2A & LRBA \\
\hline HCK & CARD6 & TOLLIP & NCF4 \\
\hline NKX3-1 & HCK & PLCB3 & PIK3R2 \\
\hline CNR2 & MAP3K2 & MAP3K2 & CDH16 \\
\hline RASGRF1 & SH2D2A & NKX3-1 & CLEC6A \\
\hline LAT2 & TOLLIP & UBE2N & NKX3-1 \\
\hline NCF4 & CLEC6A & HCK & CNR2 \\
\hline PIK3R2 & LAT2 & OTUD7B & IQGAP1 \\
\hline
\end{tabular}

Table A.2. Proposed signaling nodes for a network generated by IPA at $2 \mathrm{~h}$ using the experimental dataset. The table represents the nodes that were found to be different in at least one exposure.

\begin{tabular}{|l|l|l|l|}
\hline DFP & CPO & CORT+DFP & CORT+CPO \\
\hline CD79B & CLEC6A & ADCY5 & AKT3 \\
\hline FCER1G & GPR19 & BTG1 & BTG1 \\
\hline LAT2 & IKBIP & HCK & CRK \\
\hline PLG & MAP3K4 & RASGRF2 & OTUD78 \\
\hline ARID5A & NQO2 & RPS6KA4 & RPS6KA3 \\
\hline CEACAM1 & RASGRF2 & GPR19 & TOLLIP \\
\hline FCGR2A & SH2D2A & PLCB3 & CARD6 \\
\hline LTB4R & TOLLIP & SH2D2A & NCF4 \\
\hline NCF4 & ATE1 & TNFRSF18 & RASGRF2 \\
\hline SELL & LAT2 & ATE1 & TRAFD1 \\
\hline TREM2 & TRAFD1 & CRK & ATE1 \\
\hline CLEC6A & CRK & PPP2R1A & CLEC6A \\
\hline FCGR2B & HCK & TRAFD1 & GPR19 \\
\hline HCK & PLCB3 & EGR4 & MAP3K2 \\
\hline RASGRF1 & UBE2N & HAVCR2 & PLCB3 \\
\hline TYROBP & CARD6 & MAP3K2 & UBE2N \\
\hline CD74 & MAP3K2 & NFKBID & NKX3-1 \\
\hline FGL2 & RPS6KA3 & RPS6KA3 & PPP2R1A \\
\hline
\end{tabular}


Table A.3. Proposed signaling nodes for a network generated by IPA at $24 \mathrm{~h}$ using the experimental dataset. The table represents the nodes that were found to be different in at least one exposure.

\begin{tabular}{|l|l|l|l|}
\hline DFP & CPO & CORT+DFP & CORT+CPO \\
\hline ARID5A & ALDH1L1 & KITLG & ALDH1L1 \\
\hline CEACAM1 & BTG1 & PIK3CB & CLEC6A \\
\hline FCGR2A & CRK & ROCK1 & GPR19 \\
\hline LTB4R & RASGRF1 & TSC1 & LAT2 \\
\hline NCF4 & RPS6KA3 & BRAF & NQO2 \\
\hline CLEC6A & ARID5A & Foxp1 & RASGRF2 \\
\hline FCGR2B & CD79B & IL7 & SH2D2A \\
\hline HCK & FCER1G & LRBA & ATE1 \\
\hline RASGRF1 & HCK & PIK3CD & TRAFD1 \\
\hline RPS6KA3 & RASGRF2 & TSC2 & CRK \\
\hline CD74 & SH2D2A & CDH16 & HCK \\
\hline FGR & TOLLIP & IQGAP1 & MAP3K2 \\
\hline RASGRF2 & ATE1 & NKX3-1 & NCF4 \\
\hline SELL & CLEC6A & PIK3R2 & PLCB3 \\
\hline TYROBP & FGR & VHL & UBE2N \\
\hline CD79B & PPP2R1A & HAVCR2 & CARD6 \\
\hline FCER1G & TRAFD1 & ITCH & FGR \\
\hline GPR19 & GPR19 & S1PR1 & MAP3K4 \\
\hline LAT2 & LAT2 & CNR2 & RPS6KA3 \\
\hline PLG & NCF4 & TNFSF10 & TIRAP \\
\hline
\end{tabular}

